University of Louisville

ThinkIR: The University of Louisville's Institutional Repository

Electronic Theses and Dissertations

$5-2014$

\title{
Functional implications of demyelination and the molecular control of remyelination in the adult mouse.
}

Nicholas John Kuypers

University of Louisville

Follow this and additional works at: https://ir.library.louisville.edu/etd

Part of the Anatomy Commons, and the Neuroscience and Neurobiology Commons

\section{Recommended Citation}

Kuypers, Nicholas John, "Functional implications of demyelination and the molecular control of remyelination in the adult mouse." (2014). Electronic Theses and Dissertations. Paper 786.

https://doi.org/10.18297/etd/786

This Doctoral Dissertation is brought to you for free and open access by ThinkIR: The University of Louisville's Institutional Repository. It has been accepted for inclusion in Electronic Theses and Dissertations by an authorized administrator of ThinkIR: The University of Louisville's Institutional Repository. This title appears here courtesy of the author, who has retained all other copyrights. For more information, please contact thinkir@louisville.edu. 


\title{
FUNCTIONAL IMPLICATIONS OF DEMYELINATION AND THE MOLECULAR CONTROL OF REMYELINATION IN THE ADULT MOUSE
}

\author{
By \\ Nicholas John Kuypers \\ B.A., Southern Illinois University at Carbondale, 1998 \\ M.S., University of Louisville, 2010

\begin{abstract}
A Dissertation
Submitted to the Faculty of the

University of Louisville School of Medicine

in Partial Fulfillment of the Requirements

for the Degree of:
\end{abstract}

Doctor of Philosophy

Department of Anatomical Sciences and Neurobiology

University of Louisville School of Medicine

Louisville, Kentucky

May 2014 

FUNCTIONAL IMPLICATIONS OF DEMYELINATION AND MOLECULAR CONTROL OF REMYELINATION IN THE ADULT MOUSE

By

Nicholas J Kuypers

B.A., Southern Illinois University Carbondale, 2008

M.S., University of Louisville School of Medicine, 2010

A Dissertation Approved on

January 24,2014

by the following Dissertation Committee:

Dissertation Director: Scott Whittemore, Ph.D.

Second Committee Member: David S. K. Magnuson, Ph.D.

Third Committee Member: Michal Hetman, Ph.D.

Fourth Committee Member: Meng Sheng Qiu, Ph.D.

Fifth Committee Member: Yong Li, Ph.D. 


\section{DEDICATION}

This dissertation is dedicated to:

Christopher Joseph Kuypers

My brother and childhood friend with whom not enough time was spent. 


\section{ACKNOWLEDGEMENTS}

I would first like to thank my dissertation advisor and mentor, Dr. Scott R. Whittemore, for his guidance, patience, support and friendship. I would like to that my committee member Dr. David S. K. Magnuson for his immeasurable contributions to my training. I would also like to thank my other committee members, Dr. Michal Hetman, Dr. Meng Sheng Qiu and Dr. Yong Li for their time, support and constructive input. I am very grateful to Christine Yarberry and Yi Ping Zhang for surgical support, Darlene Burke for statistical support, Kim Cash for animal care assistance and Johnny Morehouse, Will DeVries, Kurtis James, Minh Tran and Monica Vetter for assistance with behavioral and electrophysiological assessment. I am thankful to Kariena Andres for animal care support, Amberly Riegler and Ashley Mullins for husbandry and genotyping assistance, and Russ Howard, Jason Beare and Allison Metz for their cell culture support. I would like to show my appreciation to Toros Dincman, Scott Myers, Sujata Saraswat-Ohri and Amanda Pocratsky for their friendship, academic discourse and intellectual stimulation. Lastly, I am indebted to my wife, Mary Kuypers, for her love, sacrifice, endless support and inspiration. She is my driving force and without her, this dissertation would not be possible. 
TABLE OF CONTENTS

PAGE

COMMITTEE SIGNATURE PAGE

DEDICATION

ACKNOWLEDGEMENTS

ii.

TABLE OF CONTENTS

ABSTRACT

LIST OF FIGURES

iii.

iv.

V.

vii.

ix.

CHAPTER

1. INTRODUCTION 1

Preface 1

Oligodendrocyte Biology 1

Demyelination 9

$\begin{array}{ll}\text { Remyelination } & 19\end{array}$

MicroRNA (miRNA) Synthesis and Biological Function 27

miRNAs and Oligodendroglia $\quad 29$

Summary of Experimental Directions 30

2. FUNCTIONAL CONSEQUENCES OF ETHIDIUM BROMIDE DEMYELINATION OF THE MOUSE VENTRAL SPINAL CORD

Introduction $\quad 35$

Materials and Methods $\quad 36$

Results 44

$\begin{array}{ll}\text { Discussion } & 47\end{array}$

Acknowledgements 53 
3. CHARACTERIZING IN VIVO ELECTROPHYSIOLOGICAL ASSESSMENT IN THE ADULT MOUSE SPINAL CORD

Introduction

Materials and Methods

Results

Discussion

Acknowledgements

4. SFMBT2 CLUSTER MICRORNA (MIRNA) MIR-297C EXPRESSION INCREASES DURING MOUSE OLIGODENDROCYTE PROGENITOR (OP) DIFFERENTIATION AND REGULATES CELL CYCLE IN VITRO

Introduction

Materials and Methods

Results

Discussion

Acknowledgements

5. REGENERATIVE POTENTIAL OF THE CNS AND ITS CLINICAL LIMITATIONS

CNS Regeneration

The Modalities of Demyelination

Treating Demyelination Clinically

miRNAs as Therapeutic Targets: Prospective Treatment Strategies for Demyelination

miRNAs as Biomarkers of Demyelination 


\section{ABSTRACT \\ FUNCTIONAL IMPLICATIONS OF DEMYELINATION AND THE MOLECULAR CONTROL OF REMYELINATION IN THE ADULT MOUSE \\ Nicholas John Kuypers \\ January 24, 2014}

Oligodendrocyte $(\mathrm{OL})$ loss contributes to the functional deficits underlying diseases with a demyelinating component (Gajjar et al., 1997; Miller et al., 2002). Remyelination can restore these deficits (Duncan et al., 2009). Chapter 1 is an introduction to de- and re-myelination thus providing the necessary background information for consideration in subsequent chapters. Ethidium bromide (EB) has been extensively used in the rat and cat as a model of spinal cord demyelination. However, this lesion has not been addressed in the adult mouse. Therefore, Chapters 2 and 3 characterize a model of chronic EB-induced spinal cord demyelination in the mouse which can be assessed behaviorally and electrophysiologically.

MicroRNAs (miRNAs) possess both cell type- and differentiation stagespecific expression patterns (Lau et al., 2008). In oligodendrocytes (OLs), miRNAs regulate development and differentiation in vivo and in vitro, respectively (Lin et al., 2009; Zhao et al., 2010 and Dugas et al., 2010). However, it is unclear whether miRNAs involved in remyelination are distinct from those observed during normal myelination / development. Treatment of adult mice with the 
copper chelator cuprizone demyelinates specific brain regions which remyelinate following cuprizone cessation (Torkildsen et al., 2008). Therefore, Chapter 4 focuses on the role of oligodendroglial-specific miRNAs in cuprizone-induced deand re-myelination.

Lastly, Chapter 5 summarizes all of the data provided herein with an emphasis on clinical significance and therapeutic potential. 


\section{LIST OF FIGURES}

FIGURE

PAGE

1. miRNA-induced translational repression of target mRNA.

2. $\mathrm{IHC}$ and EC staining assess astrocytic reactivity and demyelination, respectively.

3. Functional and histological consequences of EB lesions.

4. Chronic inflammatory response following EB lesions.

5. Chronic axonal integrity following VWM EB lesions.

6. Neither spontaneous wheel running nor group housing improves hindlimb function following injections of $0.20 \mathrm{mg} / \mathrm{mL}$ EB.

7. Mouse tcMMEP response assessment.

8. Spinal cord laceration does not abolish subcortically-elicited MMEP responses.

9. Oligodendroglial CNPase-EGFP ${ }^{+}$reporter expression facilitates the quantification of cuprizone-induced de- and re-myelination.

10. FACS-based isolation of callosal CNPase-EGFP ${ }^{+}$cells yields a population cells with mRNA profiles highly consistent with OPs.

11. Transfection of $F A M^{\mathrm{TM}}$ Dye-labeled pre- or anti-miR scrambled RNA controls into MEFs for FACS-based 
12. Sorted CNPase-EGFP ${ }^{+}$populations do not contaminated by myeloid lineage cells.

13. Table of miRNAs identified in the microarray also consistent with oligodendroglial-derived or white matter-derived miRNAs previously identified in the literature.

14. Microarray data reveals that Sfmbt2 cluster miRNAs are significantly decreased at 6 weeks on cuprizone compared to no cuprizone miRNA expression levels.

15. Sfmbt2 cluster member mIR-297c displays a significant increase concomitant with differentiation in primary OPs.

16. miR-297c transfection regulates cell cycle progression in MEFs. 


\section{CHAPTER 1}

\section{INTRODUCTION}

Preface. Oligodendrocytes (OLs) are the sole source of myelin in the central nervous system (CNS). Their primary role is to form the myelin sheath, a dielectric structure composed of multiple tightly wrapped layers necessary for proper conduction of action potentials from the axon hillock to its terminus (Bradl and Lassmann, 2010). However, they also serve as a protective layer and provide trophic support to CNS axons (Nave, 2010). OLs promote optimal conduction velocity and intensity, both critical to maintaining efficient propagation of action potentials over long distances which can span up to several meters (Baumann and Pham-Dinh, 2001).

\section{Oligodendrocyte Biology}

A single OL extends multiple processes which can then myelinate tens to hundreds of axons. Therefore, within white matter structures (groups of highly myelinated axons), many OLs are present in a relatively even distribution along the axonal tract providing the myelin, protection and trophic support required for communication between grey matter regions (groups of neuronal cell bodies). 
$O L$ development. OLs are the last cells to develop in the CNS with the myelination of axons beginning during the second trimester in humans (Poduslo and Jang, 1984) and at postnatal day 0 in rodents (Semple et al., 2013). Myelination peaks soon after birth slowly tapering off throughout adolescence and adulthood with myelin levels beginning to decline at approximately age 40 in humans and 8-10 weeks in rodents (Bartzokis et al., 2010). During embryonic neural tube formation, oligodendrocyte progenitors (OPs) begin to arise primarily in the ventral spinal cord (Timsit et al., 1995) with a sub-population arising from the dorsal spinal cord (Qiang et al., 2010) as well as in the third ventricle (Gao and Miller, 2006) and begin to proliferate in high numbers. Using in vitro approaches, we now understand that newly generated OPs are generated under the mitogenic control of insulin-like growth factor 1 (IGF-1), fibroblast growth factor 2 (FGF2) and platelet derived growth factor alpha (PDGFa) secreted from neurons and astrocytes (Scolding et al., 1995; Frederick and Wood, 2004). This has been confirmed as intraventricular FGF2 administration in both pups and adult mice enhanced the production of OPs throughout the subventricular zone (SVZ) and within both the dorsal- (dSVZ) and inner-subventricular zones (ISVZ; Azim et al., 2012). They are identified in vivo by the immunohistochemical (IHC) and promoter based reporter expression of the transcription factor Olig2, the growth factor receptor platelet derived growth factor receptor alpha (PDGFRa), the proteoglycan NG2, 2',3' cyclic nucleotide phosphodiesterase (CNPase) and the surface ganglioside A2B5 (Baumann and Pham-Dinh, 2001). Moreover, the expression of each of these OP-specific markers has also been demonstrated in 
vitro. Once formed, newly generated OPs migrate throughout CNS white matter directed by $\mathrm{N}$-methyl-d-aspartic acid (NMDA) receptor (NMDAR) signaling (Xiao et al., 2013). Migration ceases for spinal-derived OPs when they terminate at an axonal segment lacking myelin within the brain or spinal cord. OPs which arise from the $3^{\text {rd }}$ ventricle follow a separate migratory route ultimately terminating at and myelinating the optic nerve (Ono et al., 1997). The ventral OP differentiation signaling pathway is sonic hedgehog (Shh) dependent while the dorsal pathway is not (Mitew et al., 2013; Ortega et al., 2013). Moreover, not all OPs form oligodendrocytes as some form astrocytes which occurs primarily in grey matter (Temple and Raff, 1985) while a small subset of OPs do not differentiate during normal development and remain evenly distributed throughout the grey and white matter in an immature mitotically competent state (Gensert and Goldman, 1997). The role of OPs which persist throughout adulthood is addressed in more detail in Chapter 1, Remyelination. Once at their terminal location, myelinating OPs begin to mature into pre-myelinating OLs which express differentiation markers galactosylceramidase (GalC) and the sulfatides $\mathrm{O} 4$ and $\mathrm{O} 1$ both in vivo and in vitro (Levine et al., 2001). Differentiation into myelinating OLs has been described in vitro to be under the transcriptional control of Olig2, Olig1, Nkx2.2 (Joubert et al., 2010) and ying yang 1 (YY1; He and Casaccia-Bonnefil, 2008) as each of these studies co-localized expression of oligodendroglial specific markers with transcription factor expression. As a last step in myelination, the myelin compaction proteins proteolipid protein (PLP), myelin basic protein (MBP), myelin associated glycoprotein (MAG), myelin oligodendrocyte glycoprotein 
(MOG) and CNPase begin to increase their expression (Huang et al., 2013). This has also been confirmed in vitro as myelin compaction protein expression and mRNA levels increase concomitant with differentiation even in the absence of axons (Armstrong et al., 1990; Joubert et al., 2010).

Myelin composition. Mature myelin consists of tightly wrapped layers of lipid membrane densely integrated with myelin compaction proteins PLP, MBP and to a lesser extent MAG, MOG and CNPase (Morell and Quarles, 1999). The regulation and maintenance of myelin is a dynamic process as even small disruptions in mature myelin proteins can result in substantial axonal dysfunction (lester et al., 1997). It has been suggested that optimal myelination levels can be promoted by skill training and physical activity (Sampaio-Baptista et al., 2013) and by maintaining a healthy diet (Tomasch, 1961).

Biomechanics of myelin sheath formation. When the elongating process of a maturing $\mathrm{OL}$ reaches an unmyelinated axon, a process unfolds beginning with rapid cell adhesion molecule-induced myelin protein synthesis, axon wrapping, compaction and finally termination of the myelination process. Initially, integrin receptors on the developing OL processes are activated by axonal integrin receptors when the axoglial connection is formed resulting in the release and translation of pre-transcribed MBP mRNA from RNA binding proteins at the site of myelination, distal to the OL soma thus explaining how myelin protein synthesis occurs so soon after axonal contact (Laursen et al., 2011). The newly 
formed axoglial association is further strengthened by local release of both glutamate and action potential-induced adenosine triphosphate (ATP) release which is recognized by NMDA receptors and purinergic receptors on the OL, respectively (Matute, 2011; Wake et al., 2011). Both cell adhesion molecule- and glutamate/ATP-induced molecular cascades converge on the Fyn kinase, increasing its activity thus driving OL process elongation (Klein et al., 2002).

The process of myelin wrapping remains incompletely elucidated in the CNS. However, in the peripheral nervous system (PNS), Schwann cell myelin wrapping has been described in much more detail. From a molecular standpoint, the wrapping of peripheral axons by Schwann cell myelin requires the signal transduction protein neural Wiskott-Aldrich syndrome protein (N-WASP) which interacts with activated cell surface receptors ultimately driving the cytoskeletal actin polymerization necessary for membrane elongation and myelination (Novak et al., 2011). In genetically engineered mice lacking N-WASP, ultrastructural analysis revealed that peripheral myelin was morphological abnormal with few instances of tightly wound myelin arranged in uniform layers (Novak et al., 2011). What is understood about OL myelin wrapping is that lamellipodia formation occurs resembling Schwann cell myelination. Moreover, this process is driven by actin polymerization and is mediated by Wiskott-Aldrich syndrome protein family verprolin homologous (WAVE) proteins during myelination (Bauer et al., 2009). Myelin wrapping must occur until the appropriate thickness of myelin is attained with the optimal myelin sheath thickness being directly proportional to the 
diameter of an axon (i.e. larger diameter axons require thicker myelin sheaths) and this proportional relationship has been termed the G-ration (Franklin and ffrench-Constant, 2008). G-ratios allow for experimental quantification of myelin status and thickness and can be a useful diagnostic tool to assess the overall functional capacity of white matter.

Following myelin wrapping is myelin compaction which involves certain proteins and glycoproteins present in the myelin sheath but this process is still not completely understood. However, it is clear that not all mature myelin proteins are necessary for compaction to occur. PLP null mutants still display myelin compaction (Bakhti et al., 2013) while MBP null mutants exhibit a clear loss of compact myelin resulting in substantial dysfunction (Readhead and Hood, 1990). There is a loss of the negatively charged oligosaccharide-induced electrostatic repulsion which normally prevents bilayer-bilayer interaction. This allows for multiple layers of OL process lipid bilayers to come into close enough proximity with adjacent bilayers to allow myelin protein interactions/binding to occur (Bakhti et al., 2013).

Myelin internode formation and saltatory conduction. The presence of a myelin sheath was first described by the German pathologist and anatomist Rudolf Virchow (Virchow, 1854). A decade later, the French pathologist Louis-Antoine Ranvier identified gaps in the myelin sheath initially described by Virchow and termed the structures nodes (gaps) of Ranvier. Now, more than a century 
following Virchow and Ranvier's discovery of intermodal myelin, we currently understand that internodal myelin formation on axons is accompanied by changes in axonal membrane ion channel distribution which is crucial to the proper conduction of action potentials along the axon. Normally, an unmyelinated axon has voltage gated sodium and potassium channels, $\mathrm{Na}^{+} / \mathrm{K}^{+}$ATPases and $\mathrm{Na}^{+} / \mathrm{Ca}^{2+}$ exchangers evenly distributed along its membrane (England et al., 1990). As the axoglial association forms and OLs begin to wrap an axon with segments of myelin, the ion channels redistribute within the axonal membrane clustering at the nodes of Ranvier (Black et al., 1990).

Not all axons are myelinated. The small diameter unmyelinated A-delta and Cfibers within ventral spinal white matter that convey pain/temperature sensory information do not myelinate. These fibers conduct action potentials at a much slower rate than do larger diameter myelinated fibers (Sotnikov, 2003).

Therefore, myelination seems to be crucial to action potential conduction velocity which becomes increasingly important when considering that some large diameter efferent axons must travel several meters before terminating on a lower motor neuron in the anterior grey horn of the spinal cord. Once an axon has been properly myelinated with compact layers of myelin at the appropriate G-ratio, internode formation has occurred and ion channels have clustered at the nodes of Ranvier, rapid and efficient conduction of action potentials by myelinated fibers termed saltatory conduction can now take place. Saltatory conduction prevents the decay of action potential intensity as action potentials jump from node to 
node triggering action potentials at each node of Ranvier as it travels down the myelinated axon thus promoting consistent and rapid action potential propagation (Tasaki, 1939).

Metabolic and trophic support of axons by OLs. Aside from the primary role for OLs in saltatory conduction, myelinating OLs also provide metabolic and trophic support to axons. Lee et al. (2012) recently demonstrated that the lactate shuttling monocarboxylate transporter 1 (MCT1) is highly enriched in OLs and disrupting oligodendroglial MCT1 results in both axon damage and neuronal loss in experimental models. Moreover, reduced MCT1 protein expression is a hallmark pathology observed in patients and in experimental models amyotrophic lateral sclerosis (ALS) which has provided a new glial based mechanism for ALS, a disease not classically thought of as involving OLs (Lee et al., 2012).

Lastly, trophic support is provided to axons by myelinating OLs via secretion of insulin-like growth factor (IGF-1) and glial derived neurotrophic factor (GDNF). IGF-1 has been demonstrated to promote cortical neuron survival (Wilkins et al., 2001). Both OPCs and OLs secrete factors which promote local axon viability via a PI3 kinase/Akt-signaling pathway. On the other hand, only mature OLs secreted factors which resulted in enhanced neurofilament phosphorylationdriven neurite outgrowth via a MAP kinase/Erk-dependent signaling pathway. Oligodendroglial GDNF has been identified as a primary factor responsible for the MAPK/Erk-driven axonal outgrowth and together with IGF-1, provides an 
axonal microenvironment in favor of axon survival and outgrowth (Wilkins et al., 2003).

\section{Demyelination}

Following multiple CNS diseases, ischemia and trauma, OL loss (demyelination) is typically observed to varying degrees within white matter. The degree of demyelination is dependent on the nature and severity of the disorder which will be more specifically addressed below. However, there is a biological sequelae associated with demyelination, and with the loss of protection and loss of trophic support to axons.

Specifically, complete OL loss, perturbed OL maintenance and lack of myelin compaction each can result in dysfunctional axonal conduction. Therefore, conventional imaging techniques such as diffusor tensor imaging (DTI) or computed tomography (CT) scans, while adequate at identifying widespread OL loss in white matter, may not be sensitive enough to accurately identify subtle changes in myelin compaction and loss of trophic support. However, in each of these cases, axonal conduction properties will be compromised.

Aside from the problems associated with altered or abolished electrical conduction, the lack of protection provided by OLs leaves axons susceptible to a host of potential secondary insults, including but not limited to: glutamate toxicity, 
$\mathrm{Ca}^{2+}$ influx, systemic inflammatory cell infiltrate and reactive oxygen species (ROS) production. Each of the events mentioned are associated with the loss of axonal protection and have been implicated in axonal damage or neuronal death. Moreover, OL loss also results in a loss of trophic support normally provided by mature OLs to axons. As a result, focal CNS demyelination can have profound effects on the survivability of demyelinated axons not only within the demyelinating lesion (as identified via MRI imaging), but in more remote areas of white matter where myelin trophic support and myelin compaction are suboptimal following the secondary demyelination which is not identifiable. Without the OL-provided trophic support, axons will begin to degenerate in regions of severe OL loss, but this effect is not limited to only the demyelinated areas as the trophic factors secreted by OLs also contribute to axonal viability in white matter areas remote to the lesion. Taken together, this suggests that although demyelination is primarily due to a loss of myelinating OLs, there are also secondary components involving neurons and other glia as well.

\section{Clinical Significance of OL loss}

Significant functional loss is observed in multiple post-traumatic induced- and disease-induced forms of demyelination. Although the variety of sub-classes within each of these diseases is vast, they can be classified into 2 main groups: Genetic demyelinating disease and acquired demyelinating disease (Ben-Hur and Goldman, 2008). Each of these present a substantial reduced patient quality 
of life coupled with a long-term financial burden. As each group of demyelinating disease is unique biologically, clinically and etiologically, they will be independently addressed in detail below.

1. Genetic demyelinating diseases. The leukodystrophies comprise a collection of disorders resulting in the loss or dysfunction of not only OLs, but other glia and neurons in both children and adults (Kohlschütter and Eichler, 2011). Although the primary anatomical correlate of dysfunction associated with each of these is OL loss, the nature of this loss stems from heterogeneous causes which are addressed in more detail below.

Pediatric Leukodystrophies (Alexander disease, Canavan disease and Krabbe disease) are a group of rare inherited disorders resulting in fatality during childhood. Limited treatment options are available but even in the best cases, mortality occurs prior to adolescence. Canavan disease is the most common pediatric leukodystrophy with an onset typically observed at birth with fatality occurring before 4 years of age (Matalon and MichalsMatalon, 1999). However, in patients with milder forms of the disease, life expectancy can extend into the early twenties (Gordon, 2001). It is an autosomal recessive disease caused by a mutation in the aspartoacylase (ASPA) gene resulting in deficient enzymatic breakdown of $\mathrm{N}$-acetyl aspartate. When not properly metabolized, NAA accumulates resulting in a chemical imbalance which interferes with myelin formation during development. Moreover, NAA accumulation is also toxic to existing myelin 
(Matalon \& Michals-Matalon, 1999). Assadi et al. (2010) recently demonstrated that treatment with lithium citrate slightly reduced intracerebral NAA levels in a cohort of 6 infants $<10$ months old. However, no treatment effect was reported for clinical outcome and myelin status.

Alexander disease typically occurs from birth to 2 years of age (Alexander, 1949; Messing et al., 2012). However, in very rare cases, a juvenile form (onset by 6 years of age) and an adult form (onset by 10 years of age) can be observed (Pareyson et al., 2012; Messing et al., 2012). It is an autosomal dominant disease caused by mutation in the GFAP gene and primarily affects white matter in the brain stem and cerebellum (Li et al., 2002). Krabbe disease typically occurs from birth with patient's life expectancy being less than 2 years of age due to its rapid progression (Kohlschütter and Eichler, 2011). However, rare cases of a late onset form have been documented and normally display a slower progression (Debs et al., 2013). It is an autosomal recessive disease resulting from mutations in the GalC gene located on chromosome 14 (Sakai, 2009). GalC is responsible for the breakdown of sphingolipids, a major component of myelin following GalC metabolic dysfunction, resulting in lipid accumulation which interferes with proper myelin formation and compaction (Sakai, 2009). 
Vanishing white matter (VWM) disease is unique among the leukodystrophies as it seems to only affect OLs and astrocytes with little to no pathology seen in other CNS glia, vasculature or axons (van der Knaap et al., 2006). Onset is thought to occur at birth with patients first being symptomatic between 2-6 years of age (van der Knaap et al., 2006). However, late onset has been documented late into adulthood and is commonly misdiagnosed as schizophrenia due to white matter lossinduced psychosis (Matsukawa et al., 2011; La Piana et al., 2012). Although it has been characterized as an autosomal recessive disease caused by a mutation in any one of the 5 genes which encode various subunits of the translation initiation factor EIF-2B (EIF2B1, EIF2B2, EIF2B3, EIF2B4, and EIF2B5; Pronk et al., 2006), the exact mechanism of OL death remains elusive. One theory of mechanism is that OLs and astrocytes both synthesize large amounts of proteins putting OLs under high metabolic demand. Therefore, when production of translation initiation factors are perturbed, the metabolic demand becomes too exhaustive for the OL to maintain and overall protein synthesis becomes dysfunctional or completely compromised depending on which specific subunits are affected by the mutation. OL death imminently proceeds as apoptotic cascades are initiated following the loss of metabolic sustenance (Pronk et al., 2006). Following clinical diagnosis, a myriad of progressive symptomology is observed including coma, followed by death. 
Lysosomal storage disorders are diseases characterized by dysfunctional lipid metabolism. Metachromatic leukodystrophy (MLD) is a common form of lysosomal storage disease and presents in 3 forms: late infantile, juvenile and adult. Late infantile MLD is the most common and children usually die before the age of 5 (Fluharty, 2006). It is an autosomal recessive disease classified by an arylsulfatase A deficiency resulting in sulfatide accumulation-induced toxicity which is well accepted as being responsible for the loss of myelin (Poeppel et al., 2005). However, sulfatide accumulation has been suggested by others to be non-toxic bringing into question classical dogma (Blomqvist et al., 2011). These authors have proposed a model where sulfatide accumulation is not the sole source of demyelination as lysosphingolipid accumulation (a metabolite of sulfatide) may also play a role as it is cytotoxic to OLs in vitro (Blomqvist et al., 2011). The late infantile form typically presents clinically around 4-8 months as severe motor and developmental dysfunction with few children surviving past the age of 5 (Kohlschutter and Eichler, 2011). The juvenile form can present as early as age 3 but more commonly is recognized when the patient begins to display progressive cognitive dysfunction in school eventually resembling infantile MLD. These patients normally die within 10-15 year of diagnosis (Krageloh-Mann et al., 2013). The adult form begins in late adolescence initially presenting as a psychiatric disease which slowly progresses to dementia and ultimately 
death. However, adult onset MLD is the slowest progressing form of MLD with patients having reported living for decades (Sadovnick et al., 1993).

2. Acquired demyelinating diseases. Acquired demyelinating diseases comprise a collection of disorders primarily affecting adults but in rare occasions may affect the pediatric population as well.

Multiple sclerosis (MS), the leading cause of neurological disease in young adults, is the most common of disease in this group (Evans et al., 2013). It was initially suggested to be an autoimmune disease as there are similarities between T-cell immunoreactivity in human MS and experimental allergic encephalomyelitis, a rodent model of MS (Constantinescu et al., 2011). However, others have countered that demyelination is not induced following transfer of serum or T-cells from an MS patient (Jones et al., 1995). Its hallmark pathology is the presentation of MS plaques throughout the white matter and can be identified with conventional magnetic resonance imaging (Ge, 2006). Although MS etiology has been suggested to involve both environmental factors and genetic susceptibilities, identification of the specific biological events that result in acute inflammatory injury to axons and glial cells remains elusive. MS can further be categorized into 4 sub-groups: Classic MS, acute MS, neuromyelitis optica and concentric sclerosis. Although each sub-group displays the hallmark characteristics of MS, a brief description of their 
distinctions in provided below. Acute MS is difficult to categorize as it has been suggested to be very aggressive forms of acute-disseminated encephalomyelitis (ADEM), an inflammatory disease primarily affecting children (Bester et al., 2014). In any case, its clinical presentation is rare. Neuromyelitis optica is distinct from classical MS in that during the acute phase, clinical presentation starts with vision loss (due to optic nerve demyelination) and soon progresses to paraplegia and sensory loss (due to spinal cord demyelination). Patients who survive the acute phase normally have chronic functional deficits resulting from demyelination and axonal loss within the spinal cord. Lastly, concentric sclerosis is perhaps the most unmistakable form of multiple sclerosis with a clinical presentation characterized by bands of white matter lesions which alternate with bands of normal appearing white matter. It typically progresses rapidly and may also occur concomitantly with more classical MS plaques (Sato et al., 2012).

Ischemic-induced demyelination is most commonly observed in cerebrovascular disease, with the primary pathology located in subcortical white matter. These patients will develop dementia and neurological deficits with the most identifiable characteristic being marked arteriosclerosis and arteriolosclerosis within the deep cerebral white matter, typified by reactive gliosis and cavitation (Kelley, 2006). 
Traumatic brain injury (TBI) and spinal cord injury (SCI) comprise the most complex diseases which include a demyelinating component. Although both $\mathrm{TBI}$ and $\mathrm{SCl}$ are implicated as primary and secondary inducers of demyelination, more attention has focused on $\mathrm{SCl}$ in the context of demyelination because it has a much higher proportion of myelin relative to the brain. Injury spinal white matter and the subsequent demyelination is well described (Norenberg et al., 2011). Not only is there a component of vascular disruption similar to what is observed following stroke, but SCI can involve direct damage to all cell types local to the lesion as well as chronic secondary effects in spinal segments distant to the lesion epicenter. In animal models, chronic demyelination has been described (Gledhill et al., 1973; Blakemore, 1974; Gledhill and McDonald, 1977; Blight, 1983; Totoiu and Keirstead, 2005) while little chronic demyelination is reported in humans (Gensert and Goldman, 1997; Kakulas, 1999; Guest et al., 2005). However, recent evidence has suggested that remyelination is far more extensive in animal models when accounting for which axons remain spared and traverse the lesion (Lasiene et al., 2008).

Remyelination occurred successfully when axon viability was maintained making the extent of remyelination directly proportional to the number of spared axons. This agrees with clinical data suggesting that successful spontaneous remyelination can also occur in human $\mathrm{SCl}$ but following chronic demyelination, axons begin to degenerate and neurons die limiting the capacity for remyelination. Considering that remyelination is slow, it is 
imminent that even in the best case scenarios, at least some axons will be lost. Moreover, experimental advances made in the field of axonal regeneration have now demonstrated that spinal cord axons can regrow over long distances (Houle and Cote, 2013) but these newly formed axons would not have myelin making their functional capacity questionable. Therefore, any therapeutic strategy attempting to promote the regrowth of axons across a lesion would certainly require supplementation of available OP pools with transplanted OPs in order to provide the glial support required for optimal axonal conduction. Taken together, these considerations suggest that increasing the overall numbers of endogenous OPs or supplementing OP populations with transplantation approaches both have the potential to enhance remyelination and reduce axon loss if applied before demyelination reaches a chronic stage or following axonal regrowth.

Other etiologies included in acquired demyelinating disease are chemicaland viral induced demyelination, which are both rare with few common mechanism of induction making them more difficult to define as a thematic group and they will not be addressed here. 


\section{Remyelination}

Remyelination was first described in the PNS following crush-induced demyelination of the sural nerve in rabbits (Quilliam, 1958). It was first described in the CNS following demyelination of feline spinal cord by repeatedly withdrawal and re-injection of cerebrospinal fluid (CSF; Bunge et al., 1961) and since has also been extensively described in rodents (Blakemore and Franklin, 2008), primates (Radtke et al., 2004) and humans (Kipp et al., 2012). In rats, toxic demyelination models using ethidium bromide (EB; Blakemore and Franklin; 2008) and lysolecithin (Gregson and Hall, 1973) as well as trauma-induced demyelination (SCl; Totoiu and Keirstead, 2005) have provided a platform to assess remyelination which takes advantage of the advanced behavioral assessment methods available for rat studies (Onifer et al., 2007). In mice, cuprizone ingestion (Torkildsen et al., 2008), experimental autoimmune encephalopathy (EAE; Constantinescu et al., 2011) and mutant strains of mice lacking in genes necessary for myelin formation (Windrem et al., 2008; Wang et

al., 2013) have all been used as a platform to assess remyelination. Of all rodent demyelination models, low concentration EB (Franklin and ffrench-Constant, 2008) and lysolecithin (Jeffery and Blakemore, 1995) injection as well as the cessation of cuprizone demyelination (Matsushima and Morell, 2001) each exhibit a spontaneous remyelination response which is driven by OPs. Work done employing these models has been used to generate data which has 
provided much of our knowledge about the signaling pathways and mechanisms involved during remyelination.

Oligodendrocyte progenitors (OPs). It has been well described that OPs persist in adult rodent white matter in a mitotically competent state and are the primary substrate by which endogenous remyelination occurs (Blakemore et al., 1995; Gensert and Goldman, 1997). OPs have also been isolated from adult human white matter using FACS based identification of reporter expression driven off of OP-specific promoters (Roy et al., 1999). When cultured, the human OPs (hOPs) proliferated and differentiated into mature mixed glial populations with a predominantly oligodendroglial phenotype. It has been suggested that successful endogenous remyelination in the CNS is often perturbed due to axon loss, inflammation, myelin debris, the glial scar and lack of the appropriate cues required to direct remyelination (Back et al., 2005; Kotter et al., 2006; Fuller et al., 2007; Giger et al., 2008; Fancy et al., 2009). However, this has been challenged as being an animal model-specific phenomenon as spontaneous remyelination has been described in human MS patients (Kipp et al., 2012). This discrepancy has been suggested to result from a failure to assess remyelination of intact axons as accounting for this discrepancy showed near normal myelination patterns in spared rodent axons following spinal contusion (Lasiene et al., 2008). 
OP engraftment. In an effort to enhance endogenous remyelination, OP cell transplantation studies in animals have begun to identify the necessary considerations for implementation in the clinical setting. Many groups have demonstrated that following transplantation of OPs isolated in vivo, there is evidence of graft survival and successful integration within the host CNS. However, obtaining OPs with approaches that isolate them from whole tissue does not yield the number of OPs necessary for clinical consideration (Ben-Hur and Goldman, 2008). Therefore, several groups have begun to use embryonic stem (ES) cells as a source for OPs as they can be limitlessly expanded ex vivo prior to their differentiation into OPs (Ben-Hur and Goldman, 2008). Brustle et al. (1999) was the first to report remyelination following ES cell-derived OP engraftment. Using a rat model of Pelizaeus-Merzbacher disease (PMD) where (similar to human patients) PMD rats carry a mutation in the X-linked PLP gene resulting in myelin deficiency (Hodes et al., 1993), isolated ES cells were differentiated in vitro into OPs, expanded to scale for transplantation and injected into the spinal cord of PMD rat pups (Brustle et al., 1999). Two weeks postinjection, ultrastructural analysis of demyelinated white matter within the dorsal column of OP-injected PMD rats exhibited abundant myelin sheaths as compared to control PMD rats. Han et al. (2004) isolated $\mathrm{A}^{\mathrm{B}} \mathrm{B}^{+}$glial restricted precursor (GRP) cells from E13.5 rat embryos transgenically expressing alkaline phosphatase using flow activated cell sorting (FACS). Sorted GRPs were then expanded and engrafted into the cervical spinal cord following unilateral hemisection of the lateral funiculus. Tracking the GRP grafts with human alkaline 
phosphatase expression revealed that they migrated throughout the lesion and matured in a context specific manner (i.e. astrocytic phenotype in grey matter and oligodendroglial phenotype in white matter) but axonal remyelination, functional recovery and the efficiency of OL genesis was not reported. This may be due to the author's choice of lateral funiculus laceration as an injury model which would presumably spare few axons leaving behind a poor substrate for remyelination. However, when engrafted into the adult dysmyelinating shiverer mouse, a model with spared axons but lacking compact myelin, transplanted human ES cell-derived OPs were able to integrate, differentiate and form compact myelin (Nistor et al., 2005). Of all transplantation studies mentioned, most do not report behavioral function or electrophysiological responses, diagnostics necessary to assessing the integrity of functional myelin. This may be because the model used to assess demyelination did not provide clear-cut outcome measures sufficient for a functional deficit to be accurately assessed. Therefore, because of the variety of behavioral and electrophysiological tools available for functional assessment following $\mathrm{SCl}$, the spinal cord has provided a useful substrate to assess functional remyelination. Specifically, the Basso, Beattie and Bresnahan (BBB) scale for open-field locomotor function (Basso et al., 1995) and trans-cranial magnetic motor-evoked (tcMMEP) response conduction for electrophysiological function (Magnuson et al., 1999) are the most common techniques used to evaluate behavioral function and electrophysiological response conduction, respectively. Therefore, several studies have investigated the therapeutic potential of OP engraftment using $\mathrm{SCI}$ 
as a model for demyelination (Bambadakis and Miller, 2004; Cao et al., 2005; Karimi-Abdolrezaee et al., 2006; Cao et al., 2010; Hofstetter et al., 2005). In all but one publication (Kierstead et al., 2005), the transplanted OPs had been engineered to express growth factors. However, not only were the growth factors manipulated different, but the source of OP was different in each experiment: Bambadakis and Miller, 2004 - OPs immunopanned from rat pup cortex / Shh; Cao et al., 2005 - glial restricted precursors (GRPs) / Brain derived neurotrophic factor (BDNF)/Neurotrophin-3 (NT3); Karimi-Abdolrezaee et al., 2006 - Neural precursor cells (NPCs) / Platelette derived growth factor (PDGF), fibroblast growth factor 2 (FGF2) and endothelial growth factor (EGF); Cao et al., 2010 OPs ilsolated from adult rat spinal cord / ciliary neurotrophic factor (CNTF); Hofstetter et al., 2005 - NPCs / Neurogenin-2). Unfortunately, there is a significant lack of standardization across laboratories with respect to therapeutic manipulation, choice of modelling and choice of cell source. This is certainly an issue which must be reconciled in order to effectively and reliably replicate experimental data with the hope to ultimately translate these therapies to the clinic.

The utility of using rodent models to address questions specific to human oligodendrocyte biology has been useful. However, the experimental advantage of using human- rather than animal-derived OPs could obviate any concerns of species related differences between experimental animals and human patients as a limitation to translating research from bench to bedside. To that end, 
chimeric mouse models were developed as a tool to assess hOP-driven remyelination in vivo thus providing insight into the source-dependent phenotypic differences in hOPs isolated from adult or fetal tissue as well as the feasibility of hOP engraftment as a clinically relevant treatment (Windrem et al., 2004).

Human OPs can be isolated from both tissue derived sources (adult subcortical white matter or fetal forebrain) via FACS-based cell surface antigen detection or promoter-based reporter expression (Roy et al., 1999; Windrem et al., 2004) as well as derived from stem cell-like progenitor sources (embryonic or induced pluripotent stem cells hitherto referred to as hES cells and hiPSCs, respectively (Roy et al., 1999; Windrem et al., 2004). The engrafted fetal-derived hOPs displayed robust migratory behavior and differentiated in a context specific manner (i.e. OL differentiation in white matter and astrocyte differentiation in gray matter). However, their purity was marginal and the rate at which the remyelination occurred was slow. On the other hand, engrafted adult-derived hOPs were highly oligodendrocytic and remyelinated at a much faster pace than their fetal-derived counterparts but they displayed limited migratory distances and lacked the context specificity observed in transplanted fetal-derived hOPs when fully incorporated into the mouse CNS. Each hOP source has its advantages which may be exploited to adapt as treatments for different demyelinating diseases. For example, the widespread migratory patterns and context specific differentiation of fetal-derived hOPs may be more effective at treating the congenital leukodystrophies or the enzymatic pediatric leukodystrophies whereas 
the high purity and rapid rate of remyelination in adult-derived hOPs may be more suitable for treating ischemic demyelination (as in stroke) or postinflammatory demyelination (as in multiple sclerosis). However, in both cases, challenges persist as allograft rejection is an ongoing concern. Moreover, limited tissue-derived hOP yield suggests that the idea of relying on isolated hOPs as a graft source scalable for clinical use may be unrealistic. Several groups have investigated the possibility of using human embryonic stem (hES) cells as a renewable source of hOPs but this too presents its obstacles (Nistor et al., 2005; Kang et al., 2007). Limited understanding of hES cell differentiation protocols and a lack of technology to absolutely exclude all potentially tumorigenic hES and hES-like cells from mixed cultures combined with allograft rejection concerns excludes hES cell-sources as a viable means to generate hOPs in high yield and purity. Moreover, although many genetic similarities are shared between hES cell-derived and fetal tissue derived neural progenitors, there are disparate differences observed when attempting to differentiate these progenitors into dopaminergic neurons as $\mathrm{hES}$ cell-derived progenitors could be driven to this phenotype while fetal-derived could not (Shin et al., 2007). It is likely that similar differences will be observed between differentiating hES cell-derived and fetal tissue-derived hOPs.

Focus has recently shifted away from acquiring hOPs from hES cell sources with a particular interest in acquiring hOPs from human induced pluripotent stem cell (hiPSC) sources, primarily in an attempt to obviate immunorejection concerns. 
hiPSCs engineered from adult fibroblasts can be indefinitely expanded in culture and differentiated into a myriad of CNS cell types both in vitro and in vivo (Yamanaka and Takahashi, 2006; Almeida et al., 2012; Emdad et al., 2012; Wang et al., 2013). Specifically, hiPSC-derived hOPs (hiPSC-OPs) have been recently shown to rescue the congenital hypomyelinating mouse (shiverer) using similar techniques to work previously mentioned with isolated fetal- and adultderived hOPs (Wang et al., 2013). The hiPSC-OPs displayed migratory activity and efficiency phenotypic of fetal-derived hOPs following neonatal chimerization of the shiverer mouse CNS. Moreover, long term monitoring following hiPSC-OP engraftment revealed no evidence of teratoma formation in all transplanted mice suggesting that hiPSC-OPs are non-tumorigenic in vivo (Wang et al., 2013). Taken together, these data suggest that as hiPSC-OPs are fit for scalable expansion, have a reduced concern for rejection and display no tumorigenicity, they carry the most potential for clinical translation when compared to hES cellderived or tissue-derived hOP sources.

A caveat to the OP transplantation studies performed in the shiverer mouse is that although the shiverer mouse provides a useful model of genetic pediatric demyelination as affected mice typically die by one month (Chernoff, 1981), it is far more permissible to remyelination that the complex and hostile microenvironment observed following $\mathrm{SCl}$ or repeated episodes of inflammatory demyelination. Thus, while the shiverer mouse may provide a tool to assess OP 
graft myelination potential, its utility as a model to assess OP engraftment from a translational perspective may be limited.

\section{MicroRNA (miRNA) Synthesis and Biological Function}

MicroRNAs (miRNAs) are a family of evolutionarily conserved 22-24 nucleotide noncoding RNAs regulating protein translation via posttranscriptional gene repression (Ambrose, 2004) thus acting as fine-tuners of eukaryotic gene expression. Recent literature supporting this hypothesis demonstrated a modest rather than an absolute suppression of protein output by miRNAs (Baek et al., 2008; Selback et al., 2008). However, this does not obviate the need for research involving miRNAs as therapeutic targets as there several examples where a small fold change in miRNA expression levels results in dramatic alterations of cellular processes; this may be due to miRNAs ability to repress many targets with similar functions (Flynt and Lai, 2008). miRNA genes are primarily encoded within the introns of protein-coding genes, and to a lesser extent in monocystronic units and polycystronic clusters (Bartel, 2004). RNA polymerase II is responsible for the transcription of miRNA genes into stem-loop transcripts termed primary miRNAs (pri-miRNAs). Following cleavage by the RNase III nuclease Drosha, pri-miRNAs yield a $\sim 65 \mathrm{nt}$ precursor miRNA (pre-miRNA) which is exported out of the nucleus by Exportin 5. Once in the cytoplasm, premiRNAs are processed by Dicer, a second RNase III enzyme yielding 19-25 double stranded duplexes which are subsequently unwound into sense and 
antisense strands by a helicase. The mature miRNA strand can then associate with Argonaut proteins and other factors to form the miRNA-induced silencing complex (miRISC; Fig. 1A,B). Argonaute proteins provide the catalytic activity of the miRISC while the miRNA drives the targeting of the miRISC (Cenik \& Zamore, 2011). The ability of miRNAs to target certain mRNAs primarily relies on a miRNA "seed" region (nucleotides 2-9 at the 5' end of the miRNA). When targeting exhibits a true complementarity (Carthew and Sontheimer, 2009), mRNA degradation is favored. When targeting is imperfectly complimentary, translational repression is favored (Hu and Coller, 2012). Association of the miRISC with a target transcript finally results in a reduction in protein levels for target transcripts whether it occurs via translational repression or mRNA degradation (Baek et al., 2008; Selback et al., 2008).

It is the nature of this imperfect complimentary binding that lends to the potential of using and/or targeting miRNAs as therapeutic targets for disease. Considering that miRNA binding is primarily directed by base pairing of the 7-8 nucleotide seed sequence to target mRNA rather than base pairing of the entire 22-23 nucleotide sequence to target mRNAs in perfect complementarity, the wide range of mRNA targets which can be regulated by one miRNA can be in the hundreds to the tens of thousands (Ghelani et al., 2012; John et al., 2004). Therefore, therapeutic manipulation of a single miRNA has the potential to manifest as widespread effects on mRNA regulation and ultimately protein translation. 


\section{miRNAs and oligodendroglia}

The role of miRNAs in post-transcriptional and translational regulation has been widely described in oligodendroglia in vitro (Lau et al., 2008; Dugas et al., 2010) and in vivo (Zhao et al., 2010). Moreover, Lau et al. (2008) described that not only are there distinct cell type-specific miRNA expression profiles (oligodendroglia versus other CNS cellular lineages), there are also distinct stage-specific expression profiles within the same cellular lineage (A2B5 ${ }^{+}$ expressing versus $\mathrm{GalC}^{+}$expressing oligodendroglia isolated from the developing rat brain).

Multiple in vivo studies have identified miRNAs miR-34a, -155 and -326 as being dysregulated in active versus inactive MS lesions (Junker et al., 2009). Yet, the majority of miRNAs exhibiting the most robust changes are due to other nonoligodendroglial cell types (mainly reactive astrocytes and inflammatory cells; Junker et al., 2009). Following spinal cord injury (SCI), a disease typically associated with OL loss and limited OPC remyelination, miRNA dysregulation is observed within the epicenter of the injured rat spinal cord relative to sham controls (Liu et al., 2009). However, these studies performed the miRNA analysis on whole tissue samples and lack cell specificity. Zhao et al. (2010) demonstrated the importance of oligodendroglial-specific miRNAs during zebrafish development by dicer ablation which abolishes all miRNA processing and maturation but this does not establish which individual miRNAs are involved 
and what their expression profile is. Few studies have reconciled the need for cell specificity by assessing oligodendroglial miRNAs in monoculture. Specifically, two papers simultaneously released in 2010 each identified miR-338 and miR219 as being crucial to oligodendroglial function in several in vitro models (Dugas et al., 2010; Zhao et al., 2010). Taken together, these data suggest that although it is likely that there are miRNA expression profiles common to many if not all myelinating processes, it is reasonable to assume that there are also distinct miRNAs involved which specifically regulate various OP-behaviors, including but not limited to: proliferation, migration, fate-commitment, context specificity, differentiation and remyelination.

\section{Summary of experimental directions}

Genetic mouse models are powerful tools to address specific mechanistic questions about disease pathology and treatment when coupled with other models of demyelination as $\mathrm{SCI}, \mathrm{TBI}$ and $\mathrm{EAE}$. The rat model of ethidium bromide (EB) demyelination has been used extensively to investigate the loss of myelin and glia, the post-demyelination inflammatory response and remyelination (Blakemore and Franklin, 2008). However, rats carry little to no potential for genetic manipulation. Therefore, we attempted to develop a model of EB demyelination in the mouse. Chapter 2 addresses the similarities and differences between the rat and mouse following EB injection into the thoracic spinal cord. 
Additionally, the importance and distribution of locomotor pathways within mammalian spinal white matter is reinforced.

Past studies have demonstrated the ability to accurately assess rodent ventral locomotor pathways using in vivo electrophysiology. Targeting stimulation to subcortical areas or the brainstem, a transcranial magnetic motor-evoked potential (tcMMEP) response can be elicited and recorded from the hindlimbs. However, studies initially characterizing tcMMEP responses in mice were elicited with a custom prepared magnetic stimulator not commercially available (Hill et al., 2009). Therefore, Chapter 3 addresses the fact that unlike in the rat, mouse tcMMEP response assessment in mice is unreliable using the current commercially available technology.

Following demyelination, there is a population of mitotically competent OPs which are harbored in the CNS throughout adulthood and are well known to possess the potential to successfully remyelinate during myelin maintenance (lester et al., 1997) and in select animal models (Blakemore and Franklin, 2008). Therefore, the development of therapeutic targets within OPs which may facilitate remyelination in diseases where the process is inhibited or abolished is of high clinical priority. Chapter 4 addresses the role of microRNAs (miRNAs) in remyelinating OPs and identifies potential therapeutic miRNA targets by which remyelination can be improved. Lastly, in Chapter 5 we will address the overall 
conclusion of all data presented as well as the clinical implications and therapeutic potential moving forward. 
Figure 1. miRNA-induced translational repression of target mRNA. Schematic demonstrating that in the absence of miRNA expression, target transcripts are translated to protein $(A)$. Following induction, miRNAs assembly into the miRISC thereby directing translational repression via base pairing to complementary sequences on target mRNAs (B). 
A

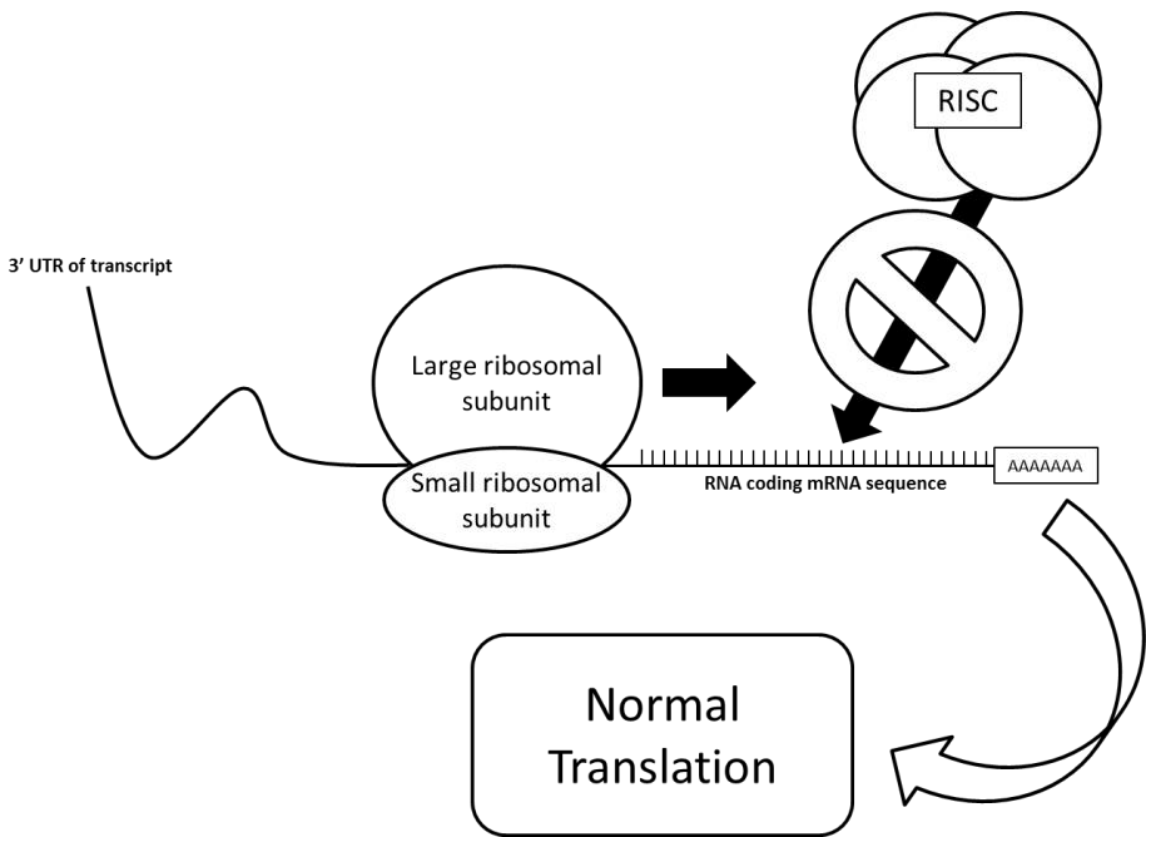

B

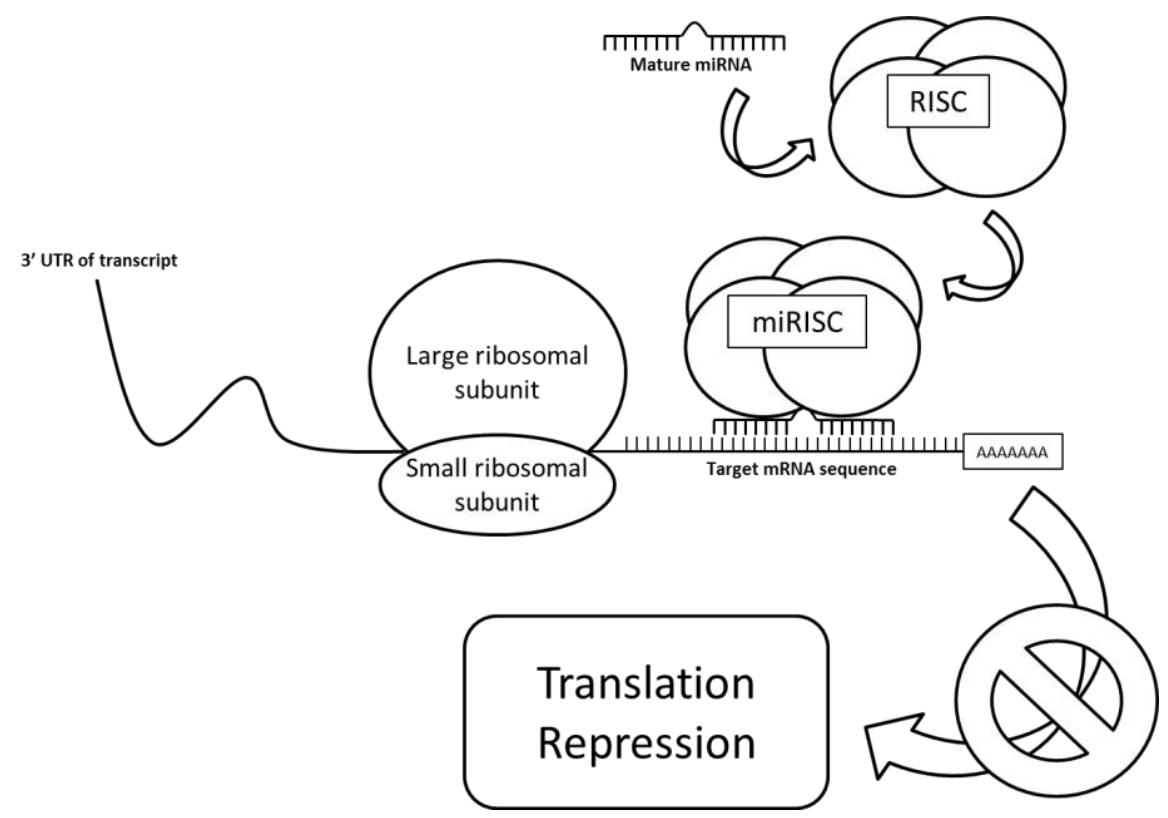




\section{CHAPTER 2}

\section{FUNCTIONAL CONSEQUENCES OF ETHIDIUM BROMIDE DEMYELINATION OF THE MOUSE VENTRAL SPINAL CORD}

\section{Introduction}

Ethidium bromide (EB) has been used extensively in the rat as a demyelinating model to assess endogenous remyelination (Blakemore \& Franklin, 2008), remyelination by engrafted cells (Tetzlaff et al., 2011; Enzmann et al., 2006) and to identify white matter tracts responsible for locomotion (Loy et al., 2002a,b). Both OL and astrocyte loss are hallmarks within the epicenter of an EB lesion while axons remain unaffected (Blakemore, 2005). The mechanism of selective glial death has been suggested to occur through EBs action as a minor-groove DNA intercalator (Blakemore, 1982; 1984). However, other evidence suggests that while EB does intercalate both chromosomal and mitochondrial DNA (mtDNA), it only affects transcription of mtDNA (Hayakawa et al., 1979;

Desjardins et al., 1985; Hayashi et al., 1990). Therefore, it is likely that EB injection into white matter compromises mtDNA transcription in all cells local to the lesion with neurons and endothelial cells being preferentially less sensitive than glia in rat models. 
Past literature has reported the acute use of EB into the dorsal columns of the adult mouse, but the EB concentrations were low and no chronic time-points were used to assess remyelination status (Fushimi \& Shirabe, 2002; 2004). Considering the potential of genetic mouse models in assessing mechanism(s) of remyelination, we set out to characterize the cellular and behavioral responses to EB injection into the adult mouse spinal cord. To understand how ventral motor pathways modulate/control hindlimb locomotion in the mouse, demyelination was targeted to the ventrolateral funiculus (VLF) and ventral column (VC), together referred to as ventral white matter (VWM). In the rat, multiple ventral and dorsal pathways contain descending information involved in locomotion and functional deficits are only observed when more than one pathway is lost (Jordan, 1986; Jordan, 1991; Loy et al., 2002a,b; Schucht et al., 2002; Noga et al., 1991; Steeves and Jordan, 1980). Here, we additionally asked whether the organization of ventral motor pathways in the mouse is similar to the rat.

\section{Materials and Methods}

Animals. All animal procedures were performed in strict accordance with the Public Health Service Policy on Humane Care and Use of Laboratory Animals, Guide for the Care and Use of Laboratory Animals (Institute of Laboratory Animal Resources, National Research Council, 1996), and with the approval of the University of Louisville Institutional Animal Care and Use Committee. A total of 116 female C57BI/6 mice 6-8 weeks of age were used for all experiments 
(Charles River; Wilmington, MA). For the initial injection parameter pilot experiment, each group contained 4 mice $(0.15 \mathrm{mg} / \mathrm{mL} E B, 0.20 \mathrm{mg} / \mathrm{mL} E B, 0.25$ $\mathrm{mg} / \mathrm{mL}$ EB and saline). To characterize the effect of $0.20 \mathrm{mg} / \mathrm{mL}$ EB or saline, a total of 48 mice were used and allowed to recover for 2 weeks ( $n=11 E B, n=9$ saline) or 8 weeks post-injection ( $n=16 \mathrm{~EB}, \mathrm{n}=12$ saline). A total of 10 mice were used for EM imaging ( $\mathrm{n}=2 /$ group; saline or $1,2,3,4$ or 6 weeks post $0.20 \mathrm{mg} / \mathrm{mL}$ $\mathrm{EB})$.

Surgery. Mice were anaesthetized with 2.0\% avertin (2,2,2-tribromoethenol in 1.25\% 2-methyl-2-butanol in saline), treated with $1 \mathrm{cc}$ sterile saline (s.c.) and lacrilube for the eyes. All surgery and post-op procedures were performed on a $37^{\circ} \mathrm{C}$ heating pad. Injections were performed using a $45^{\circ}$ beveled glass micropipette (internal diameter $\sim 25 \mu \mathrm{m}$ ) driven by a Parker II picospritzer (Pine Brook, NJ). After a laminectomy and durectomy at the T10 vertebral level, mice were placed in a stereotaxic device and two $0.25 \mu$ l boluses of either EB (at concentrations of $0.15 \mathrm{mg} / \mathrm{mL}, 0.20 \mathrm{mg} / \mathrm{mL}, 0.25 \mathrm{mg} / \mathrm{mL} E B$ in saline) or saline spaced 1 minute apart were injected into 2 locations separated by $1.5 \mathrm{~mm}$ rostrocaudally within the laminectomy. Injections were bilateral, approached at an $18^{\circ}$ angle to a depth of $1.1 \mathrm{~mm}$ and centered $0.45 \mathrm{~mm}$ lateral from midline. Following injections, the needle was left in place for 3 minutes to allow the EB or saline to diffuse. Following post-op suturing and care, mice were treated prophylactically with gentamycin and a second $1 \mathrm{cc}$ bolus of sterile saline (s.c.) and placed in heated recovery cages with alpha-dry bedding. Buprenorphine $(0.05 \mathrm{mg} / \mathrm{kg})$ was 
administered twice daily for 48 hours and bladders were expressed as needed (up to 2-3 times/day). All mice experienced identical housing and recovery conditions prior to and 1 week following EB.

Characterization of Behavioral and Cellular Responses to EB. Previous studies showed the medial VLF carries signals important for locomotion in the rat spinal cord (Loy et al., 2002a,b; Cao et al., 2009). Pilot studies revealed a steep doesresponse curve for axon sensitivity to EB in mouse VWM as $0.25 \mathrm{mg} / \mathrm{mL}$ EB completely destroy axons 2 weeks post-injection whereas spinal cords injected with $0.15 \mathrm{mg} / \mathrm{mL}$ display little to no demyelination (data not shown). $0.20 \mathrm{mg} / \mathrm{mL}$ EB consistently destroyed glia within the lesion epicenter while leaving axons apparently intact at 2 weeks (Fig. 2C). To confirm accuracy of the injections, serial cross sections throughout the lesion were stained with eriochrome cyanine (EC) to visualize myelinated and demyelinated areas (Fig. 2B).

Basso Mouse Scale (BMS). The BMS (Basso et al., 2006; Engesser-Cesar et al., 2005) was used to assess hindlimb function at baseline, and weekly for 5 weeks following EB injection. Mice were scored by two raters (trained by Dr. Basso and colleagues at the Ohio State University) blinded to the experimental condition.

$\operatorname{Treadscan}^{\circledR}$. We utilized the Treadscan ${ }^{\circledR}$ system (Clever System Inc., Reston, VA) to assess gait, coordination and paw rotation during forced locomotion (Beare et al., 2009; Saraswat Ohri et al., 2011). Treadscan ${ }^{\circledR}$ related measures 
analyzed were: run speed, hindlimb rotation, regularity index $(\mathrm{RI})$, total coupling and body rotation. Run speed was the maximum speed mice were able to maintain optimally coordinated locomotion. Hindlimb rotation and body rotation were calculated as absolute degrees from midline. Total coupling was calculated as follows: the percent deviation (absolute value) from naïve mice from homolateral, homologous and diagonal coupling measures summed together. Hindlimb rotation, body rotation and $\mathrm{RI}$ values were obtained manually. Treadscan ${ }^{\circledR}$ analysis was performed terminally, immediately prior to tissue processing.

Tissue Processing. Mice were sacrificed with an overdose of avertin (2.0\%) and transcardially perfused first with cold phosphate buffered saline (PBS) until the liver was clear and subsequently with cold 4\% paraformaldehyde (PFA) in PBS. Spinal cords were dissected and allowed to post-fix for 24 hours in 4\% PFA in PBS, then cryoprotected in $30 \%$ sucrose in PBS for 72 hours, both at $4^{\circ} \mathrm{C}$. Spinal cords were blocked in tissue freezing medium (Triangle Biomedical Sciences, Durham, NC) and frozen at $-20^{\circ} \mathrm{C}$ prior to sectioning with a Leica cryostat (Buffalo Grove, IL) at $20 \mu \mathrm{m}$. Sections were transferred to coated slides and stored at $-20^{\circ} \mathrm{C}$.

EC Staining. Tissue sections were stained with EC to distinguish myelinated from unmyelinated spinal cord axons. The percentage of spared white matter (SWM) surface area from an entire cross-section at the level of injection from EB injected 
mice relative to saline injected control mice was calculated as described previously (Magnuson et al., 2005; Cao et al., 2010; Saraswat Ohri et al., 2011). Brightfield images were captured with a Nikon Eclipse TE 300 inverted microscope and a spot CCD camera. White matter was traced with Nikon Elements software and epicenters were defined as the segment(s) with the least SWM. Percent SWM was calculated by comparing these numbers to identically processed control spinal cord at the identical spinal level. For ventral SWM analysis, regions of interest (ROIs) were defined as the ventral half of the cord with the equatorial line centered on the central canal (Fig. 3B).

Immunohistochemistry $(\mathrm{IHC})$. To characterize the cellular effect of EB or saline injections following 2 or 8 weeks of recovery, animals were sacrificed, perfused, fixed and spinal cord tissue processed as described above. To determine the extent of inflammation within the lesion epicenter, IHC against the common leukocyte antigen CD45 was used at 2 weeks ( $n=11 E B, n=9$ saline) and 8 weeks ( $n=9 E B, n=8$ saline). Hoechst ${ }^{T M}$ was used to identify nuclei. To determine the epicenter ROI on IHC sections, an adjacent EC stained section $(<100 \mu \mathrm{M}$ away) was superimposed over the $\mathrm{IHC}$ section in Photoshop ${ }^{\mathrm{TM}}$ and made partially transparent so the EC epicenter ROI could be traced over the appropriate area. To determine the effect of $0.20 \mathrm{mg} / \mathrm{mL}$ EB on axonal patency, sections were stained with primary antibodies against NF-M to assess axon number and diameter within the epicenter. Staining protocols were as follows: tissue sections were blocked with $5 \%$ bovine serum albumin (BSA), 10\% normal 
donkey serum (NDS), and $0.1 \%$ Triton-X-100 in tris-buffered saline (TBS) for $1 \mathrm{~h}$ at room temperature. Primary polyclonal antibodies neurofillament M (NF-M) (rabbit, 1:250, Millipore; Billerica, MA), glial fibrillary acidic protein (GFAP; rabbit, 1:500, Dako; Carpinteria, CA), CD45 (rat, 1:500, Millipore) and myelin basic protein (MBP; rat, 1:250, Millipore) were incubated for $24 \mathrm{hrs}$ at $4^{\circ} \mathrm{C}$ with $5 \%$ BSA, $5 \%$ NDS, and $0.1 \%$ Triton-X-100 in TBS. Sections were then washed at room temperature (x 3) with TBS and incubated with fluorescein (FITC; 1:200) and rhodamine (TRITC; 1:200)-conjugated F(ab')2 secondary antibodies (donkey, Jackson ImmunoResearch; West Grove, PA) for $1 \mathrm{~h}$ at room temperature. Species-specific IgG isotype controls were used to account for any non-specific binding or other cellular protein interactions. IHC imaging was done on a Nikon Eclipse TE 300 inverted microscope with a spot CCD camera. Exposure times remained identical across all images. To define the EB lesion epicenter ROI on IHC sections, an adjacent EC stained section ( $<100 \mu \mathrm{M}$ away) was superimposed over the IHC section in Photoshop ${ }^{\mathrm{TM}}$ and made partially transparent so that both the $\mathrm{EC}$ and $\mathrm{IHC}$ images could be visualized simultaneously to accurately line up the sections. Using the EC section as a guide, epicenter ROls were traced and ROls saved. Finally, the traced ROI file was opened along with its respective $\mathrm{IHC}$ image to allow for area and intensity analysis. A subset of mice at the 8 week time-point were sacrificed and total RNA from the epicenter collected ( $n=7 \mathrm{~EB}, \mathrm{n}=4$ saline). 
Electron Microscopy (EM). Tissue was fixed in buffered 2\% glutaraldehyde followed by $1 \%$ buffered osmium oxide, dehydrated in ethanol and embedded in LX 112 (Ladd Research Industries, Williston, VT). Appropriate areas were selected for examination and thin sectioned for EM. Thin sections were stained with uranyl acetate and lead citrate for viewing under a Philips CM-12 transmission electron.

Quantitative real time polymerase chain reaction ( $q R T-P C R)$. Total RNA was extracted from whole spinal cord epicenters of EB mice $(n=7)$ and saline mice $(n=4)$ at 8 weeks post injection with the RNeasy Mini-Kit (Qiagen; Valencia, CA) according to manufacturer's protocol. Extracted RNA was quantified with a Thermo Scientific NanoDrop 2000 (Cole-Parmer, Vernon Hills, IL) and cDNA was synthesized with $1 \mu \mathrm{g}$ of total RNA (RT2 First-Strand Kit, SA Biosciences; Valencia, CA) and used as a template on an ABI 7900HT real-time PCR instrument (Applied Biosystems; Foster City, CA) using a RT2Sybr ${ }^{\circledR}$ Green/Rox qPCR Mastermix (SA Biosciences) in technical replicates of four. Target and reference gene amplification was performed separately using RT2 qPCR Primer Assays (Qiagen) as follows: fibronectin 1 (Fn1; Mm.193099) and glyceraldehyde 3-phosphate dehydrogenase (Gapdh; Mm.343110). The RNA levels were quantified using the $\triangle \triangle C T$ method. Expression values obtained Fn1 primer assays were normalized to Gapdh expression values. Transcript levels are expressed as fold change respective to saline injected controls. 
Running Wheel (RW). Spontaneous wheel running has been demonstrated to improve outcome following contusive thoracic spinal cord injury (SCl; EngesserCesar et al., 2005; 2007). To assess potential segmental lumbar plasticity, EBinjected mice were allowed free access to a running wheel for 5 weeks post-EB. Mice were initially exposed to the wheel for 3 days and returned to non-RW cages for 1 week prior to baseline testing to allow any pre-lesion treatment effects from the RW to dissipate. All EB-injected mice received identical postoperative care and housing conditions for 1 week of recovery at which point they were randomly assigned to one of 3 groups: EB with a RW provided ad libitum ( $E B+R W ; n=12)$, EB no RW single housed (EB single; $n=9$ ) and $E B$ no RW multiple housed (EB multiple; $n=10)$. The EB single group was included to control for potential effects of housing condition on recovery as $E B+R W$ mice were individually housed. Saline treated mice were multiple housed throughout the experiment $(n=7)$. Wheel turns were magnetically monitored and a digital counter recorded data using a VitalView acquisition system and ER4000 Transponders (MiniMitter; Bend, OR) and data exported to a spreadsheet where total distance per unit time per mouse were calculated. The inside of the wheels were modified with clear vinyl (Home Depot) to provide a flat running surface and attached with two bolts. This modification is necessary to ensure that the rungs on the wheel do not exacerbate post-injury dysfunction (Engesser-Cesar et al., 2005).

Statistics. Left-right side differences were analyzed using paired t-tests where appropriate before proceeding with further statistical analyses with the right-left 
mean values. A repeated measures ANOVA with the group factor on averaged right-left values was employed for BMS and modified BMS subscore data across time. All significant group effects were followed by Tukey HSD post-hoc t-tests. For all other statistical analyses, a one-way ANOVA was used followed by Tukey HSD post-hoc t-tests where appropriate. Correlations were calculated with Pearson's coefficient.

\section{Results}

\section{EB Dose Response}

At 2 weeks post injection, EC staining of longitudinal sections revealed that 0.20 $\mathrm{mg} / \mathrm{mL}$ EB was the optimal dose for demyelination. The $0.15 \mathrm{mg} / \mathrm{mL}$ EB dose exhibited little to no demyelination and the $0.25 \mathrm{mg} / \mathrm{mL}$ dose severely damaged axons (data not shown). GFAP immunoreactivity at 2 weeks is restricted to the perimeter of the lesion (Fig. 2A-C) while EC stained cross sections confirmed the accuracy of the injections into the VWM (Fig. 2D-G). EM images reveal demyelinated axons which appear intact 2 weeks post-injection (Fig. 2H-J).

Behavioral response to $0.20 \mathrm{mg} / \mathrm{mL} E B$ injection into the adult mouse VWM

Bilateral injections of $0.20 \mathrm{mg} / \mathrm{mL}$ EB into the mouse VWM induces a chronic hindlimb deficit observed as a significant reduction in BMS scores compared to 
controls (Fig. 3A). At 1 week post-EB, scores centered around 4 to 5, indicating the ability to plantar step with little to no coordination. This deficit recovered slightly until week 3 when BMS scores plateaued at approximately 6 , indicating hindlimb coordination during overground locomotion. Subscores of all mice achieving a BMS score of 5 or above were also significantly lower in EB-injected mice as compared to controls (data not shown). EC staining revealed that at 2 weeks and 8 weeks post-EB, SWM at the lesion epicenter was $28.00 \%$ and $24.12 \%$ of saline injected mice, respectively. Terminal BMS scores plotted against SWM (\% of saline) at the epicenter for animals receiving $0.15(n=6), 0.20$ $(n=11)$ or $0.25 \mathrm{mg} / \mathrm{mL}(\mathrm{n}=5)$ EB showed a positive correlation $\left(R^{2}=0.7674 ; p<\right.$ $0.001, n=22)$. However, BMS scores and total SWM for the animals that received $0.2 \mathrm{mg} / \mathrm{ml}$ EB showed no correlation due to the narrow range of BMS scores represented. Previous work in the rat has shown that demyelination within the $\mathrm{VWM}(\mathrm{VLF}+\mathrm{VC})$ induces a chronic hindlimb impairment despite recovery of the ability to consistently weight support whereas complete loss of ventral white matter results in flaccid paralysis of the hindlimbs (Loy et al., 2002a,b). To specifically assess the role of ventral white matter in the mouse (Fig. 3B), regression analyses of all $0.20 \mathrm{mg} / \mathrm{mL} E B$ injected mice revealed a significant positive correlation between terminal BMS scores and spared VWM $\left(R^{2}=\right.$ 0.2521, $p<0.05, n=19$; Fig. 3C).

Inflammatory responses within the EB-lesioned VWM 
At 8 weeks post-injection, there was a significant increase in epicenter inflammatory cells as compared to saline, suggesting that EB induces a chronic inflammatory response that remains unresolved for at least 2 months (Fig. 4A-C). This was accompanied by a significant 4.2-fold increase in epicenter Fn1 mRNA transcript levels at 8 weeks as compared to saline injected VWM (Fig. 4D).

Chronic axonal patency after EB lesions in the VWM

NF-M immunoreactivity of cross-sections was performed to quantify axon number and diameter at the injury epicenter. At 8 weeks there was a significant reduction in the number of axons $/ \mathrm{mm}^{2}$ within the VWM to approximately $36 \%$ of saline controls (Fig. 5A-C). The mean equivalent diameters of the remaining NF-M labeled axons were significantly larger in EB-injected epicenters suggesting a preferential sparing of larger diameter axons (Fig. 5D). However, ultrastructural analysis of the lesion epicenter revealed that surviving axons were severely swollen at 6 weeks post-EB suggesting that swelling, as opposed to preferential sparing of large diameter axons, is a more likely interpretation of why the remaining axons appear larger (Fig. 5E-G). Taken together, these data suggest that in the mouse, remyelination ultimately fails due to axonal loss and dysfunction within EB-lesioned tissue as well as the presence of a chronic inflammatory response. 
Effect of spontaneous wheel running on hindlimb function post-EB

Both saline and EB-injected mice averaged approximately 2.4 miles/day on the running wheel and this was no different from the average daily distance run during the pre-injury habituation period which is similar to the 2.4-3.8 miles/day previously reported (Adlard et al., 2005). There were also no differences in weight gained/lost or SWM with running (data not shown). The BMS scores at 1 week post-injection for $\mathrm{EB}+\mathrm{RW}$ mice were higher than either the $\mathrm{EB}$ single or multiple housed mice indicating that the $\mathrm{EB}+\mathrm{RW}$ group received a less severe lesion (Fig. 6A). Therefore, we determined the change in BMS scores between week 1 and week 5 (BMS difference score), which revealed no significant difference between groups (Fig. 6B). Moreover, Treadscan ${ }^{\circledR}$ analysis revealed no significant RW-induced recovery. These data suggest that spontaneous wheel running does not improve functional outcome following chronic VWM demyelination in the mouse.

\section{Discussion}

EB-injection into the adult mouse VWM resulted in persistent demyelination and inflammation concomitant with axonal loss resulting in hindlimb dysfunction. RWinduced hindlimb recovery was not observed with the BMS and Treadscan ${ }^{\circledR}$. Although the inflammatory response and axonal loss differ in the rat and mouse, 
the mouse is similar to other mammals in the functional redundancy of descending locomotor pathways.

EB lesions in the mouse exhibit a much steeper dose-response curve as there is only a $0.1 \mathrm{mg} / \mathrm{mL}$ difference in concentration between little to no demyelination $(0.15 \mathrm{mg} / \mathrm{mL})$ and complete axon loss $(0.25 \mathrm{mg} / \mathrm{mL})$, whereas the rat exhibits a much wider range of concentrations between little demyelination and axon loss (Yajima and Suzuki, 1979; Graca and Blakemore, 1986; Crang and Blakemore, 1991; Penderis et al., 2003). In the rat, EB demyelination of the VLF induced no behavioral deficits detectable using the Basso, Beattie, and Bresnahan (BBB) Scale (Loy et al., 2002a). However, when VLF demyelination was combined with either dorsal column/corticospinal tract, ventral column or dorsolateral funiculus demyelination, mean BBB scores dropped to 15.9, 12.7 and 11.1, respectively. Consistent with those data, in the mouse, a significant reduction in BMS scores was observed 1 week post VWM EB-injection $(\mathrm{VLF}+\mathrm{VC})$ and this deficit persisted out to at least 2 months (Fig. 3A). Talbott et al. (2005) found that the epicenter of a rat VLF EB-lesion exhibited a transient inflammatory response that was completely resolved by 1 month. In contrast, the current study found increased CD45 immunofluorescence (Fig. 4A-C), increased Fn1 mRNA levels (Fig. 4D) and increased CD11b immunofluorescence (data not shown) at the EB lesion epicenter in mice out to at least 2 months suggesting a persistent inflammatory response. This is a phenotypic characteristic of fibrocytes, blood-borne immune cells which express CD45, CD11b and FN1 while lacking other lymphocyte 
specific markers (Sroga et al., 2003; Chesney and Bucala, 1997). Although differences in the inflammatory response between rats and mice have been previously suggested following spinal cord contusion (Sroga et al., 2003), this is the first evidence of this following EB-injection.

In the rat, there is no evidence of axon loss following dorsal column EB-injection at concentrations appropriate for demyelination, despite repeated episodes of focal demyelination (Franklin and Blakemore, 2008; Penderis et al., 2003). However, NF-M immunofluorescence, as well as ultrastructural analysis of epicenter cross sections confirms that EB induces axon loss in the mouse VWM (Fig. 4A) and without remaining axons, remyelination ultimately fails. Axon loss is due to one of two possibilities. The first is that EB directly affected axons resulting in axonal death and subsequent inflammation independent of EBinduced oligodendrocyte loss. The second possibility is that EB-induced glial loss resulted in inflammation which, in combination with loss of glial-derived axonal support, resulted in axonal loss. It is likely that both possibilities contribute to axonal loss in a reciprocal manner, but these data do not provide insight into which situation arises first. A better understanding of why axonal loss occurs would aid in explaining why this phenomenon is not seen in rats following VWM demyelination. Taken together, our findings of a chronic inflammatory response, axon loss and the lack of a SWM difference between 2 and 8 weeks postinjection suggests that EB lesions in the mouse VWM are non-remyelinating. It cannot be determined if remyelination would have still failed without axon loss. 
However, this scenario is unlikely using this model considering the steep doseresponse curve for EB in the mouse. Henceforth, establishing a demyelinating lesion which consistently eliminates glia without damaging axons may not be possible in the mouse using EB.

Engesser-Cesar et al. (2005) demonstrated that spontaneous wheel running resulted in significant improvements in BMS scores following a dorsal thoracic spinal contusion. Given the permanent loss of descending ventral motor pathways and its concomitant behavioral deficit in the mouse, we hypothesized that spontaneous wheel running might partially restore hindlimb function of EBinjected mice by enhancing plasticity of intrinsic lumbar circuitry independent of remyelination. This was not observed in either the BMS or Treadscan ${ }^{\circledR}$ which is more sensitive to milder injuries (Beare et al., 2009) suggesting differences in recovery between contusive SCI and VWM demyelination. The lack of additional improvement in the RW group may be due to inherent differences in the injury model (dorsal contusion vs ventral demyelination) and/or there may be a ceiling effect rendering RW training unable to provide recovery additional to the recovery provided by in-cage activity alone (Fouad et al., 2000; Kuerzi et al., 2010). If the latter explanation is correct, this suggests that the greater initial (i.e. 1 week) hindlimb function in EB lesioned mice allowed for high levels of in-cage activity regardless of RW status. Taken together, these data offer two suggestions. The first is that RW does not facilitate lumbar plasticity robust enough to induce recovery in mice following EB-induced VWM demyelination. The second is that 
the RW is unable to improve hindlimb dysfunction in mice beyond what in-cage training alone achieves following ventral lesions which do not limit the initial capacity for extensive in-cage activity. A similar ceiling for in-cage training in the rat has been suggested with multiple additional training regimens (Fouad et al., 2000; Magnuson et al., 2009; Alluin et al., 2011; Kuerzi et al., 2010).

These data suggest that mice are similar to the rat and cat in the anatomical distribution and functional redundancy of descending locomotor pathways. There are two main polysynaptic pathways regulating the initiation of locomotion. The primary locomotor pathway originates in the mesencephalic locomotor region (MLR), projects to the medullary reticular formation (MedRF) and subsequently descends through the VLF where it terminates at the spinal locomotor centers (Noga et al., 1991; Noga et al., 2003; Jordan et al., 2008). The second originates in the pontomedullary locomotor region (PLR) and is suggested to integrate sensory trigeminal information from the propriospinal system with the spinal locomotor system (Noga et al., 1991). The PLR projects to the spinal locomotor centers either by converging with the MedRF-VLF pathway or via thedorsolateral funiculus (DLF; Noga et al., 1991; Noga et al., 2003). The work by Loy et al. (2002a) also suggests a role for the VC in locomotion as combined VLF-VC demyelination induced a significant behavioral deficit in rats which was greater than that seen following VLF demyelination alone, potentially by disrupting long propriospinal interneurons. Those data are consistent with the VWM lesions described here which resulted in chronic behavioral deficits suggesting that 
multiple locomotor pathways have been lost. Moreover, once BMS scores plateaued at approximately week 3 (Fig. 3A), locomotion was mostly coordinated (as defined by BMS and Treadscan ${ }^{\circledR}$ analysis) suggesting the presence of secondary / tertiary locomotor pathways located within the dorsal and dorsolateral region of the spinal cord.

Therefore, as in the rat and cat, the mouse has functionally redundant locomotor pathways able to compensate when other locomotor pathways are lost. However, as more descending motor pathways are lost, compensation becomes limited and locomotor impairments are observed. This is not surprising considering the conservation of these pathways across mammals and in birds (Jordan et al., 2008; Noga et al., 1991; Loy et al., 2002a; Vilensky et al., 1992; Schucht et al., 2002; Sholomenko and Steeves, 1987) and supports the importance of ventral pathways to locomotion suggesting that at least some VWM must be spared for compensation to occur. To our knowledge, this is the first description of ventral locomotor pathway function in mice. Given that infarction to the ventral portion of the cord (anterior cord syndrome; ACS) is far more common than infarction to the dorsal portion of the cord (posterior cord syndrome; PCS) following human SCI (Fehlings et al., 2012; Fulk et al., 2007; Schneider et al., 2010), the functional deficits observed with this VWM lesion may better-reflect what is observed clinically. Moreover, recovery from PCS is common while ACS related deficits are chronic as patients experience little recovery (Fehlings et al., 2012; Kirshblum and O'Conner, 1998; McKinley et al., 2007). Although these data support the use 
of vental lesions in modeling human SCI, EB-induced VWM demyelination in the mouse may not be the most optimal model to achieve this.

\section{Acknowledgements}

We thank Darlene Burke for assistance with statistical analyses, Kariena Andres for animal care, Christine Yarberry for surgical support, Kim Cash for post-

operative animal care, Johnny Morehouse for assistance with Treadscan ${ }^{\circledR}$ analysis and William DeVries and Minh Tran for assistance with tissue processing, histology and image quantification. Supported by NS054708, RR15576/GM103507, Norton Healthcare, Friends for Michael, Kentucky Spinal Cord and Head Injury Research Trust, and the Commonwealth of Kentucky Challenge for Excellence.

Authors declare no conflict of interest 
Figure 2. IHC, EC staining and EM images assess astrocytic reactivity, demyelination and ultrastructural integrity, respectively. Longitudinal sections stained with Hoechst and GFAP to identify cell nuclei (A) and astrocytes (B), respectively, display GFAP immunoreactivity restricted to the perimeter of the lesion at 2 weeks post- $0.20 \mathrm{mg} / \mathrm{mL}$ EB into the mouse VWM (C). Hoechst staining reveals a large number of nuclei within the lesion epicenter $(A)$, which were later confirmed to be infiltrating inflammatory cells (Fig. 4). EC stained cross sections at the level of injection: Saline (D) and $0.20 \mathrm{mg} / \mathrm{mL} E B-1 \mathrm{~mm}(E)$, at the epicenter $(F)$ and $+1 \mathrm{~mm}(\mathrm{G})$. EM images of $(\mathrm{H})$ naïve axons which display thick compact myelin sheaths and $0.20 \mathrm{mg} / \mathrm{mL}$ EB-injected VWM which reveal spared, demyelinated axons at $1(\mathrm{I})$ and $2(\mathrm{~J})$ weeks post-injection. Scale bars in $\mathrm{C}$ and $\mathrm{J}$ $=1000 \mu \mathrm{m}$ and $10 \mu \mathrm{m}$, respectively. 

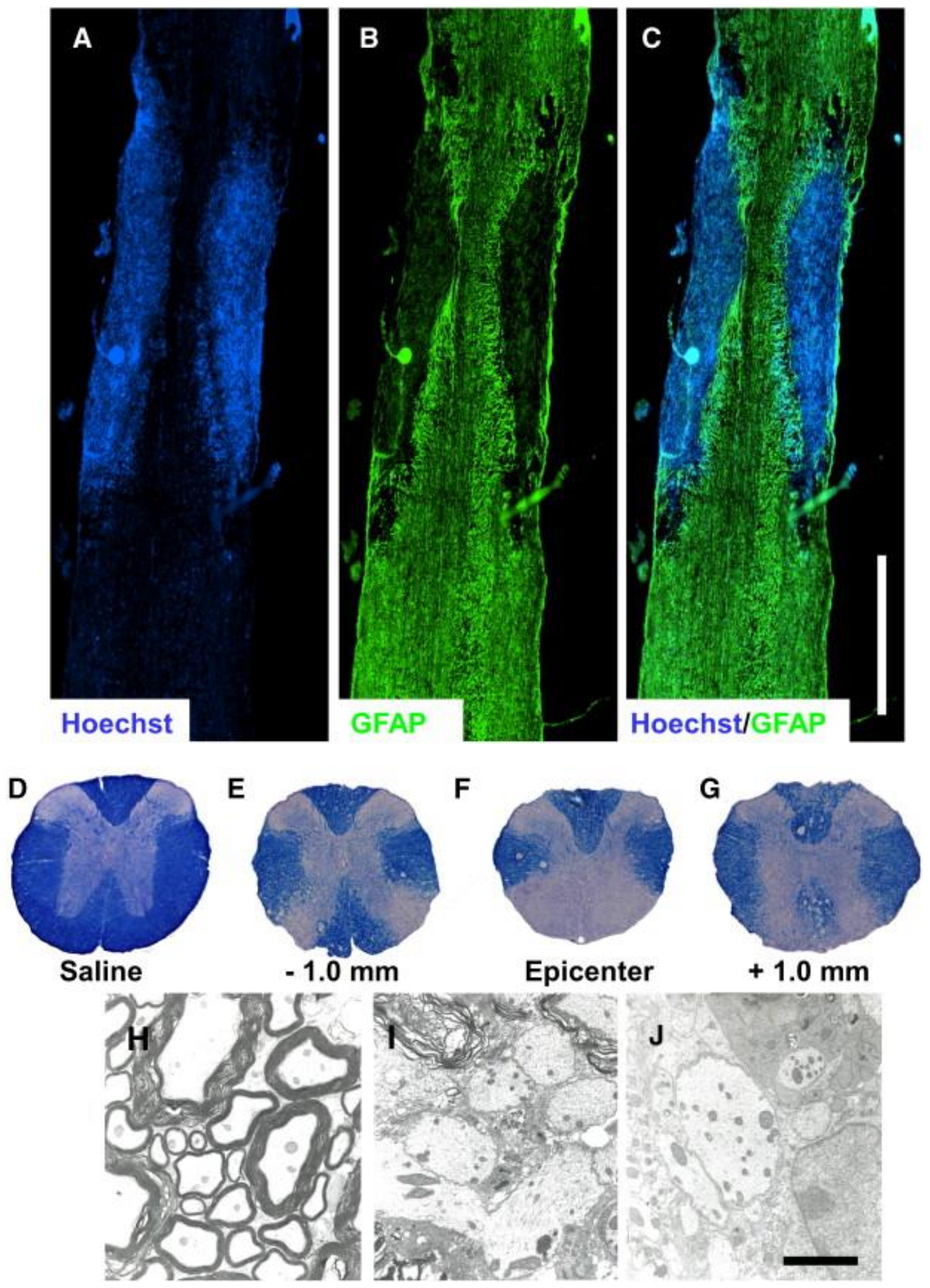
Figure 3. Functional and histological consequences of EB lesions. BMS scores reveal a significant hindlimb dysfunction which persists chronically following injection of $0.20 \mathrm{mg} / \mathrm{mL}$ EB into the mouse VWM (saline $n=9 ; 0.20 \mathrm{mg} / \mathrm{mL} E B$ $n=11 ; B)$. A positive correlation $\left(C ; R^{2}=0.2591 ; p<0.05 ; n=19\right)$ exists when terminal BMS scores were plotted against ventral spared VWM (B) highlighting the importance of ventral white matter to hindlimb function following injection of $0.20 \mathrm{mg} / \mathrm{mL}$ EB $(\mathrm{C})$. Data in A are presented as mean \pm standard deviation; ${ }^{*} \mathrm{p}<$ $0.05,{ }^{* *} \mathrm{p}<0.01$. 

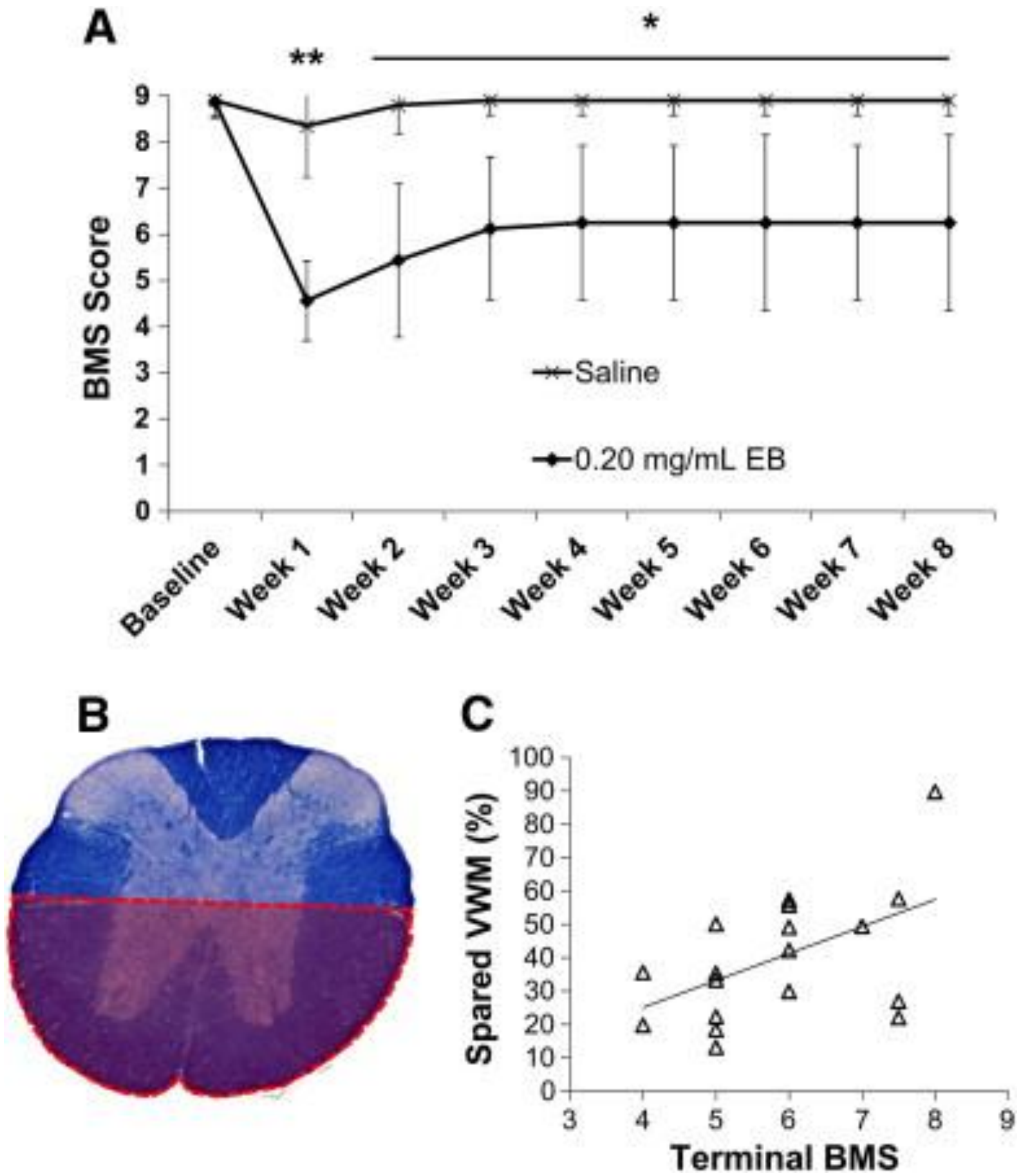
Figure 4. Chronic inflammatory response following EB lesions. There was a significant increase in $\mathrm{CD} 45^{+}$expression within the EB-injected epicenter (A) as compared to saline (B) suggesting that EB induces a chronic inflammatory response that persists for at least 2 months post-injection (saline $n=8,0.20$ $\mathrm{mg} / \mathrm{mL} E B \mathrm{n}=9 ; \mathrm{C}$ ). This was accompanied by a significant 4.29 -fold increase in epicenter Fn1 mRNA levels (normalized to Gapdh) at 8 weeks as compared to saline injected VWM (saline $n=4,0.20 \mathrm{mg} / \mathrm{mL} E B \mathrm{n}=7$; D). Adjacent $E C$ images (upper right corner of $A$ and $B$ ) were used to select ROls within the VWM for CD45 IHC (A and B). Data in C and D are mean \pm standard deviation; ${ }^{*} p<0.05$; ${ }^{* *} p<0.01$. Scale bar in $B=50 \mu \mathrm{m}$ 

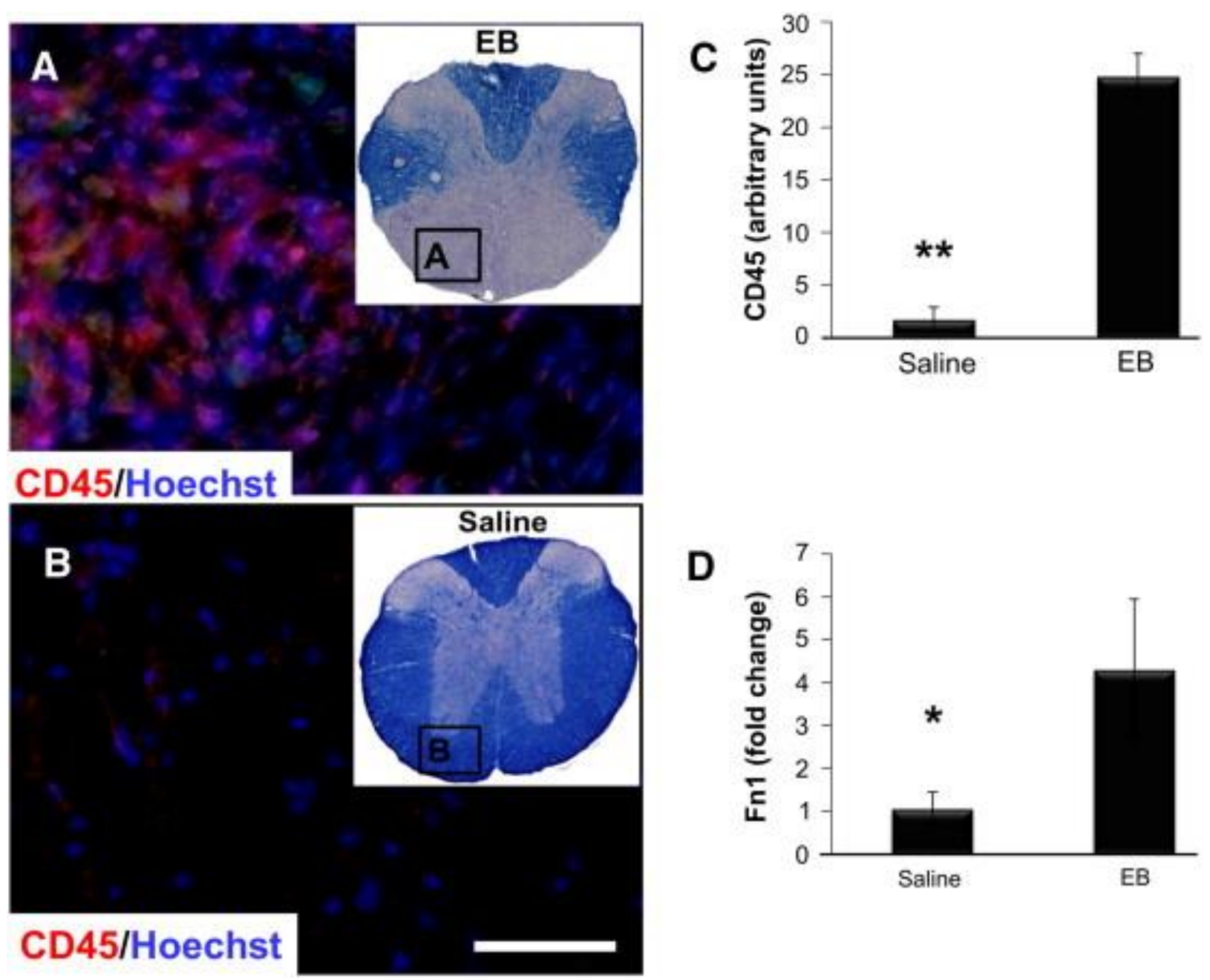
Figure 5. Chronic axonal integrity following VWM EB lesions. NF-M expression at 8 weeks (B) revealed a significant reduction in axons $/ \mathrm{mm}^{2}$ within the VWM to approximately $36 \%$ of saline controls (saline $n=8,0.20 \mathrm{mg} / \mathrm{mL} E B \mathrm{n}=9 ; \mathrm{C}$ ). Of the remaining NF-M labeled axons, mean equivalent diameters were significantly larger in EB-injected epicenters (D). Ultrastructural analysis revealed that compared to saline-injected (E) epicenters surviving axons after EB were severely swollen and much of the remaining myelin disorganized at both $3(F)$ and $6(\mathrm{G})$ weeks post-EB, accounting for the significant increase in spared axon diameter observed in $\mathrm{D}$. Data in $\mathrm{C}$ and $\mathrm{D}$ are mean \pm standard deviation; ${ }^{*} \mathrm{p}<$ 0.05 . Scale bars in $B$ and $G=50 \mu \mathrm{m}$ and $10 \mu \mathrm{m}$, respectively. 

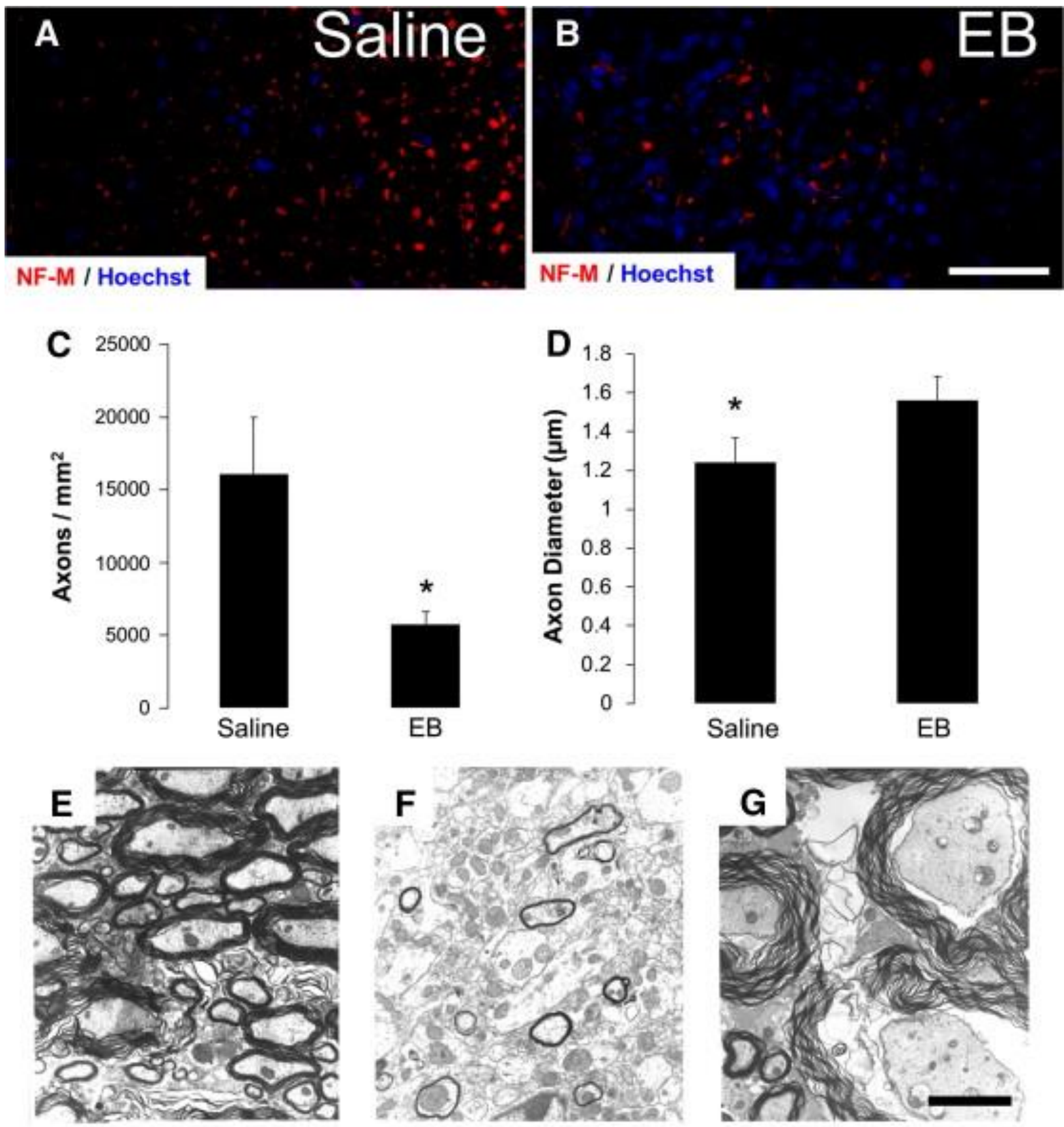

Control
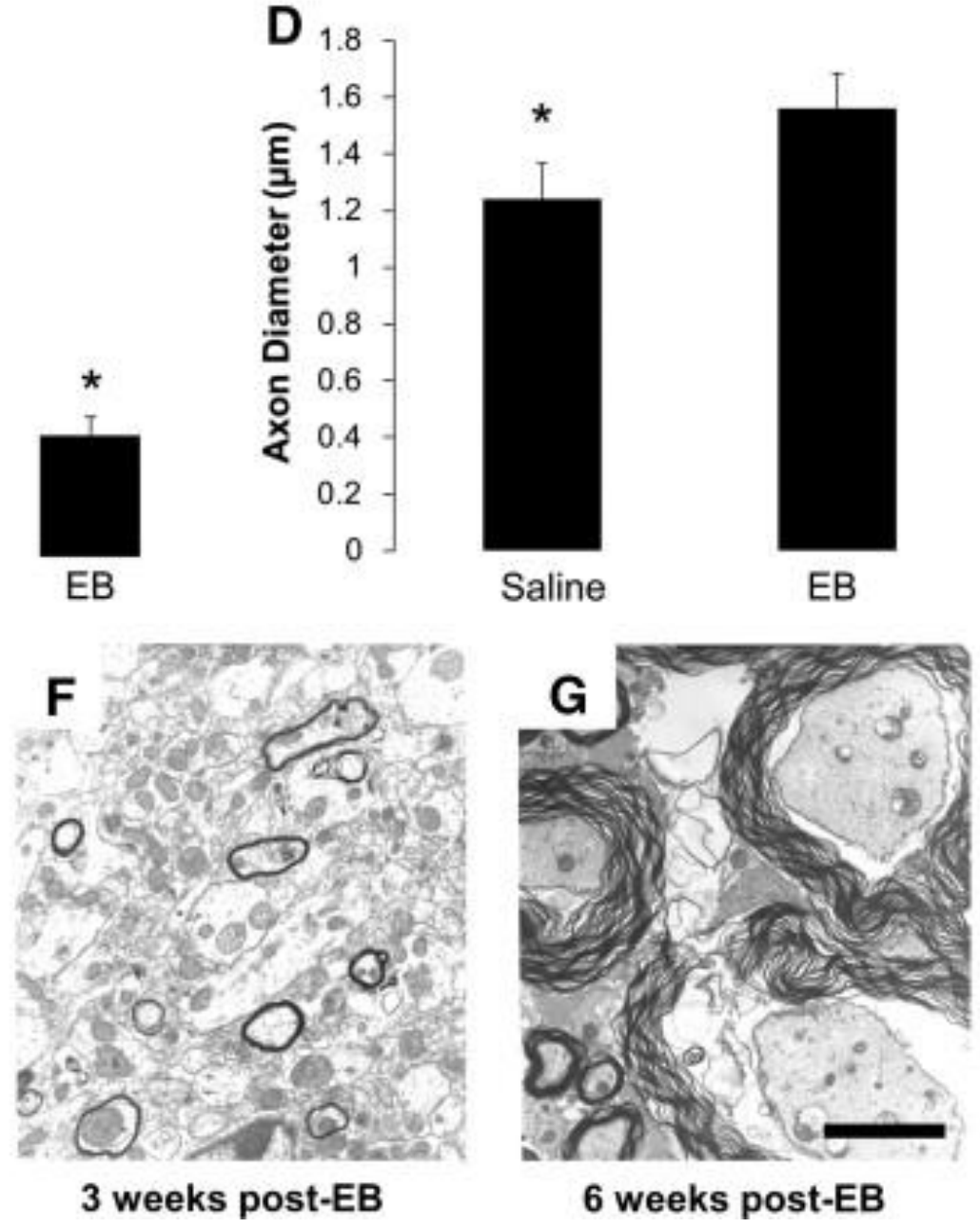
Figure 6. Neither spontaneous wheel running nor group housing improves hindlimb function following injections of $0.20 \mathrm{mg} / \mathrm{mL} E B$. EB+RW mice displayed less severe deficits prior to assignment to experimental condition (i.e. EB+RW, single or multiple housed; A). Difference scores (absolute value of week 1 BMS score - week 5 BMS score) similarly revealed no hindlimb recovery between EBinjected groups (B). Data are means \pm standard deviation, $E B+R W, n=12$; $E B$ single, $n=9$; EB multiple, $n=10$; Saline, $n=7$. 
A

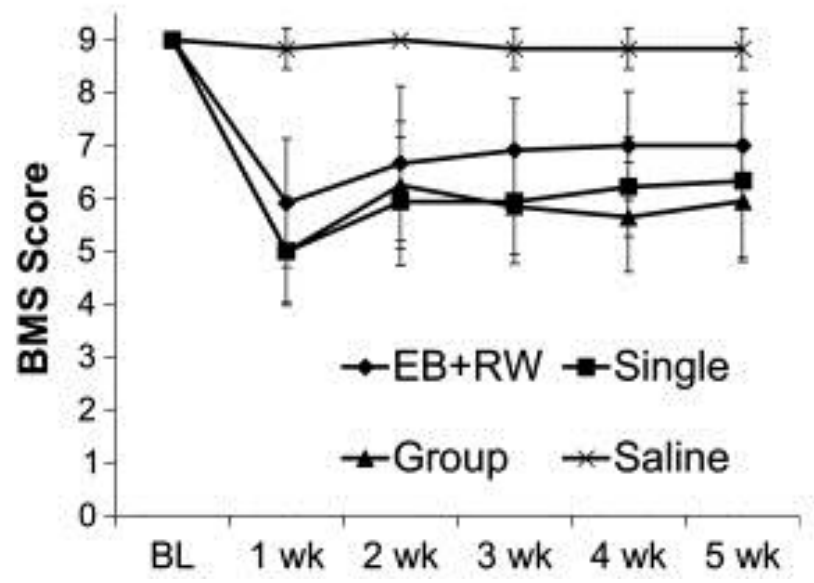

B

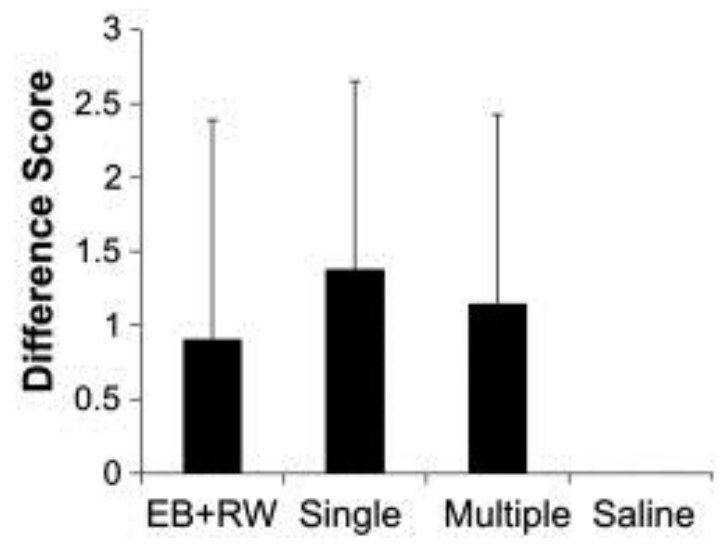




\section{CHAPTER 3}

\section{CHARACTERIZING IN VIVO ELECTROPHYSIOLOGICAL ASSESSMENT IN THE ADULT MOUSE SPINAL CORD}

\section{Introduction}

Demyelination from contusive spinal cord injury (SCI) and diseases such as multiple sclerosis (MS) results in chronic functional (Waxman, 1989; Miller et al., 2002). Injection of the gliotoxin ethidium bromide (EB) has been used extensively to model spinal cord demyelination in both rats and mice (Blakemore and Franklin, 2008; Kuypers et al., 2013; Fushimi \& Shirabe, 2002; 2004). While oligodendrocyte $(\mathrm{OL})$ loss is a hallmark characteristic observed within the epicenter of an EB lesion (Blakemore and Franklin, 2008), the absence of other glia and even some axonal loss can be observed as well (Talbott et al., 2005; Kuypers et al., 2013). This complicates data interpretation making it difficult to determine if the dysfunction observed is due to axonal or glial cell loss. Nevertheless, these studies have been instrumental in helping to identify the spinal white matter tracts responsible for particular aspects of locomotion (Loy et al., 2002a; Nichols et al., 2013; Kuypers et al., 2013) as well as assessing remyelination (Talbot et al., 2005; Talbott et al., 2007; Blakemore and Franklin, 2008). Moreover, when combined with irradiation to prevent endogenous 
remyelination, EB demyelination provides a platform to assess exogenous remyelination following engraftment of Schwann cells or pro-myelinating OLprogenitors (Franklin et al., 1996; Hinks et al., 2001).

The transcranial magnetic motor-evoked potential (tcMMEP) response (Linden et al., 1999) is a reliable functional assessment method commonly used to assess spinal cord conduction in EB-demyelinated (Loy et al., 2002a,b; Talbott et al., 2005) and spinal contused (Magnuson et al., 1999; Zhang et al., 2008) rats and spinal lacerated mice (Hill et al., 2010). The response is magnetically stimulated in the brainstem and descends through the spinal cord via the ventrolateral funiculus (VLF) where it synapses monosynaptically onto lower motor neurons controlling gastrocnemiums muscle contractions (Magnuson et al., 1999). VLF integrity is assessed by using gastrocnemius muscle contraction intensity and latency as a proxy for tcMMEP response intensity and latency. The tcMMEP response is ablated by SCI (Magnuson et al., 1999), VLF demyelination (Loy et al., 2002a,b) and thoracic transection (Hill et al., 2010). However, following SCI tcMMEP responses have been shown to return in rats engrafted with oligodendrocyte precursor cells (Cao et al., 2010).

All studies mentioned above involving tcMMEP assessment in rats have been performed with the commercially available Cadwell stimulator (Cadwell Laboratories, Kennewick, WA). There is only one published report of mouse tcMMEP response assessment (Hill et al., 2010). This study utilized descending 
thoracic lacerations which resulted in reduced tcMMEP amplitude that decreased incrementally with lesion depth but this study used a customized Magstim stimulator (The Magstim Company, UK). Considering the unlimited genetic potential of mouse models, characterizing the use of a commercially available stimulator would facilitate standardization of tcMMEP assessment in mice across laboratories would provide a valuable tool to assess mouse remyelination in vivo. Therefore, we aimed to characterize both tcMMEP response assessment and the effect of EB-demyelination on tcMMEP responses in the adult mouse using the Cadwell stimulator. Unfortunately, following EB-demyelination, axon loss was more robust in mice than it is in rats making it impossible to discern whether loss of tcMMEP responses were due to demyelination or complete axonal severance (Kuypers et al., 2013). Moreover, the presence of the tcMMEP responses in EBdemyelinated mice despite axonal loss suggested that the Cadwell stimulator might stimulate an alternate pathway in mice than it does in rats. To that end, we finally investigated the effect of descending thoracic transections and cervical lateral hemisections on tcMMEP responses recorded using a Cadwell stimulator determining that tcMMEP assessment in mice with the currently available commercial technology is unreliable.

\section{Materials and Methods}

Animal Care and General Surgical Procedures: All animal procedures were performed in strict accordance with the Public Health Service Policy on Humane 
Care and Use of Laboratory Animals, Guide for the Care and Use of Laboratory Animals (Institute of Laboratory Animal Resources, National Research Council, 1996), and with the approval of the University of Louisville Institutional Animal Care and Use Committee. Adult female C57BI/6 mice were anaesthetized with 2.0\% avertin (2,2,2-tribromoethenol in 1.25\% 2-methyl-2-butanol in saline), treated with 1cc sterile saline (s.c.) and lacrilube for the eyes. Body temperatures were maintained during all surgical and post-op procedures at $37-38^{\circ} \mathrm{C}$. Following post-op suturing and care, mice were treated prophylactically with gentamycin and a second $1 \mathrm{cc}$ bolus of sterile saline (s.c.) and placed in heated recovery cages with alpha-dry bedding. Buprenorphine $(0.05 \mathrm{mg} / \mathrm{kg})$ was administered twice daily for 48 hours. Soft food, additional subcutaneous saline injections and bladder expression was performed as needed (up to 2-3 times/day). The animals were housed in ventilated cages ( $\leq 5$ mice / cage) and provided ad libitum food and water.

Ethidium Bromide (EB) Injections: Ethidium bromide injections were performed using a beveled glass micropipette ( 25 um) driven by a Parker II picospritzer. After a laminectomy and durectomy at the T10 vertebral level, mice were placed in a stereotaxic device and two $0.25 \mu$ l boluses of either $\mathrm{EB}(0.20 \mathrm{mg} / \mathrm{mL} ; \mathrm{N}=13)$ or saline $(\mathrm{N}=9)$ spaced 1 minute apart were injected into 2 locations separated by $1.5 \mathrm{~mm}$ rostro-caudally within the laminectomy. Injections were bilateral, approached at an $18^{\circ}$ angle to a depth of $1.1 \mathrm{~mm}$ and centered $0.45 \mathrm{~mm}$ lateral from midline. This lesion produces a ventral column (VC) lesion encompassing 
both the VLF and ventral funiculus (VF; Kuypers et al., 2013). Following injections, the needle was left in place for 3 minutes to allow the EB or saline to diffuse.

Lacerations: Cervical lateral hemisections $\mathrm{C} 5 / \mathrm{C} 6(\mathrm{~N}=4)$ and complete thoracic transections at $\mathrm{T} 9 / \mathrm{T} 10(\mathrm{~N}=2)$ were performed as previously described (Hill et al., 2010). Briefly, anesthetized mice were placed into a spinal column stabilizer and lacerations were made using the Louisville Injury System Apparatus with the Vibraknife attachment (LISA-Vibraknife; Louisville, KY). Only complete transection mice received a laminectomy. Both lacerations were produced at a depth of $1.6 \mathrm{~mm}$ with the lateral hemisection only extending to midline. Following each lesion, the spinal cord was assessed for additional damage to rule out the possibility of contusion. Musculature suturing was done with a 10-0 suture and the skin stapled with 9-mm metal suture clips (Becton Dickinson, Sparks, MD).

Basso Mouse Scale (BMS) Assessment: The BMS assessed hindlimb function during open field locomotion in all lacerated mice. This scale is optimized to reliably assess mouse hindlimb function following $\mathrm{SCl}$ and other insults which compromise hindlimb function (Basso et al., 2006; Engesser-Cesar et al., 2005). BMS assessment was performed at baseline and at day 7 post-laceration. Mice were scored by two raters (trained at Ohio State University) blinded to the experimental condition. BMS scores range from 0-9 and subscores were not 
calculated as none of the lesioned animals displayed scores above 5, which functionally corresponds to coordination.

Electrophysiology: Transcranial magnetic motor evoked potentials (tcMMEPs) was used to examine conduction within the VLF bilaterally. Baseline conduction was assessed once preoperatively and weekly for 8 weeks following EB injection and at days 3, 7 and 10 post-laceration. Assessments were done in restrained, unanesthetized mice. The mice were restrained using a custom mouse restraint jacket designed by Lomir Biomedical Inc. Animals were lightly anesthetized with isoflurane to eliminate stress and facilitate fastening the animals into the restraint jacket. Animals were then placed back into their cages for 15 minutes and allowed to recover from anesthesia. A commercially available Cadwell Stimulator/recorder (MES-10, 2 tesla maximum output) attached to a $25 \mathrm{~mm}$ dual-coil hand held magnetic transducer was used for assessment of VLF conduction. The magnet was placed over the scalp and a $70 \mu$ s magnetic pulse was delivered while EMG responses were recorded from needle electrodes inserted bilaterally into the gastrocnemius muscle. Reference electrodes were placed into the distal tendon of the gastrocnemius muscle and a ground electrode was place sub-cutaneously above the base of the tail. Magnet intensity was set at $100 \%$ across all mice as intensities of $80 \%$ and $90 \%$ yielded lower amplitude responses suggesting that the ceiling of axonal recruitment has not been maximally reached. The onset latency was measured in milliseconds (msec) and the peak-trough amplitude was measured in millivolts $(\mathrm{mV})$. The 
signal-to-noise ratio was set at 5 times the level of background noise and recordings with amplitudes less than this were considered noise. The procedure takes only a few minutes and does not appear to be painful to the animals, as they tolerate the restraint well and do not show signs of discomfort during the stimulation and recording procedures. Following testing and in between animals, mice were placed into a new clean cage with topical administration of bacitracin to the puncture wounds while electrodes were cleaned and sharpened. Restraint jackets were washed between testing sessions.

Tissue Processing: Mice were sacrificed with an overdose of avertin (2.0\%) and transcardially perfused with cold phosphate buffered saline (PBS) until the liver was clear and fixed with cold $4 \%$ paraformaldehyde (PFA). Spinal cords were dissected and allowed to post-fix for 24 hours in 4\% PFA cryoprotected in 30\% sucrose in PBS for 72 hours. Spinal cords were blocked in tissue freezing medium (Triangle Biomedical Sciences, Durham, NC) and frozen at $-20^{\circ} \mathrm{C}$ prior to sectioning at cryostat (20um). Sections were transferred to coated slides and stored at $-20^{\circ} \mathrm{C}$.

Eriochrome Cyanine (EC) Staining: Tissue sections were stained EC to distinguish myelinated from unmyelinated spinal cord throughout the lesion. The percentage of total white matter in EB injected mice relative to saline injected control mice was adapted from a protocol formerly described in rats (Magnuson et al., 2005). Briefly, slides were allowed to thaw at room temperature for $20 \mathrm{~min}$. 
Then, sections were hydrated by submersion in xylene ( $60 \mathrm{~min}$ ) and hydrated by submersion in 100\% ethanol ( $3 \mathrm{~min}$ ), $95 \%$ ethanol ( $3 \mathrm{~min}$ ), $70 \%$ ethanol (3 $\mathrm{min}$ ), $50 \%$ ethanol ( $3 \mathrm{~min}$ ), and distilled water ( $2 \mathrm{~min})$. Slides were then stained with EC for 10 min. After rinsing in distilled water $(x 2)$ to wash excess EC, sections were differentiated in $0.5 \%$ ammonium hydroxide solution for $30 \mathrm{sec}$, followed by 2 more distilled water washes. Sections were air dried overnight at room temperature, cover-slipped the next day and again dried overnight at which point the tissue was ready for imaging. Brightfield images were captured with a Nikon Eclipse TE 300 inverted microscope and a spot CCD camera. White matter was traced with Nikon Elements software and epicenters were defined as the segment(s) with the least spared white matter.

Statistics: We checked for left-right side differences using paired t-tests where appropriate before proceeding with further statistical analyses with the right-left mean values. A repeated measures analysis of variance (ANOVA) with the group factor on averaged right-left values was employed for BMS, modified BMS subscore, tcMMEP amplitude and latency data across time. All significant group effects were followed by Tukey HSD post-hoc t-tests. At week one, EB+RW displayed a higher BMS score as compared to other EB injected groups suggesting that group received less severe injuries as RW treatment did not begin until 1 week post-EB. Therefore, absolute values of the difference score between weeks 1 and 5 to assess recovery following induction of wheel running. 
For all other statistical analyses, a one-way ANOVA was used followed by Tukey HSD post-hoc t-tests where appropriate.

\section{Results}

\section{$B M S$}

Open field locomotor assessment was performed at baseline and at days 2 and 7 post-laceration. Complete transection mice all displayed BMS scores of 0-1 at 2 and 7 days post injury which functionally corresponds to flaccid hindlimb paralysis with an occasional spasm and absolutely no weight barring (data not shown). Lateral hemisection mice displayed unilateral paralysis in the limb ipsilateral to the lesion. In unilaterally injured mice with the ability to achieve coordinated open field locomotion (scores above 5), the BMS is not an appropriate measure of hindlimb dysfunction as assessment requires simultaneous assessment of all 4 limbs making it more suited for bilateral injuries. However, in severely injured mice lacking the ability to coordinate (BMS scores 5), the BMS can be used to assess hindlimb dysfunction unilaterally without accounting for the unaffected limb. Therefore, in the unilateral injured lateral hemisection group, the BMS was used to assess dysfunction in the limb ipsilateral to the lesion. These mice displayed complete flaccid paralysis (BMS scores of $0-1$ ) in the affected limb and BMS scores of 2-4 for the unaffected limb. 
By day 7 , no noticeable dysfunction was present in the unaffected limb while BMS scores for the affected limb remained at 0-1 (data not shown).

tcMMEP responses

Trans cranial magnetic motor evoked potential (tcMMEP) responses were assessed as previously described in rats (Loy et al., 2002; Talbott et al., 2005). Following bilateral EB-injection into the VC, no differences were found when comparing baseline tcMMEP amplitude and latency to responses attained following EB-injection. No changes were present within group across time for either amplitude or latency (Fig. 7A,B). There was no significant correlation for either amplitude or latency verse terminal BMS score suggesting that functional outcome following EB-injection had no relationship with tcMMEP amplitudes or latencies.

\section{Lateral hemisections and complete transections}

Considering the lack of a clear difference for either amplitude or latency in the saline group as compared to any EB-injected group raised concern as to the interpretability of the tcMMEP data. Therefore, we performed laceration experiments to confirm that the observed responses in EB-injected mice were in fact tcMMEP responses. Complete transections displayed no tcMMEP responses at 3 days posy injury (dpi), but responses began to return by $7 \mathrm{dpi}$ and were no 
different than baseline responses at $10 \mathrm{dpi}$ (Fig. 8A). Similarly, ipsilateral tcMMEP responses in the lateral transected mice were abolished by 3dpi but were indistinguishable from contralateral responses at $10 \mathrm{dpi}$ while contralateral responses remained at all time-points following lateral transection (Fig. 8B). We interpret these data as artifact and not evidence of restored spinal cord conduction but rather evidence of a potential extraspinal pathway which may or may not initiate in the spinal cord, can circumnavigate the lesion site and re-enter the cord below the level of the lesion yielding motor evoked potentials in the musculature. Spinal shock post-transection or lateral transection would also explain the temporary abolishment of the tcMMEP responses at the 3 dpi timepoints and as spinal shock resolves between 5 and $10 \mathrm{dpi}$, the responses return. Moreover, considering that conduction latencies were not statistically different at baseline testing as compared to any post-transection time-point, it is likely that we are observing tcMMEP responses being conducted outside of the spinal cord in uninjured mice. Therefore, we have determined tcMMEP response assessment in awake, adult mice using a commercially available magnetic stimulator as unreliable.

White matter sparing (WMS) and gross spinal cord anatomy

EC staining of spinal cord cross sections throughout the lesion confirmed that the EB injections were accurate and demyelination was targeted to the VC (Fig. 7C). No residual white matter remained in completely transected mice or within the 
lesioned hemisphere of lateral hemisection mice (data not shown). Moreover, gross anatomy of dissected spinal cords revealed that laceration lesions did not regenerate and that lesioned pathways were still completely severed by $10 \mathrm{dpi}$.

\section{Discussion}

Assessing tcMMEP responses in mice with the currently available technology is unreliable. Although reference electrodes demonstrated gastrocnemius muscle contractility with response latencies and amplitudes that resemble tcMMEP responses (Fig. 7), they were maintained in both lateral hemisected (Fig. 8A) and complete transected mice (Fig. 8B). One possible explanation could be that unlike the rat, the small size of the mouse makes it difficult to target the magnetic stimulation to only subcortical areas. This could manifest in one of three ways: 1 . Collateral stimulation of the lower motor neurons directly; 2. Collateral stimulation of the muscle directly; 3 . Collateral stimulation of the recording electrode directly. As a positive control to recording electrode placement, the magnetic stimulator was placed below the level of EB or saline injection eliciting a high amplitude response approximately $2.0 \mathrm{~ms}$ in latency (data not shown). This confirmed that electrode placement was in fact in the gastrocnemius muscle for animals lacking a response. However, this also confirmed that responses obtained by stimulating subcortically could not be explained by any of the possibilities mentioned above. A latency of $2.0 \mathrm{~ms}$ would be appropriate for the short, monosynaptic lower motor neuron - gastrocnemius pathway. Moreover, a latency acquired by directly 
stimulating the muscle or recording electrode would be shorter than a latency acquired by directly stimulating the lower motor neuron directly. Thus, considering that responses acquired when stimulating subcortically displayed latencies of at least $5.0 \mathrm{~ms}$ (Fig. 7B), this eliminates the possibility that subcortically elicited responses were due to unintentional stimulation of other anatomical structures or recording electrodes directly.

Another possible explanation is that magnetic stimulation does elicit a response which originates subcortically, but as the response descends down the spinal cord, it exits the spinal cord to traverse the site of the lesion before entering back into the spinal cord below the lesion. One possible extraspinal bridge could be the sympathetic chain ganglia which reside in close proximity to the ventral spinal cord. However, these pathways would be multisynaptic, potentially following a complex and fortuitous route extraspinally before entering back into the cord below the lesion. Both the number of synapses and increased distance travelled of extraspinal responses suggest that response latencies would be greater than what we report here eliminating extraspinal conduction pathways as an explanation.

A second possibility is that the magnetic stimulation is stimulating axial musculature rather than particular anatomical loci within subcortical white matter. Axial musculature in the mouse is anterolaterally contiguous along the dorsal aspect of the torso and hindlimbs. Therefore, magnetic stimulation of axial 
musculature could elicit a response which traverses down to the gastrocnemius muscle thus initiating a contraction. In both cases (chain ganglia and axial musculature), the methods required to assess this would are invasive when considering that responses would need to be assessed in fully awake animals as anesthesia can interfere with conduction. Electrophysiological assessment would need to be performed prior to, immediately after surgical dissection of the extraspinal pathways and then assessed over time to truly determine if responses are abolished. Therefore, until more mouse specific tcMMEP response assessment technology is commercially available, it will be challenging to interpret results across laboratories suggesting the presence of a tcMMEP response in mice.

\section{Acknowledgements}

We thank Darlene Burke for assistance with statistical analyses, Kariena Andres for animal care, Ping Zhang and Christine Yarberry for surgical support, Kim Cash for post-operative animal care, Johnny Morehouse for assistance with BMS assessment and William DeVries, Monica Vetter, Kurtis James and Minh Tran for assistance with tissue processing, histology and image quantification. Supported by NS054708, RR15576/GM103507, Norton Healthcare, Friends for Michael, Kentucky Spinal Cord and Head Injury Research Trust, and the Commonwealth of Kentucky Challenge for Excellence. 
Figure 7. Mouse tcMMEP response assessment. No changes were present within group across time for either amplitude (A) or latency (B) suggesting that functional outcome following EB-injection had no relationship with tcMMEP amplitudes or latencies. 
A Amplitude

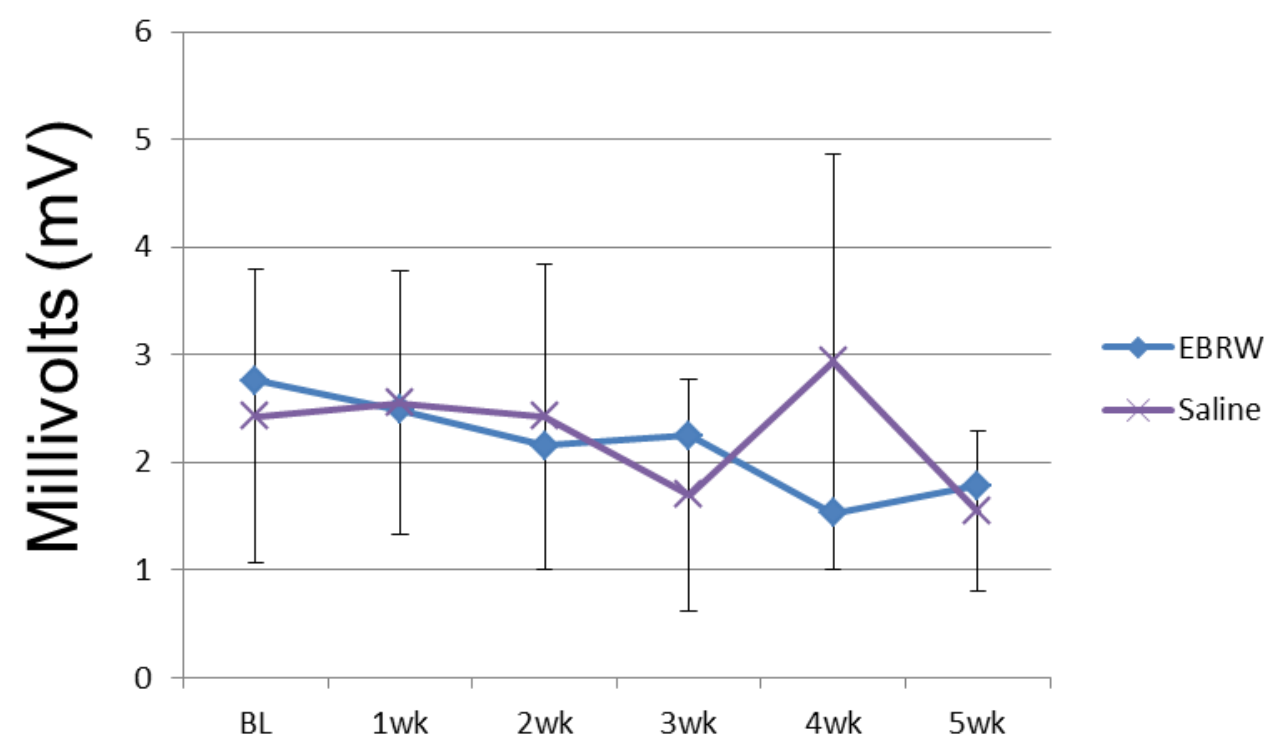

B Latency

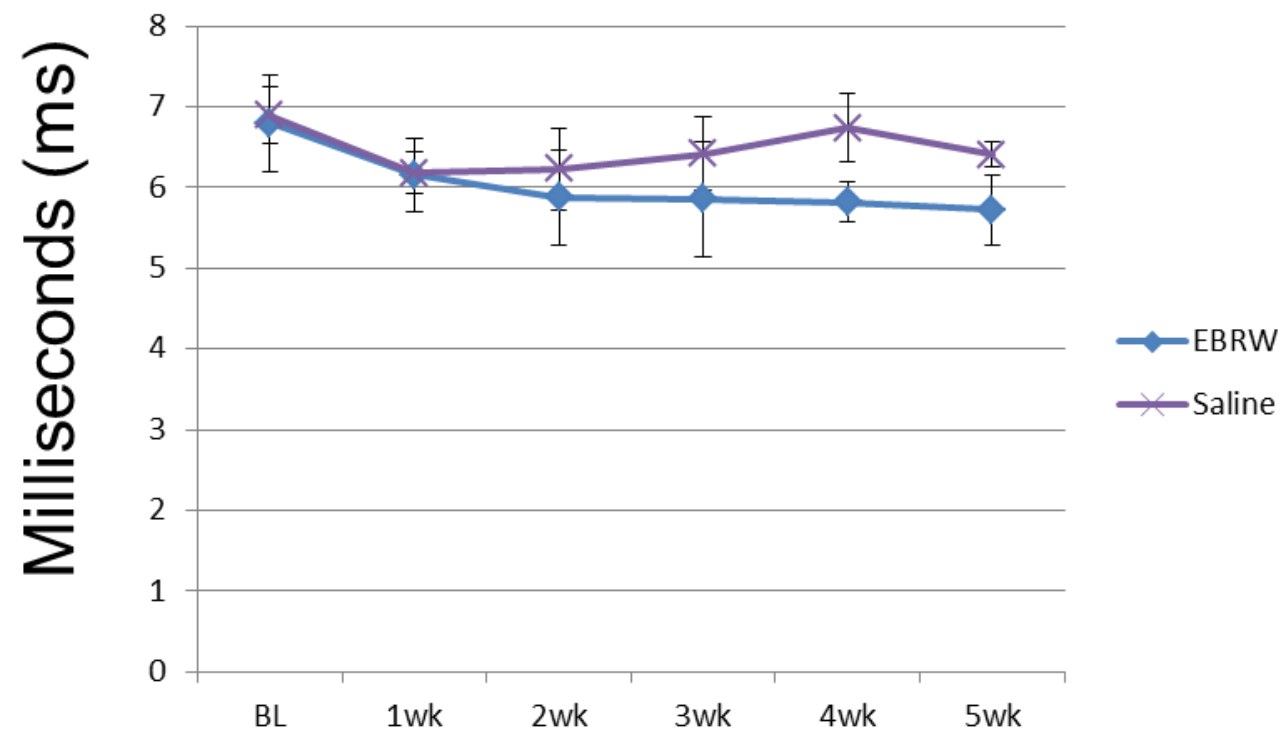


Figure 8. Spinal cord laceration does not abolish subcortically-elicited MMEP responses. Ipsilateral subcortically-elicited MMEP responses in laterally hemisected mice were abolished by 3 days post injury (dpi) but were indistinguishable from contralateral responses at $10 \mathrm{dpi}$ while contralateral responses remained at all time-points following lateral hemisection (A). Gross anatomical images in left column are of 2 laterally hemisected mice (A). Similarly, complete transections displayed no subcortically-elicited MMEP responses at 3 days posy injury (dpi), but responses began to return by $7 \mathrm{dpi}$ and were no different than baseline responses at $10 \mathrm{dpi}(\mathrm{B})$. Gross anatomical images in right column are of 2 complete transected mice $(B)$. 

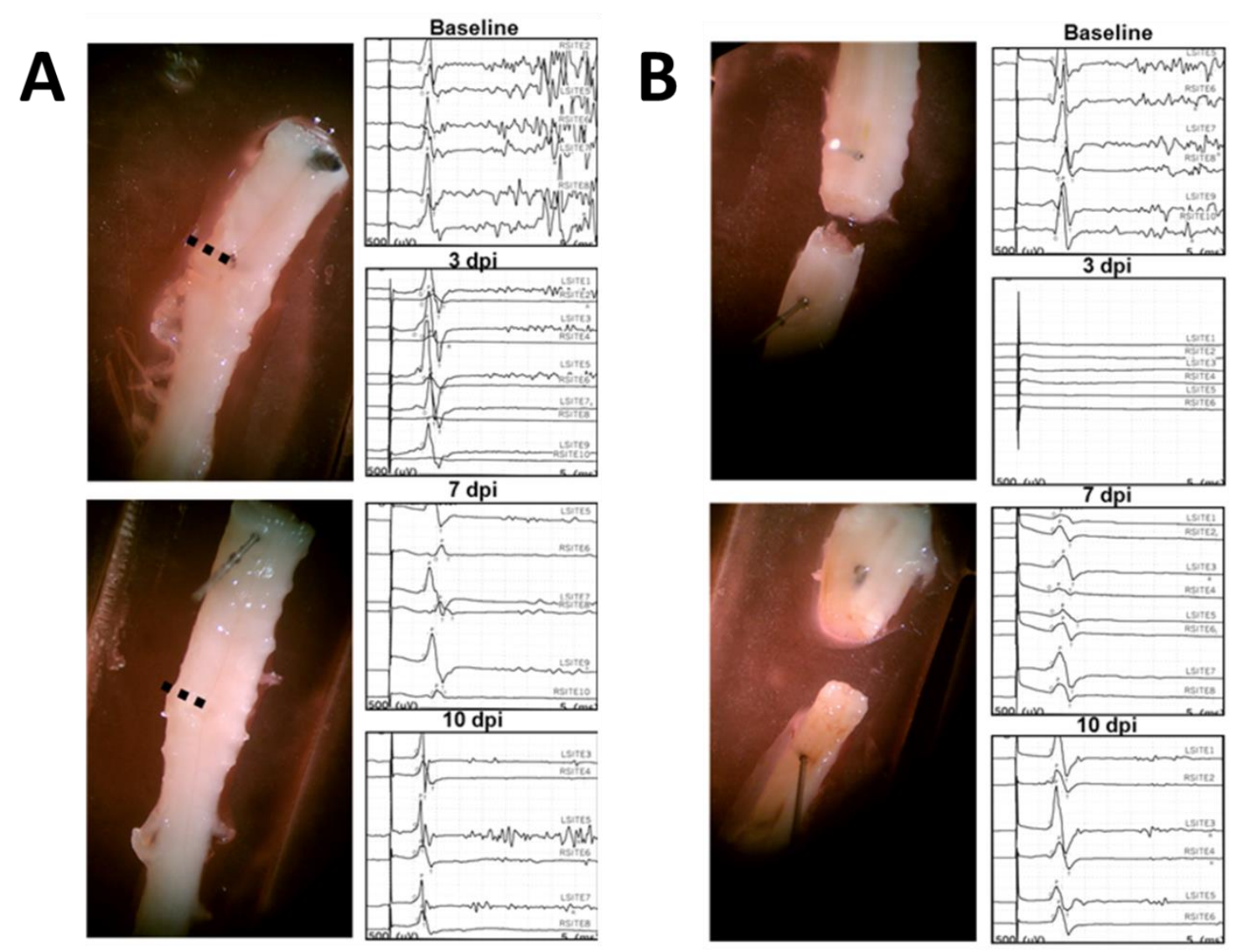


\section{CHAPTER 4}

\section{CHARACTERIZING SFMBT2 CLUSTER MICRORNA (MIRNA) MIR-297C EXPRESSION INCREASES DURING MOUSE OLIGODENDROCYTE PROGENITOR (OP) DIFFERENTIATION AND REGULATES CELL CYCLE IN VITRO}

\section{Introduction}

Oligodendrocytes (OLs) are the only source of myelin in the central nervous system (CNS) and their loss is partially responsible for the dysfunctional outcomes observed in multiple sclerosis (MS; Miller et al., 2000), the leukodystrophies (Perlman and Mar, 2012) and spinal cord injury (SCl; Waxman, 1989). Although remyelination has been documented in human MS patients (Patrikios et al., 2006), it rarely is complete (Bramow et al., 2010). Central remyelination is driven by $\mathrm{OL}$ progenitors (OPs) which remain mitotically competent within the mammalian CNS throughout adulthood with the ability to respond to normal OL loss thus maintaining myelin levels (Franklin and ffrenchConstant, 2008). However, following extensive trauma- or disease-induced demyelination, the number of OPs required to maintain functional myelin levels outweighs the number of OPs present and therefore many strategies have been attempted to increase OP numbers and enhance remyelination (Goldman and Windrem, 2006). The transcriptional events that regulate oligodendroglia during 
remyelination are well understood as key regulatory elements such as Olig, Nkx, and Sox transcription factors have been identified (Wegner, 2008). However, since the recent discovery of miRNAs, it has become more clear that posttranscriptional events are equally important to oligodendroglial translational regulation (Emery, 2010; Barca-Mayo and Lu, 2012; He et al., 2012). MicroRNAs (miRNAs) are class of $\sim 22-33$ nucleotide non-coding RNAs which by their short nature, target tens to thousands of mRNAs for degradation or translational repression thus preventing protein production (Chen and Rajewsky, 2007; Bartel, 2009). Since the development of technologies to assay miRNA expression levels, multiple laboratories have identified miRNAs involved in remyelination in vitro (Lau et al., 2008; Dugas et al., 2010; Zhao et al., 2010). However, no data to date has identified oligodendroglial-specific miRNAs in vivo.

Here, we describe a method which allows for the direct isolation of miRNAs from callosal OPs isolated in vivo and assessment of their expression levels during de- and re-myelination. Oxalic bis-cyclohexylidenehydrazide (cuprizone) is a copper chelator which can be administered to mice in chow to induce CNS demyelination, most notably in the corpus callosum (Steelman et al., 2008). The cuprizone model has been well described and is commonly employed as a 6 week on cuprizone ingestion period (demyelination) followed by a 6 week recovery period (remyelination) where mice are returned to normal chow (Torkildsen et al., 2008). This remyelination response is driven by OPs and is observed during both the later part of the ingestion phase and throughout the 
recovery (Mason et al., 2001; Matsushima and Morell, 2001). We hypothesized that miRNA changes within OPs would accompany the remyelination response both during and following cuprizone ingestion. Using transgenic mice with an enhanced green fluorescent protein (EGFP)-gene inserted downstream of both 2',3'-cyclic-nucleotide 3'-phosphodiesterase (CNPase) promoters, CNPaseEGFP expressing OPs were isolated from the white matter of mice during cuprizone-induced de- and re-myelination and corresponding miRNA expression levels were analyzed via microarray. Compared to callosal miRNA expression levels from normal mice, hundreds of miRNAs followed dynamic expression profiles which changed significantly across cuprizone treatment conditions. We identified novel miRNAs from the Sfmbt2 cluster which were demonstrated to regulate cell cycle entry.

\section{Material and Methods}

Animals. All animal procedures were performed in strict accordance with the Public Health Service Policy on Humane Care and Use of Laboratory Animals, Guide for the Care and Use of Laboratory Animals (Institute of Laboratory Animal Re-sources, National Research Council, 1996), and with the approval of the University of Louisville Institutional Animal Care and Use Committee and Institutional Biosafety Committee. CNPase-EGFP ${ }^{ \pm}$mice were received from the laboratory of Dr. Vittorio Gallo (George Washington University, DC). These mice have the EGFP gene inserted downstream of both CNPase promoters, C1 and 
C2 (Yuan et al., 2002), which has been well described as being enriched within oligodendroglia (Vogel et al., 1988). For all experiments, both male and female mice ages 8 to 10 weeks were used.

Husbandry and Genotyping. CNPase-EGFP colonies were maintained on a C57BI/6 background while experimental mice were acquired by interbreeding a homozygous sire with a heterozygous dam whose progeny are referred to as $\mathrm{CNPase}^{+}$. For genotyping, a tail sample from each pup was digested with proteinase $\mathrm{K}$ in buffer (Sigma, St. Louis, MO) at $55^{\circ} \mathrm{C}$ overnight. The following morning, DNA was extracted using the Phire Animal Tissue Direct PCR Kit (Thermo Scientific, Waltham, MA) and DNA yields were estimated with a NanoDrop 5000 machine (NanoDrop, Wilmington, DE) with DNA elution buffer used as a blank. A polymerase chain reaction (PCR) of genomic DNA was performed using forward (5' - GGTGGTGCCCATCCTGGTCGAGC - 3') and reverse (5' - CCAGCATGCCTGCTATTGTCTTCCC - 3') primers (Invitrogen, Grand Island, NY) which were designed based on the EGFP coding sequence. Final PCR product was electrophoretically separated on a $5 \%$ agarose gel and visualized with ethidium bromide. All CNPase ${ }^{+}$pups were weaned at 3 weeks and housed with CNPase ${ }^{+}$littermates and other age/sex matched CNPase ${ }^{+}$ litters for at least 5 weeks ( 8 weeks of age) before being included in the study.

Cuprizone Treatment. Age and weight matched male and female mice were fed ad libitum a diet of $0.2 \%(\mathrm{w} / \mathrm{w})$ oxalic bis-cyclohexylidenehydrazide (cuprizone; 
Sigma-Aldrich, Milwaukee, WI) mixed into normal chow (Harlan Teklad, Madison, WI) beginning at 8 weeks of age. CNPase ${ }^{+}$mice were distributed into 6 treatment groups as follows: 1 and 2 (no cuprizone at 8 and 20 weeks of age); 3 and 4 ( 3 and 6 weeks of cuprizone diet); 5 and 6 ( 6 weeks of cuprizone diet followed by 3 and 6 weeks recovery on normal chow). Two control groups were included to account for any age related differences in miRNA expression as the length of the cuprizone treatment regimen is considerable. Any miRNAs identified as being significantly different between the 8 week and 20 week old control groups were ruled out for further consideration as they may play a role in aging. For all other comparisons between experimental and control cuprizone groups, the control used was the 8 week group.

Tissue Processing. Mice were sacrificed with an overdose of $2.0 \%$ avertin $(2,2,2-$ tribromoethanol) in sterile saline administered i.p. prior to transcardial perfusion with cold phosphate buffered saline (PBS) until the liver was clear and subsequent fixation with cold 4\% paraformaldehyde (PFA). Following fixation, brains were removed and post fixed for 24 hours in 4\% PFA. Cryoprotection was performed by immersion into a 30\% sucrose solution for at least 72 hours at 4 ${ }^{\circ} \mathrm{C}$. Brains were blocked in tissue freezing medium (Triangle Biomedical Sciences, Durham, NC) and frozen at $-20^{\circ} \mathrm{C}$ prior to coronal sectioning with a Leica cryostat (Buffalo Grove, IL) at $30 \mu \mathrm{m}$. Tissue sections were transferred to gelatin-coated slides and stored at $-20^{\circ} \mathrm{C}$. 
IHC. To distinguish CNPase ${ }^{+}$expressing cells from neurons and other glia, sections were stained with primary antibodies against glial fibrillary acidic protein (GFAP) to identify astrocytes (Fig. 9B), neurofilament medium (NF-M) to identify neurons (Fig. 9C) and CNPase to identify oligodendroglial lineage cells (Fig. 9A). Staining protocols were as follows: tissue sections were blocked with 5\% BSA, $10 \%$ normal donkey serum (NDS), and $0.1 \%$ Triton-X-100 in Tris-buffered saline (TBS) for $1 \mathrm{~h}$ at room temperature. Primary polyclonal antibodies NF-M (rabbit, 1:250, Millipore; Billerica, MA), GFAP (rabbit, 1:500, Dako; Carpinteria, CA), CNPase (rabbit, 1:250, Sigma) were incubated for $24 \mathrm{~h}$ at $4{ }^{\circ} \mathrm{C}$ with $5 \%$ BSA, $5 \%$ NDS, and $0.1 \%$ Triton-X-100 in TBS. Sections were next washed at room temperature $(\times 3)$ with TBS and incubated with fluorescein (FITC; 1:200) or rhodamine (TRITC; 1:200)-conjugated $\mathrm{F}\left(\mathrm{ab}^{\prime}\right)$ 2 secondary antibodies (donkey, Jackson ImmunoResearch; West Grove, PA) for $1 \mathrm{~h}$ at room temperature. Species-specific IgG isotype controls were used to account for any non-specific binding or other cellular protein interactions. Hoechst ${ }^{\mathrm{TM}}$ was used to identify nuclei. Fluorescent imaging was done on a Nikon Eclipse TE 300 inverted microscope with a spot CCD camera. Exposure times remained identical across all images. Endogenous CNPase-EGFP reporter expression was co-localized with polyclonal CNPase expression as confirmation that endogenous expression is sufficient to label oligodendroglia (data not shown). Callosal regions of interest (ROIs) were selected in serial cross sections by tracing the corpus callosum from midline to the lateral extent of corpus callosum $-2.00 \mathrm{~mm}$ behind bregma (area outlined and labeled as C.C.; Fig. 9D,E,F). Bilateral EGFP intensity within the 
selected $\mathrm{ROI}$ of $\mathrm{CNPase}^{+}$brains compared to CNPase ${ }^{-}$callosal EGFP intensity was used to estimate the relative decrease and return in CNPase expressing oligodendroglia during cuprizone de- and re-myelination, respectively (Fig. 9G). ROI selection and EGFP intensity quantification was done with Elements software (Nikon) and student t-tests were performed (Fig. 9G). Data are presented as mean \pm standard deviation ( $n=3$ / cuprizone treatment condition assessed).

Callosal Dissection and Tissue Dissociation. In total, 207 CNPase $^{+}$mice were used for miRNA microarray experiments. A sample from at least 2 wild type (WT) mice subject to the same dissection and digestion protocol served as a negative control to EGFP expression in fluorescence activated cells sorting (FACS) experiments. The corpus callosi of 10 to 15 mice within the same experimental group were pooled to yield an $n=1$. This was replicated in separate experiments to obtain an $n=3$. Mice were anesthetized with $2.0 \%$ avertin in sterile saline administered i.p. prior to transcardial perfusion with cold phosphate buffered saline (PBS). Following perfusion, brains were removed and the corpus callosum from bregma to $-2.5 \mathrm{~mm}$ bregma was dissected away from surrounding cortical and striatal tissue in cold HBSS lacking calcium and magnesium (Invitrogen,). Following dissection, corpus callosi were homogenized by hand using a razor blade in $1 \mathrm{~mL}$ fresh HBSS. Tissue homogenates were collected into a $15 \mathrm{~mL}$ conical tube and digested for 15 minutes at $37^{\circ} \mathrm{C}$ in enzyme mix 1 of the Neural Dissociation Kit (Miltenyi Biotec, Bergisch Gladbach, Germany). Following 
mechanical dissociation with a wide bore fire polished Pasteur pipette, samples were digested with enzyme mix 2 of the Neural Dissociation Kit for 10 minutes at $37^{\circ} \mathrm{C}$ with gentle agitation. Next, tissue was further mechanically dissociated with a medium and small bore fire polished Pasteur pipette, successively, sieved through a $40 \mu \mathrm{m}$ cell strainer (BD Falcon, Bedford, MA) and then incubated at $37^{\circ} \mathrm{C}$ for an additional 10 minutes prior to centrifugation with agitation. Single cell number was counted with a hemocytometer. The cell suspension was diluted 1:5 in cold HBSS with calcium and magnesium (Invitrogen) and centrifuged for 10 minutes at $300 \mathrm{~g}$. Cell pellets were re-suspended in $8.5 \mathrm{~mL}$ MACS buffer stock (Miltenyi Biotec) containing 5\% bovine serum albumin (BSA) and ??mg/mL insulin which was added to $5.7 \mathrm{~mL}$ of $90 \%$ percoll (Sigma) in 10x HBSS and centrifuged at $15,000 \mathrm{~g}$ for 15 minutes to remove myelin. Following centrifugation, myelin was aspirated from the top and the middle glial layer of cells was removed and centrifuged at $1200 \mathrm{~g}$ for 10 minutes to remove the percoll. Cells were washed twice and resuspended in a final volume of $0.5 \mathrm{~mL}$ MACS buffer containing BSA and insulin in preparation for FACS-based experiments.

FACS. Glial layer samples were subjected to FACS using a MoFlo system (DAKO, Ft. Collins, CO). Cells remained on ice throughout all sorting experiments. The samples were loaded and event flow was established. The triggering setting was reconfigured from cell size to fluorescence. Real time analysis of the pulse width versus forward scatter allowed for exclusion of 
doublets. Event triggering was based on the fluorescence of endogenous EGFP reporter or CD45-conjugated peridinin chlorophyll protein (perCP) fluorescence at an excitation of $488 \mathrm{~nm}$ and detection at $530 / 40 \mathrm{~nm}$ and $678 \mathrm{~nm}$, respectively. Callosal tissue from WT mice was used as a negative control for EGFP expression and the $\lg G$ isoltype-conjugated perCP control to established background perCP expression and the gating parameters. A bright cell population (gate R3; Fig. 10A) was consistently present above background in all samples and was $52.34 \% \pm 7.45 \%$ ( \pm standard deviation) of total sorted events (gate R1; Fig. 10A). To assess the presence of any myeloid lineage cells in sorted populations, un-sorted cell suspensions were incubated with either an antibody against common leukocyte surface antigen CD45 conjugated to perCP (1:100; BD Biosciences, San Jose, CA) or a perCP-conjugated IgG isotype control antibody (1:100; BD Biosciences) for 30 minutes at $37^{\circ} \mathrm{C}$ in culture media and washed $1 \mathrm{x}$ with DPBS prior to final re-suspension in cold DPBS for sorting.

Microarray. Following sorting, all samples were maintained at $4{ }^{\circ} \mathrm{C}$ throughout. Sorted cells were centrifuged at $300 \mathrm{~g}$ for 10 minutes and supernatant was aspirated. Total RNA was extracted from cell pellets immediately following sorting using the mirVana ${ }^{\mathrm{TM}}$ miRNA Isolation Kit (Invotrogen) as per the manufacturers protocol for cells in suspension. Once eluted from the column, total RNA was immediately frozen and stored at $-80^{\circ} \mathrm{C}$. Once all microarray samples were collected ( $n=3$ / group), samples were shipped on dry ice to Exiqon (Woburn, MA) where a single color genome wide miRNA expression 
profile analysis was performed using miRCURY LNA ${ }^{\mathrm{TM}}$ miRNA Arrays. Exiqon performed all miRNA quality control, extraction, enrichment and normalization and data were received as expression values above background. Principal component analysis (PCA) was performed with the statistics package $R$ ([public domain] http://www.r-project.org). Differentially expressed miRNAs were identified statistically by group ANOVAs performed across cuprizone treatment conditions with Benjamini Hochberg correction for false discovery rate (FDR; Pawitan et al., 2005). Unsupervised hierarchical clustering was performed with Cluster 3.0 ([public domain] rana.lbl.gov/EisenSoftware.htm) using the average linkage method (Eisen et al., 1998).

qRT-PCR. All qRT-PCR experiments were performed with an ABI 7900HT realtime PCR instrument (Invitrogen). Gene amplification for all mRNA and miRNA experiments was performed with pre-designed primer assays (Qiagen, Valencia, CA) and 20x Taqman ${ }^{\circledR}$ miRNA primer assays (Invitrogen), respectively. RNA concentrations were estimated with a NanoDrop 5000 machine with RNA elution buffer as a blank. First strand synthesis of complimentary DNA (cDNA) was performed using the $\mathrm{RT}^{2}$ First Strand Kit and $\mathrm{RT}^{2} \mathrm{SYBR}^{\circledR}$ Green ROX Master Mix (both from Qiagen) was used for cDNA detection. miRNA reverse transcription (RT) was performed with the TaqMan ${ }^{\circledR}$ MicroRNA Reverse Transcription Kit (Invitrogen) in combination with 5x miRNA-specific RT-primers and qRT-PCR analysis was performed by adding miRNA-RT product to Taqman ${ }^{\circledR}$ universal PCR master mix (Invitrogen). Target and reference gene amplification was 
performed in separate tubes and run in triplicate. Mouse specific primer assays used in combination with $\mathrm{RT}^{2} \mathrm{SYBR}^{\circledR}$ Green ROX Master Mix are as follows: Oligodendrocyte transcription factor 2 (Olig2; PPM04752B), platelet-derived growth factor receptor alpha (PDGFRa; PPM03640D), proteolipid protein 1 (PLP1; PPM04717C) and glyceraldehyde-3-phosphate dehydrogenase (Gapdh; PPM02946E; all purchased as pre-designed primer sets from Qiagen) while custom Invitrogen primers were used to access gene expression levels for myelin basic protein (MBP; forward primer 5' - GCCCATTGGTACACACTAAC - 3'and reverse primer 5' - CTCGATTCAGTGACAGGAAC - 3'), glial fibrillary acidic protein (GFAP; forward primer 5' - TCCTGGAACAGCAAAACAAG - 3'and reverse primer 5' - CAGCCTCAGGTTGGTTTCAT - 3'), microtubule associated protein 2 (Map 2; forward primer 5' - GAACTGACTCTCTCTGGATCTG - 3'and reverse primer 5' - ACCTTCAGCCAAACTGAGC - 3'). $\operatorname{Taqman}^{\circledR} 20 x$ miRNA primer assays were as follows: mmu-miR-338-5', mmu-miR-297c-5', U6 small nucleolar RNA (U6 snRNA) and small nucleolar RNA 135 (snoRNA 135; all purchased from Invitrogen). RNA levels were quantified using the delta-delta CT $(\triangle \Delta C T)$ method. Expression values from triplicate runs were normalized to reference gene triplicate values (Gapdh for SYBR ${ }^{\circledR}$ Green ROX based experiments and U6 snRNA or snoRNA135 for miRNA based experiments). Transcript levels are reported as fold change compared to normalized calibration sample gene expression. A one-way analysis of variance (ANOVA) was used to assess significance and data are presented as mean \pm standard deviation $(n=4 /$ group). 
OP Isolation from Mouse Pup Cortex. The cortices of both male and female postnatal day 5 (P5) C57BI/6 mouse pup cortices were dissected away from whole brain as previously described (Dincman et al., 2012). Briefly, tissue was dissociated using the Neural Tissue Dissociation Kit (Miltenyi Biotec) according to the manufacturer's protocol. Following dissociation, the entire cell suspension was incubated for 7 minutes at $4{ }^{\circ} \mathrm{C}$ with a mouse $\mathrm{O} 4$ hybridoma supernatant containing antibodies which recognize the $\mathrm{O} 4$ sulfatide. Following incubation, cells were washed and incubated with rat anti-mouse IgM magnetic beads (10\% diluted in MACS buffer; Miltenyi Biotec,) which bind only to $\mathrm{O}^{+}$cells within the suspension. Following two washes, the magnetically bound cell are maintained in the column while all $\mathrm{O}^{-}$cells are eluted and discarded. Between 8,000 and $12,000 \mathrm{cells} / \mathrm{cm}^{2}$ were finally eluted into a pre-equilibrated $10 \mathrm{~cm}$ tissue culture dish coated with poly-D-lysine (PDL) and laminin (both from Sigma) containing mouse OPC-A (mOPC-A) medium which was prepared as follows: Add $2.1 \mathrm{~g} / \mathrm{L}$ $\mathrm{NaHCO}_{3}$ (Sigma) to DMEM-F12 lacking HEPES (Invitrogen) and supplement with $1 \times$ N2, 2 x B27, 1\% penicillin/streptomycin (all from Invitrogen), BSA (0.01\%, Sigma), 40 ng/ml FGF2 (Millipore, Billerica, MA), and 20 ng/ml PDGFaa (Sigma). Average OP yield was $8-10 \times 10^{6}$ cells/brain with $85-95 \%$ viability. Following isolation, cells were incubated at $37^{\circ} \mathrm{C}$ with $5 \% \mathrm{CO}_{2}$.

Cell Culture. Following final cell elution into the pre-equilibrated $10 \mathrm{~cm}$ tissue culture dish (passage 0; P0), cells were maintained in mOPCa medium for 24 hours and incubated at $37^{\circ} \mathrm{C}$ with $5 \% \mathrm{CO}_{2}$. To assess the role of miRNAs 
identified via microarray in differentiating primary OPs, cells were exposed following the 24 hour post-elution incubation period to either mOPCa medium to drive proliferation or mOPC differentiation medium for 72 hours. mOPC differentiation medium was prepared as follows: Add $2.1 \mathrm{~g} / \mathrm{L} \mathrm{NaHCO}_{3}$ (Sigma) to DMEM-F12 lacking HEPES (Invitrogen) and supplement with 1 x N2, 2 × B27, 1\% penicillin/streptomycin (all from Invitrogen), BSA (0.01\%) and T3 (40 ng/mL; both from Sigma). Following experimental condition assignment and the 72 hour differentiation or proliferation period, total RNA was collected from each plate. Total RNA was also collected immediately following the 24 hour post-elution time-point from a subset of plates to serve as a calibration control for qRT-PCR experiments. A single plate is considered $\mathrm{n}=1$ and each condition (72 hours mOPCa medium, 72 hours mOPC differentiation medium or the 24 hour mOPCa medium calibration sample) was replicated to achieve $n=4$ for qRT-PCR based experiments and $\mathrm{n}=2$ for immunocytochemical (ICC) assessment of oligodendroglial differentiation markers.

Multiple miRNAs identified via microarray were screened in vitro with qRT-PCR following primary OP proliferation or differentiation. One miRNA was found to be consistently upregulated during primary OP differentiation versus OP proliferation; miR-297c. Therefore, to further assess the regulatory role for miR297c, gain and loss of function assays were performed by transfecting miRNA mimics or inhibitors (Invitrogen). Due to the high sensitivity of primary mOPCs to transfection reagent toxicity, miRNA mimic/inhibitor transfection was unable to 
achieve an acceptable transfection efficiency using non-toxic transfection reagent concentrations. Therefore, miRNA mimic/inhibitor gain and loss assays were performed using mouse embryonic fibroblasts (MEFs; SCRC-1040; ATCC, Manassas, VA) as they are more tolerant to higher transfection reagent concentrations. P0 MEFs were expanded for at least 2 passages in $10 \mathrm{~cm}$ tissue culture dishes lacking a substrate coating in MEF expansion medium which was prepared as follows: Add $3.7 \mathrm{~g} / \mathrm{L} \mathrm{NaHCO}_{3}$ (Sigma) to DMEM with high glucose and supplemented with $1 \%$ non-essential amino acids, $1 \%$ sodium pyruvate, 0.1 $\mathrm{mM} \beta$-mercaptoethanol ( $\beta-\mathrm{ME}), 1 \%$ penicillin/streptomycin (all from Invitrogen) and $10 \%$ fetal bovine serum (FBS; Atlanta Biologicals, Flowery Branch, GA) and maintained at $37^{\circ} \mathrm{C}$ with $5 \% \mathrm{CO}_{2}$. MEFs were then allowed to incubate for 24 hours prior to transfection. For miRNA mimic/inhibitor transfection experiments, MEFs were plated at 20,000 cells $/ \mathrm{cm}^{2}$ in MEF transfection medium which is identical to MEF expansion medium but containing 5\% FBS rather than 10\%. Mimic/inhibitor transfection was performed by first incubating RNAiMAX (Invitrogen) with either miR-297c mimic, miR-297c inhibitor, negative control mimic, negative control inhibitor or transfection reagent alone (all from Invitrogen) for 30 minutes in serum-free Opti-MEM ${ }^{\circledR}$ (Invitrogen) at $37^{\circ} \mathrm{C}$. Mimics/inhibitors were added to the MEF cultures already containing MEF transfection medium and allowed to incubate overnight. Transfection efficiencies were established to be $41.53 \% \pm 15.67 \%$ ( \pm standard deviation) with pilot experiments transfecting either $\mathrm{FAM}^{\top \mathrm{M}}$ Dye-labeled pre- or anti-miR controls (Invitrogen) into MEFs thus allowing FACS-based assessment of $\mathrm{FAM}^{+}$events relative to total events (Fig. 
11). An Opti-MEM ${ }^{\circledast}$ alone condition was included to assess any effects from the transfection reagent alone. The following morning, a full media change with MEF transfection media was performed on all plates prior to being returned to the incubator for 24 additional hours. For cell cycle analysis, MEFs were collected from plates and fixed with ice-cold $70 \%$ ethanol $(\mathrm{EtOH})$ for at least 48 hours at $20^{\circ} \mathrm{C}$. A single plate is considered $\mathrm{n}=1$ and each condition was replicated to achieve $\mathrm{n}=4$ for qRT-PCR experiments.

ICC. Cells were washed in DPBS and incubated with the appropriate hybridoma supernatant (A2B5, O4 or O1) diluted to $70 \% \mathrm{v} / \mathrm{v}$ in $20 \%$ DPBS, and $10 \%$ NDS for 45 minutes at $4{ }^{\circ} \mathrm{C}$. Following washes (x3) with DPBS and fixed with $4 \%$ PFA at room temperature for 10 minutes. Cells were incubated overnight with additional primary polyclonal antibodies NG2 (rabbit, 1:500, Millipore, Billerica, MA), $\beta$ III-tubulin (rabbit, 1:2000, Covance, Princeton, NJ), MBP (rat, 1:100, Millipore), GFAP (rabbit, 1:500, Dako, Glostrup, Denmark) and Map2 (rabbit, 1:100, Sigma) at $4{ }^{\circ} \mathrm{C}$ diluted in blocking solution containing $10 \%$ NDS, $0.5 \%$ BSA and $0.1 \%$ Triton X-100 in DPBS. The following morning, cells were washed (x3) with TBS and incubated with fluorescein (FITC; 1:200) or rhodamine (TRITC; 1:200)-conjugated $F\left(a b^{\prime}\right) 2$ secondary antibodies (donkey, Jackson ImmunoResearch; West Grove, PA) for $1 \mathrm{~h}$ at room temperature. Speciesspecific IgG isotype controls were used to account for any non-specific binding or other cellular protein interactions. Hoechst ${ }^{\mathrm{TM}}$ was used to identify nuclei. Photomicrographs were captured using a Nikon TiE 300 inverted microscope 
with a DXM-1200C coded digital camera and NIS Elements software (Nikon, Melville, NY). Counts of monolayered cultured cells were performed in 5 randomly chosen fields per culture dish well and percentages of $\mathrm{A} 2 \mathrm{~B} 5^{+} / \mathrm{Hoechst}^{+}$, $\mathrm{O} 4+/ \mathrm{Hoechst}_{+}, \mathrm{O}^{+} / \mathrm{Hoechst}^{+}$or $\mathrm{GFAP}^{+} / \mathrm{Hoechst}^{+}$cells per total $\mathrm{Hoechst}^{+}$nuclei were used for quantification. A one-way ANOVA was performed and data are presented as a mean percentage \pm standard deviation.

Cell Cycle Analysis. Following transfection, cells were washed (x2) with DPBS to remove serum containing media and harvested by trypsinization for 3 minutes at $37^{\circ} \mathrm{C}$ after which they were collected and diluted with MEF media containing $10 \%$ FBS and centrifuged at $300 \times \mathrm{g}$ for 5 minutes. Following centrifugation, cell pellets were washed (x2) with DPBS to remove media and trypsin. Finally, the supernatant was aspirated and ice cold $70 \%$ ethanol $(\mathrm{EtOH})$ was added to the cell pellets with gentle trituration and allowed to fix for at least 48 hours. Following fixation, cells were centrifuged at $300 \times \mathrm{g}$ for 5 minutes and washed (x2) with DPBS. Cells were then stained overnight with the DNA intercalator propidium iodide $(\mathrm{PI})$ to assess DNA content. The following day, cells were analyzed with a 3-channel FACSCaliber flow cytometer (BD Biosciences Immunocytometry Systems, San Jose, CA). Data were exported as FCS files and further analyzed using Flowjo software (treestar.com). The $G_{1} / G_{0}$ and $G_{2} / M$ histogram peaks were fit to the Watson Pragmatic Model (Watson et al., 1987) and the $G_{1} / G_{0}$, S-phase and $G_{2} / M$ frequencies were quantified. Student t-tests were performed between mimic/inhibitor transfection group and transfection 
control groups. A one-way ANOVA was performed and data are presented as mean \pm standard deviation.

\section{Results}

CNP-EGFP ${ }^{+}$cells sorted from callosal tissue display mRNA expression profiles highly consistent with that of OPs and not mature OLs or other CNS cell types.

Animal weights were monitored weekly throughout the course of the experiment and any outliers were removed from the study. Outliers are defined as being \pm 2 standard deviations from the mean weight of all age matched mice. IHC confirmed that CNP-EGFP expression was specific to oligodendroglia and not other CNS cell types (Fig. 9A) and that cuprizone-induced de- and re-myelination within the corpus callosum was accompanied by a reduction and return of callosal CNP-EGFP expression, respectively (Fig. 9B,C).

To confirm the lineage and developmental stage of $\mathrm{CNPase}^{+}$cells, RNA isolated from sorted cells was screened with qRT-PCR to assess the expression levels of genes differentially expressed in OPs (Olig2 and PDFGRa; Fig. 10B), OLs (Olig2 and MBP; Fig. 10B), astrocytes (GFAP; Fig. 10B), neurons (Map2; Fig. 10B) and myeloid lineage cells (CD45; Fig. 12B) relative to RNA isolated from an equal post-dissection volume of homogenized whole callosal tissue which contains RNA from all CNS cell types (Fig. 10B). Sorted naïve cells displayed robust 
expression levels of Olig2 and PDGFRa, both genes highly expressed in oligodendrocytic progenitors (Fig. 10B). Although Olig2 is expressed in both OPs and mature myelinating OLs, PLP2 and MBP (two genes specifically expressed in myelinating OLs) were not highly expressed in sorted cells suggesting an absence of mature OLs from sorted populations (Fig. 10B). This is not surprising as the dissection, digestion, and dissociation protocol combined with myelin layer removal is likely to have eliminated mature OLs from sorted populations. Other genes representative of non-oligodendroglial CNS cell types displayed low expression levels compared to whole tissue suggesting that there is little to no contamination from astrocytes (GFAP; Fig. 10B) or neurons (Map2; Fig. 10B) in sorted populations. All the data presented above has been in reference to naïve callosal OPs. Sorted callosal OPs following 6 weeks of cuprizone treatment displayed similar gene expression profiles for Olig2, PDGFRa, MBP, GFAP, Map2 and CD45, but PLP1 expression was significantly up-regulated in the 6 week cuprizone group compared to the no cuprizone group (Fig. 10B). Using 6 weeks on cuprizone RNA, no $\mathrm{CD} 45^{+}$cells were present when sorting with the IgG-perCP isotype control (gate R4; Fig. 12A) but a negligible number of $\mathrm{CD}_{4}{ }^{+} / \mathrm{CNPase}^{+}$cells were identified using the CD45-perCP antibody representing $<0.2 \%$ of sorted CNPase ${ }^{+}$events (gate R4; Fig. 12B).

Sfmbt2 cluster miRNAs were identified via microarray and share similar expression profiles during cuprizone-induced de- and re-myelination. 
Bioanalyzer (Agilent Technologies, Santa Clara, CA) results provided by Exiqon revealed acceptable RNA quality for all samples. PCA demonstrated clustering of samples with other replicate samples from a given cuprizone treatment condition and segregation of clustered samples between cuprizone treatment conditions (data not shown). Out of 1,215 mouse miRNAs assayed in the microarray, 439 miRNAs were identified as being present above background in each sample (regardless of cuprizone treatment condition) demonstrating consistency across cuprizone conditions but not accounting for abundance. Using group ANOVAs corrected for FDR, 237 miRNAs out of 439 changed significantly across cuprizone treatment conditions. A list was comprised a priori to assess if miRNAs identified in the microarray were consistent with oligodendroglial-derived or white matter-derived miRNAs previously identified in the literature (Fig. 13). There was no restrictive criterion to include a miRNA on the list in that it only needed to be loosely associated with oligodendroglia considering miRNAs identified in while white matter tissue were also included (Fig. 13). Overall, 38 miRNAs were included with sources ranging from human and rodent-derived oligodendroglia to human MS lesion tissue to human or rodent-derived Schwann cells (Fig. 13). Of the 38 miRNAs listed in Fig. 13, 34 (89\%) were also identified via microarray with 29 of the 34 miRNAs (85\%) identified as changing significantly across cuprizone treatment conditions as per the group ANOVA (Fig. 13).

No differences were identified between the 8 week and the 20 week no cuprizone groups. OP miRNA expression profiles are dynamically regulated during 
cuprizone-induced demyelination ( 3 and 6 weeks on cuprizone) and remyelination ( 3 and 6 weeks off cuprizone; Fig. 14A). Moreover, despite the fact that the callosi of multiple age matched mice (both male and female) were pooled together to yield sufficient RNA quantities, the miRNA expression values across N's of a particular cuprizone treatment condition were highly reproducible (Fig. 14A). Regression analyses of the miRNA expression levels for all miRNAs between 2 samples of a given treatment condition were performed for all possible sample comparison combinations and revealed that all 3 samples in the no cuprizone, aged cuprizone, 3 weeks and 6 weeks on cuprizone groups had an Rsquared $\left(R^{2}\right)$ value of $>0.95, p<0.001$ (data not shown). This demonstrates that expression levels across samples within a particular group were highly consistent for the control, 3 and 6 weeks on cuprizone groups. Regression analyses of the 3 week and 6 week off cuprizone groups revealed that one of the 3 samples in each group displayed $R^{2}$ values of 0.59 and 0.80 , respectively, while the other 2 remaining samples in each group displayed $R^{2}$ values of $>0.97, p<0.001$. Additionally, the 2 outlier samples were determined by principle component analysis to not cluster with the other respective samples in their group. Therefore, these samples were removed from statistical analysis. Mean miRNA expression levels analyzed across cuprizone treatment conditions were ordered by corrected $\mathrm{P}$ value. Over half of the top 25 most significant miRNAs were members of the Sfmbt2 miRNA cluster, a recently identified group miRNAs located within $<10 \mathrm{~kb}$ of each other on intron 10 or 11 of the Sfmbt2 host gene (Wang et al., 2011; Fig. 14A). Moreover, hierarchical clustering demonstrated 
that 52 of $53(98 \%)$ Sfmbt2 cluster miRNAs clearly segregated together into 3 primary clusters representing 53\% (cluster A), 42\% (cluster B) and 83\% (cluster C) of the miRNAs in each respective cluster (data not shown). Furthermore, clusters A and B were associated by a primary node and all 3 clusters were associated by a secondary node (data not shown). Fold change relative to the no cuprizone group was assessed for 3 and 6 weeks on and 3 and 6 weeks off cuprizone revealing that nearly all Sfmbt2 cluster miRNAs share a similar expression profile across cuprizone treatment conditions (Fig. 14B). In total, 53 Sfmbt2 cluster miRNAs were identified in the microarray and each were determined to change significantly across cuprizone treatment conditions (group ANOVA corrected for FDR). All but one Sfmbt2 cluster miRNA (miR-466h) displayed a profile highlighted by a down-regulation of Sfmbt2 miRNAs at the 6 week on cuprizone time-point (Fig. 14B). Post-hoc t-tests of Sfmbt2 cluster miRNAs revealed that the decrease in expression observed at 6 weeks was significant for each miRNA analyzed relative to no cuprizone (data not shown).

Sfmbt2 cluster member miR-297c is up-regulated following differentiation of primary mouse OPs in vitro

24 hours post-MACS isolation, $93.94 \% \pm 6.84 \%$ of total Hoechst $^{+}$nuclei were also $\mathrm{A}_{2} \mathrm{~B}^{+}$and $83.24 \% \pm 9.35 \%$ were $\mathrm{O}^{+}{ }^{+} \pm$standard deviation; Fig. $15 \mathrm{~A}, \mathrm{C}$ ). Following 72 hours of differentiation with mitogen removal and T3 addition to the culture medium, process elongation and arborization were accompanied by a 
reduced percentage of $\mathrm{A} 2 \mathrm{~B} 5^{+}$to $14.87 \% \pm 7.77 \%$ and $\mathrm{O}^{+}$cells $46.23 \% \pm 7.77 \%$ relative to total Hoechst ${ }^{+}$nuclei (Fig. 15C). Moreover, $\mathrm{O}^{+}$cells which were not present 24 hours post-MACS was identified following 72 hours differentiation constituting $41.88 \% \pm 18.64 \%$ of total Hoechst $^{+}$nuclei (Fig. 15B,C). Antibodies raised against GFAP, an intermediate astrocyte filament, demonstrated GFAP ${ }^{+}$ immunoreactivity 24 hours post-MACS or following 72 hours proliferation to be $1.54 \% \pm 0.05 \%$ of total Hoechst ${ }^{+}$nuclei (Fig. 15A,C). Following 72 hours of differentiation, GFAP expression increased to $27.50 \% \pm 8.83 \% \%$ of total Hoechst $^{+}$nuclei (Fig. 15B,C). This suggests that along the course of differentiation, approximately a quarter of the primary mouse OPs magnetically sorted with the $\mathrm{O} 4$ sulfatide begin to follow along an astrocytic lineage rather than maintain an exclusive oligodendrocytic lineage. This is typical of cultured mouse OPs considering their bi-potentiality.

The expression profiles of multiple Sfmbt2 miRNAs as well as other miRNAs identified in the microarray were assessed during the differentiation of primary mouse OPs isolated from P5 mouse pup cortical tissue. The specific Sfmbt2 cluster member miRNAs screened was determined based on either novelty, anomalous microarray expression profile or a target prediction profiles with high prediction scores for oligodendroglial transcripts. Although the microarray revealed a significant change as per the group ANOVA for miR-338-5', post-hoc t-tests revealed that there was no change in miR-338-5' expression at 3 weeks and 6 weeks on cuprizone relative to no cuprizone (data not shown). Our a priori 
assumption was that miR-338-5' would be up-regulated at 6 weeks on cuprizone considering that qTR-PCR experiments revealed increased PLP1 mRNA expression at 6 weeks on cuprizone compared to no cuprizone (Fig. 10B) but this was not observed. However, the lack of an increase in miR-338-5' expression at 6 weeks on cuprizone could be explained the possibility that sorted populations display an expression profile consistent with a progenitor phenotype (Fig. 10B) and miR-338-5' was shown to increase concomitant with the expression of mature OL myelination genes (Dugas et al., 2010; Hu et al., 2010). Therefore, terminally committed oligodendroglia displaying increased miR-338-5' were likely eliminated during the isolation protocol and do not contribute the RNA isolated from sorted cells. Moreover, although there is a significant increase observed in PLP1 mRNA at 6 weeks post-cuprizone, it is likely that in addition to proliferating OPs, sorted populations also contained terminally committed oligodendroglia in the very initial stages of remyelination. In this pool of cells, PLP protein production and myelination has not yet completed making them less susceptible to percoll removal thus representing a higher proportion of sorted populations thereby accounting for the increase on PLP1 expression. Although miR-338-5' displayed no change in expression via the microarray, we still view it as an acceptable positive control to differentiation in vitro as it has been previously identified by Dugas et al. (2010) and Hu et al. (2010) to increase concomitant with $\mathrm{OL}$ differentiation. Consistent with what has been previously described, miR338-5' was significantly increased in primary mouse OPs following 72 hours of differentiation compared to 72 hours of proliferation (Fig. 15D). Of all Sfmbt2 
cluster miRNAs assayed, only miR-297c was significant, consistently displaying expression levels of 3 fold or higher in differentiating mouse OPs relative to proliferating mouse OPs (Fig. 15D).

miR-297c regulates S-phase transition in cycling MEFs

Three separate target prediction algorithms (miRDB, miRanda and TargetScanMouse) each predicted miR-297c to target the cyclin T2 (CCNT2) transcript with a high reliability score. Considering that CCNT2 has recently been described as a regulator of $\mathrm{G}_{1}$ to $\mathrm{S}$-phase transition via its interaction with cyclin dependent kinase 9 (CDK9) in P-TEFb complex formation (Fig. 16A; Peng et al., 1998; Price, 2000), miR-297c mimics and inhibitors were transfected into cycling MEFs to assess a role for miR-297c in $\mathrm{G}_{1}$ to $\mathrm{S}$-phase transition using cell cycle analysis (Fig. 16B). Fig. 16 is a representative schematic of $P$-TEFb-induced $G_{1}$ to S-phase transition (Fig. 16A) and its possible miR-297c-induced repression (Fig. 16B). Cell cycle analysis revealed that 48 hours following miR-297c or control transfection, MEFs transfected with miR-297c displayed a significant increase in $G_{1} / G_{0}$ frequencies (Fig. 17A) and a significant reduction in S-phase frequencies (Fig. 17B).

\section{Discussion}


The mRNA expression profile of sorted cells from naïve adult mice was highly consistent with that of an OP profile (Fig. 10B). Both Olig2 and PDGFRa transcripts were present within sorted populations in abundance. Olig2 is not considered here as a genetic identifier of OPs per se, but rather as a general genetic identifier of oligodendroglial cells at any developmental stage. Although the presence of Olig2 mRNA in sorted populations was robust, this does not distinguish between terminally committed OLs versus mitotically competent OPs. However, considering that MBP and PLP1 mRNA expression was minimal, this suggests that sorted populations were of a less mature phenotype. Furthermore, PDGFRa mRNA expression in sorted populations was robust which again suggests an immature phenotype. PDGFRa (CD140a) expression has been demonstrated to be specific to OPs in both rodent (Pringle et al., 1992; Ellison and de Vellis, 1994) and humans (Sim et al., 2006). Using CD140a based isolation of OPs from human tissue, PDGFRa expressing cells were shown to represent a subpopulation of OPs which favor an oligodendrocytic rather than an astrocytic fate (Sim et al., 2011). Therefore, the CNPase ${ }^{+}$sorted OPs we report here most likely were destined to follow down an oligodendroglial lineage and thus mRNA expression profiles are representative of OPs already committed to become oligodendrocytes rather than truly bipotential OPs. Although other CNS cell types are also present in the sorted population, low GFAP and Map2 expression suggests that their contribution to total RNA pools was minimal and that their numbers in sorted populations were few making the purity of sorted OPs more than acceptable for purposes described here. 
The significant increase in PLP1 expression following 6 weeks on cuprizone compared to no cuprizone (Fig. 10B) is not surprising as PLP1 mRNA has been identified in developing and adult mouse NG2 ${ }^{+}$OPs (Ye et al., 2003). Within the 6 week compared to the no cuprizone corpus callosum, there are likely higher proportions of OPs which have not only terminally committed to an oligodendroglial fate but are in or nearing the pre-myelinating stage and therefore do not yet express mature myelin. Therefore, unlike their mature myelinating counterparts, these pre-myelinating oligodendroglia would be less sensitive to disruption from either digestion or dissociation increasing their proportion in sorted populations thus inflating PLP1 transcript levels in RNA from sorted populations.

Inflammation within the corpus callosum is observed following 6 weeks of cuprizone (Buschmann et al., 2012). Although CNPase is enriched within oligodendroglia, it has also been suggested to be present in reactive microglia (Wu et al., 2006). Although CNPase ${ }^{+} / \mathrm{CD} 45^{+}$cells were present in sorted populations, their contribution was negligible, $<0.20 \%$ of total sorted events. Interestingly, there was also a small subset of CNPase ${ }^{-} / \mathrm{CD} 45^{+}$cells which are likely representative of systemic inflammatory cells that have infiltrated the demyelinated corpus callosum. 
The high percentage of miRNAs listed in Fig. 13 which are also expressed in the microarray strongly suggests that the miRNA expression profile of sorted populations is consistent with what has been previously described for oligodendroglial miRNAs. Sorted OPs displayed dynamically regulated miRNA expression profiles during cuprizone de- and re-myelination (Fig. 14A). Moreover, despite the fact that the callosi of multiple age matched mice (both male and female) were pooled together to yield sufficient RNA quantities, the miRNA expression values across Ns of a particular cuprizone treatment condition were highly reproducible (Fig. 14A). Included in the most significantly changed miRNAs are multiple members of the intrionic miRNA cluster located within the Sfmbt2 host gene (Fig. 14A,B). Nearly all Sfmbt2 cluster miRNAs identified in the microarray display a significant fold decrease (post-hoc t-test) in expression at 6 weeks on cuprizone. Sfmbt2 is a polycomb-group gene that when translated into protein, forms an interaction with the transcription factor ying yang 1 (YY1; Kuzmin et al., 2008), an important regulator of both OP (He et al., 2007) and Schwann cell differentiation (He et al., 2011). Sfmbt2 is also a host gene for a cluster of miRNAs located within $<10 \mathrm{~kb}$ of each other on intron 10 or 11 of the Sfmbt2 host gene (Wang et al., 2011). Certain Sfmbt2 cluster miRNAs have been identified in past studies to regulate cell cycle. Leone et al. (2011) recently demonstrated that Sfmbt2 cluster member mmu-miR-297a binds within the 3' untranslated region (UTR) of cyclin D2 (CCND2) transcripts thus negatively regulating CCND2 protein levels in thyroid cells in vitro. Human miR-297 was toxic to multiple glioblastoma cell lines with no effect on normal astrocytes 
suggesting that it is a negative regulator of tumor cell proliferation (Kefas et al., 2013). The Sfmbt2 cluster members mmu-miR-466h-5' and mmu-miR-669c were both identified to negatively regulate several anti-apoptotic genes in unison following glucose deprivation-induced oxidative stress (Druz et a., 2011). Using RISC-immunoprecipitation following miRNA transfection, others have demonstrated that mmu-miR-466d-3' binds to cyclin B1 (CCNB1) transcripts in the CCNB1 promoter (Huang et al., 2012). However, rather than negatively regulating CCNB1 translation, mmu-miR-466d-3' transfection induced CCNB1 protein expression while knockdown repressed CCNB1 protein levels (Huang et al., 2012). This paradoxical effect is not unprecedented as others have suggested a possible role for miRNAs in translation induction when binding to their targets within the 3' UTR of promoter regions (Vasudevan et al., 2007; Rusk, 2008), however this effect is still not completely understood and reported instances are rare proportional to the breadth of studies demonstrating a clear role for miRNAs in translational repression. Nearly all Sfmbt2 cluster miRNAs identified in the microarray displayed a robust decrease in expression at 6 weeks on cuprizone relative to no cuprizone (Fig. 14B). Using a customized qRT-PCR array screen, Hibbets et al., (2012) demonstrated that the microenvironment present within the corpus callosum at 6 weeks cuprizone contains highly elevated levels of the OP mitogen FGF2 (Hibbits et al., 2012). Taken together, the elevated callosal FGF2 levels at 6 weeks cuprizone and corresponding decreased microarray expression of a miRNA cluster known to negatively regulate proliferation at 6 weeks on cuprizone suggests that the Sfmbt2 miRNAs 
may normally repress proliferation but following an increase in mitogenic signaling via FGF2, these miRNAs are down-regulated to accommodate the translation of proteins required for cell cycle entry.

As validation that Sfmbt2 miRNAs regulate OPs, multiple Sfmbt2 miRNAs were screened with qRT-PCR following RNA isolation from primary mouse OPs allowed to differentiate or proliferate for 72 hours in vitro. One Sfmbt2 cluster member screened, mmu-miR-297c (miR-297c), was found to significantly increase concomitant with differentiation (Fig. 15D). Moreover, oligodendroglial enriched miRNA miR-338-5', previously shown to increase during oligodendroglial development in vivo (Zhao et al., 2010) and in vitro (Dugas et al., 2010; Zhao et al., 2010), was also significantly up-regulated in differentiating cells compared to proliferating OPs (Fig. 15D) thus serving as a positive control to mouse OP differentiation. Using the miRanda software, miRNA and target transcript alignment revealed that miR-297c targeted CCNT2 in at least 3 different miRNA binding regions on the CCNT2 transcript. One miR-297c binding region overlapped with the binding region of multiple other Sfmbt2 cluster miRNAs suggesting that many of the miRNAs within that cluster may not only regulate the same transcripts, but at the same binding region on the target transcript. Moreover, the oligodendroglial specific miRNAs miR-23a and miR338-5' which have been previously described to regulate oligodendroglial lineage cells, were both predicted by the miRDB algorithm to target the CCNT2 transcript. This suggests that miR-297c, miR-23a and miR-338-5' may play 
similar roles and share similar expression profile changes during differentiation and this is consistent with what we report here (Fig. 15D). CCNT2 is the regulatory subunit of P-TEFb, a complex formed by both CCNT1/2 proteins and cyclin dependent kinase 9 (CDK9; Kohoutek, 2009; Fig. 16A). Once formed, PTEFb can then interact with Bromodomain-containing protein 4 (BRD4) which recruits a now active P-TEFb to $G_{1}$ genes, a necessary step for $G_{1}$ to $S$-phase transition (Kohoutek, 2009; Fig. 16A). Therefore, increased miR-297c expression and subsequent repression of CCNT2 translation may prevent P-TEFb complex formation thus limiting S-phase transition resulting in $\mathrm{G}_{1} / \mathrm{G}_{0}$ cell cycle arrest (Fig. 16B). As miR-297c was described by 3 separate algorithms to target CCNT2 mRNA, we see this as a likely mechanism by which miR-297c regulates cell cycle entry (Fig. 16).

To assess the hypothesis presented in Fig. 16, MEFs were transfected with one of the following: miR-297c mimic, miR-297c inhibitor, negative control or transfection reagent alone and cell cycle analysis was performed with PI. The Sfmbt2 cluster member miR-669i was determined in pilot studies to not change during primary OP differentiation and was therefore included as a Sfmbt2 miRNA internal control (Fig. 17). The most likely cell cycle effect of reducing P-TEFb activation via repression of CCNT2 translation would be a reduction in S-phase cells and an increase in $\mathrm{G}_{1} / \mathrm{G}_{0}$ cells and this is consistent with data reported here (Fig. 17A,B). The fact that gain and loss of function assays for miR-297c were performed in MEFs rather than primary OPs or an oligodendroglial cell line does 
not preclude miR-297c from being considered as a regulator of other non-MEF mouse cells. Cell cycle genes are highly conserved across species and will certainly maintain high homology within different cells of the same organism. Therefore, whether or not miR-297c itself is normally present in MEFs is irrelevant as CCNT2 transcripts are equally as susceptible to regulation by exogenously applied miR-297c as they are to endogenous miRNAs.

In conclusion, Sfmbt2 miRNAs are dynamically regulated during cuprizoneinduced de- and re-myelination. Here, we report the first evidence of a role for the Sfmbt2 cluster member miR-297c in cell cycle control. This is but one of many studies which establish a role for miRNAs in cell cycle control (Wang and Blelloch, 2009; Chen and Hu, 2012; Malhas et al., 2010). However, this is the first documentation of miRNAs regulating cell cycle in oligodendroglia isolated from an in vivo source. Cell cycle progression in OPs is dependent on FGF2- and insulin-like growth factor 1 (IGF-1)-induced cyclin D1 protein production (Frederick and Wood, 2004; Frederick et al., 2007). Furthermore, promoting OP cell cycle exit by mitogen removal has been described as a potent inducer of OL differentiation (Casaccia-Bonnefil and Liu, 2003; He et al., 2007). Therefore, it is not surprising that miR-297c, which we have demonstrated here to increase concomitant with OP differentiation, also resulted in the cell cycle arrest when transfected into cycling cells. Together, these results suggest that strategies aimed at augmenting miR-297c expression in OP grafts prior to transplantation may favor oligodendrocytic differentiation thus promoting an optimal proportion of 
oligodendrocytes to astrocytes during remyelination. On the other hand, pharmacological strategies aimed at reducing miR-297c levels during endogenous remyelination may enhance the proliferative response of endogenous OP pools during remyelination ultimately providing more mature OLs and thus more remyelination. Further investigation is necessary to determine the effectiveness of miR-297c manipulation on remyelination in vivo.

\section{Acknowledgements}

We thank Darlene Burke for assistance with statistical analyses, Kariena Andres and Amberly Riegler for animal care, Allison Metz for cell culture assistance and Ashley Mullins for qRT-PCR and genotyping assistance. Supported by NS054708, P20 RR15576/GM103507, Norton Healthcare, Friends for Michael, Kentucky Spinal Cord and Head Injury Research Trust, and the Commonwealth of Kentucky Challenge for Excellence. 
Figure 9. Oligodendroglial CNPase-EGFP ${ }^{+}$reporter expression facilitates the quantification of cuprizone-induced de- and re-myelination. Endogenous CNPase-EGFP ${ }^{+}$reporter expression identifies oligodendroglial lineage cells within brain white matter (A) while not co-localizing with GFAP (B) or NF-M (C). Callosal CNPase-EGFP ${ }^{+}$reporter intensity was analyzed in selected ROls (D, E and F) following no cuprizone (D), 6 weeks on cuprizone (E) or 6 weeks off cuprizone $(F)$. Intensity levels above background were quantified $(G)$ revealing significant loss of callosal CNPase-EGFP ${ }^{+}$expression at 6 weeks on cuprizone ( $E$ and $G$ ) which returned by 6 weeks off cuprizone ( $F$ and $G$ ). Data in $G$ are presented as mean \pm standard deviation; ${ }^{*} p<0.05$, ${ }^{* *} p<0.001$. Scale bars in $A$, $\mathrm{D}, \mathrm{E}, \mathrm{F}$ and $\mathrm{B}, \mathrm{C}=1000 \mu \mathrm{m}$ and $100 \mu \mathrm{m}$, respectively. 

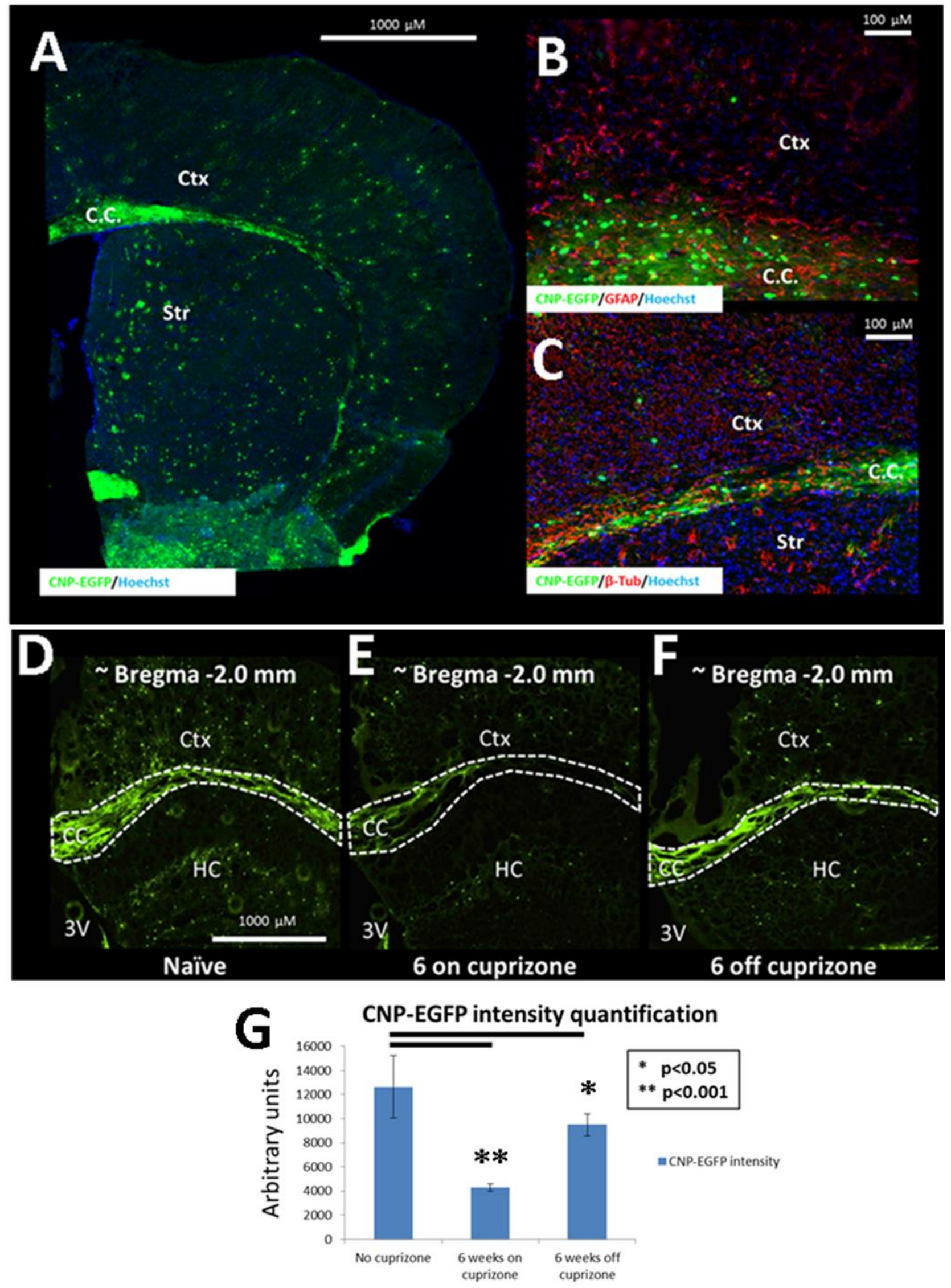

115 
Figure 10. FACS-based isolation of callosal CNPase-EGFP ${ }^{+}$yields a cell population with mRNA profiles highly consistent with OPs. A bright $\mathrm{EGFP}^{+}$cell population (gate R3; A) was consistently present above background in all samples and was $52.34 \% \pm 7.45 \%$ ( \pm standard deviation) of total sorted events (gate R1; A). qRT-PCR of sorted OPs demonstrated a high proportion Olig2 and PDGFRa transcripts relative to whole corpus callosum (B). Transcripts typically expressed in maturing oligodendroglia (MBP and PLP1) were not identified in sorted cell populations relative to whole corpus callosum (B). Map2 (B) and GFAP (B) transcripts were also not identified in sorted populations suggesting a highly oligodendroglial phenotype. Following 6 weeks of cuprizone, sorted OPs displayed a significant increase in PLP1 mRNA expression compared to no cuprizone (B). Data in B are presented as fold change \pm standard deviation; * $p<$ 0.05 . 
A

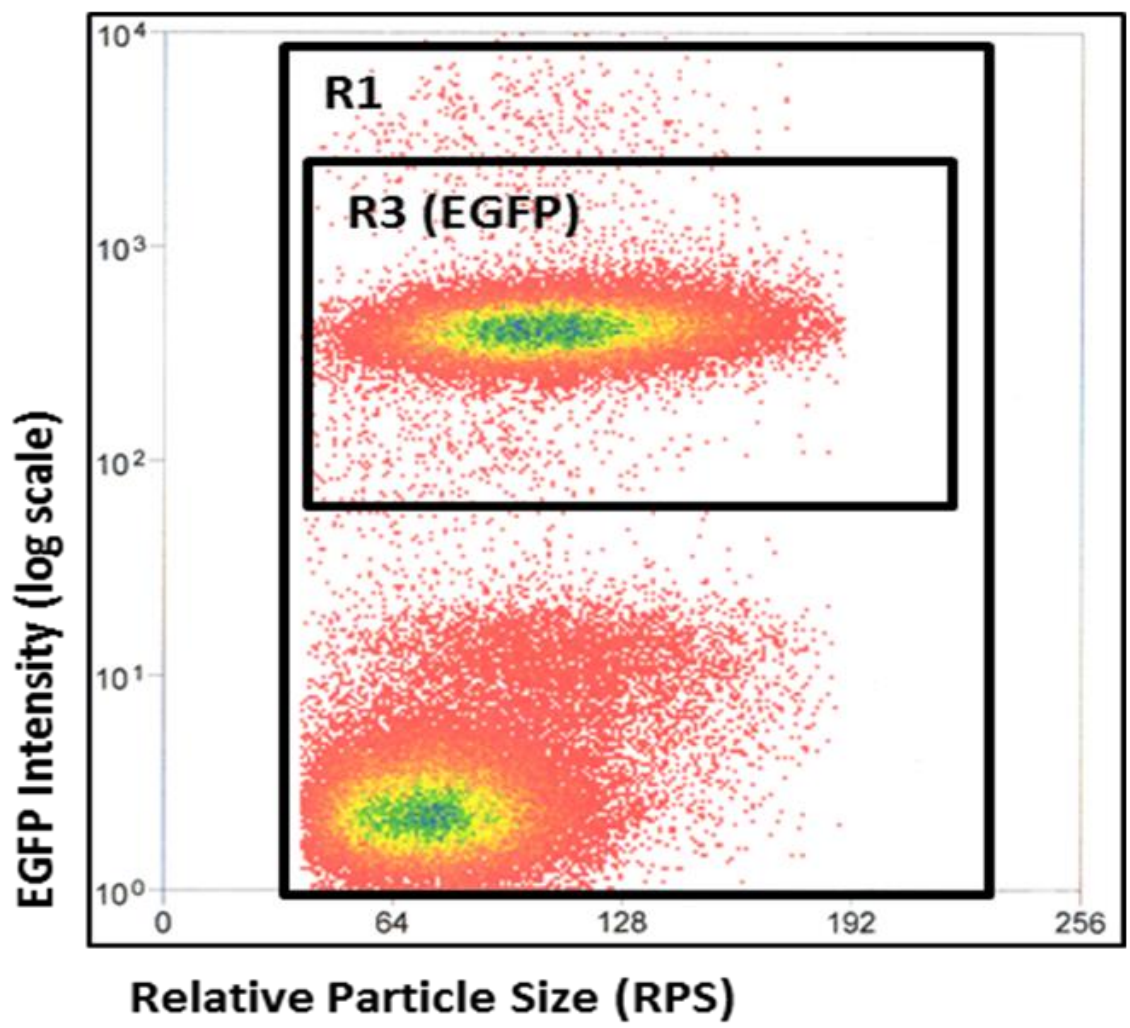

B

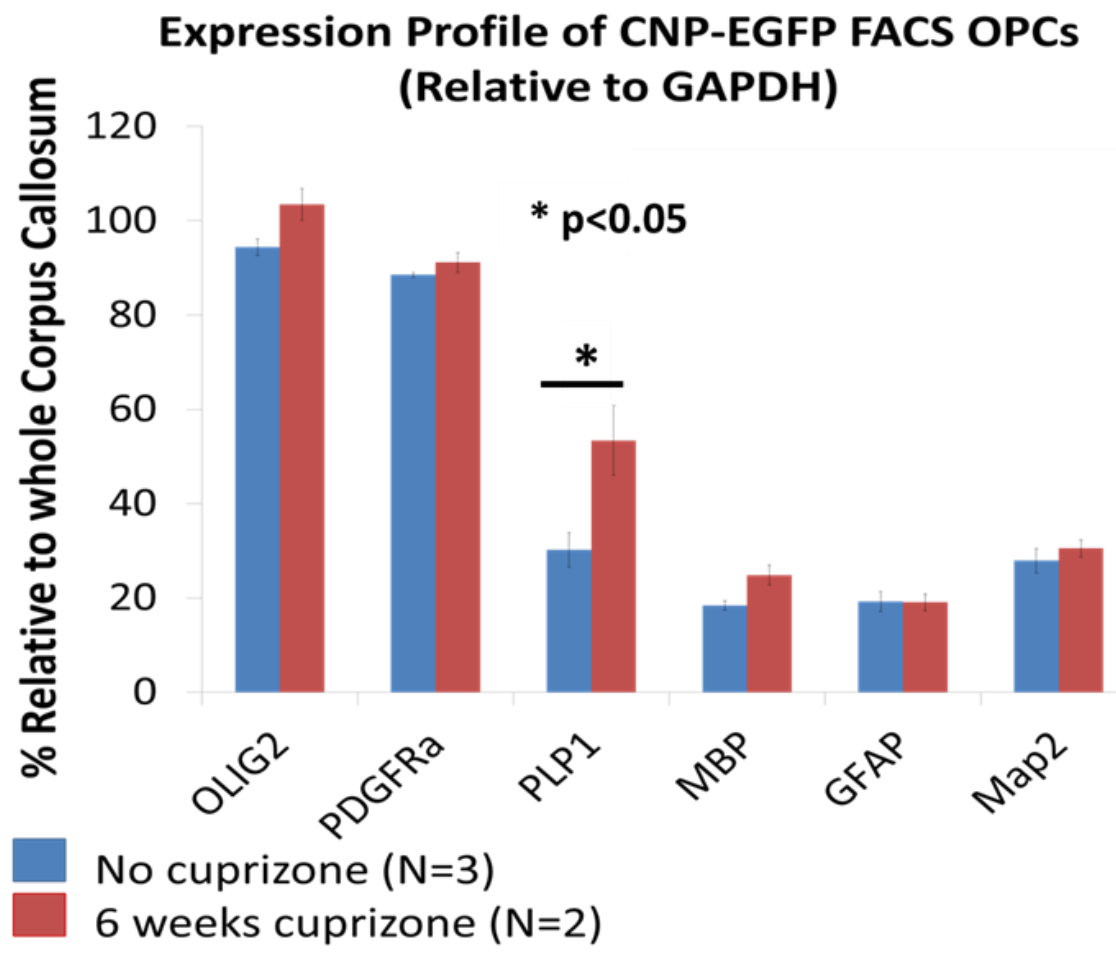


Figure 11. Transfection of $\mathrm{FAM}^{\mathrm{TM}}$ Dye-labeled pre- or anti-miR scrambled RNA controls into MEFs for FACS-based assessment of $\mathrm{FAM}^{+}$events. $\mathrm{FAM}^{+}$intensity from MEFs transfected with a pre- or anti-miRNA negative control was used to adjust threshold parameters (A). $\mathrm{FAM}^{+}$events relative to total events were calculated in real time with transfection efficiencies of $41.53 \% \pm 15.67 \%$ across experiments. Data are presented as mean \pm standard deviation. 
A Transfection Reagent Alone

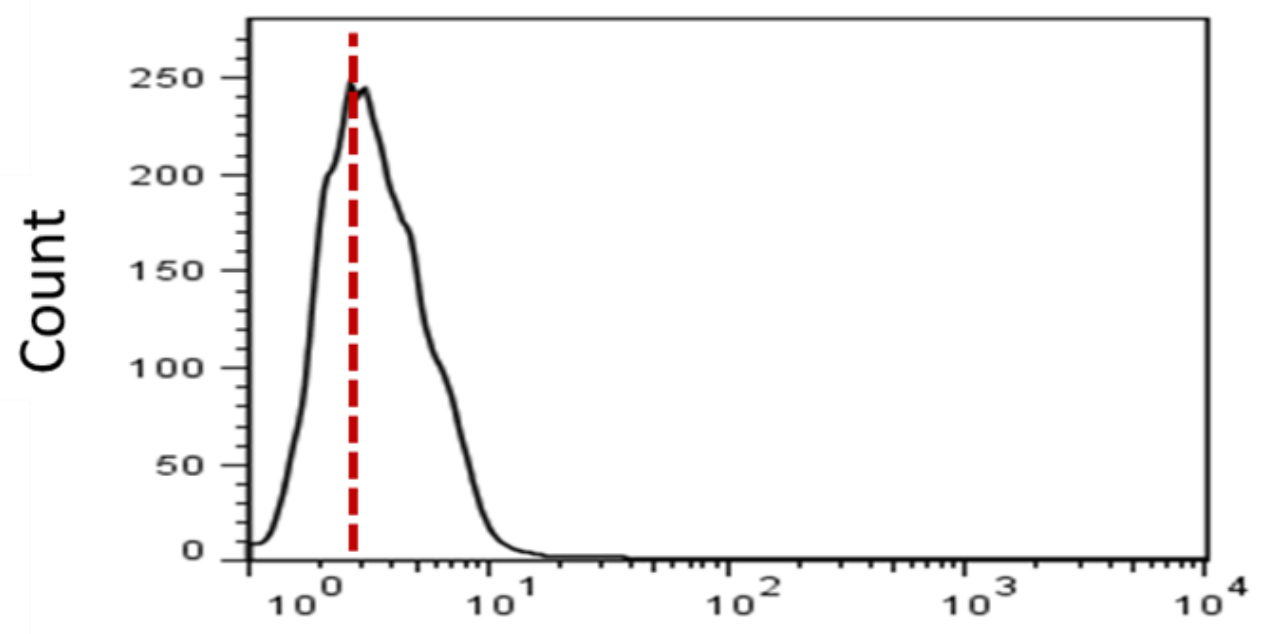

FAM (GFP) expression

B 0.1x FAM Labeled Reporter

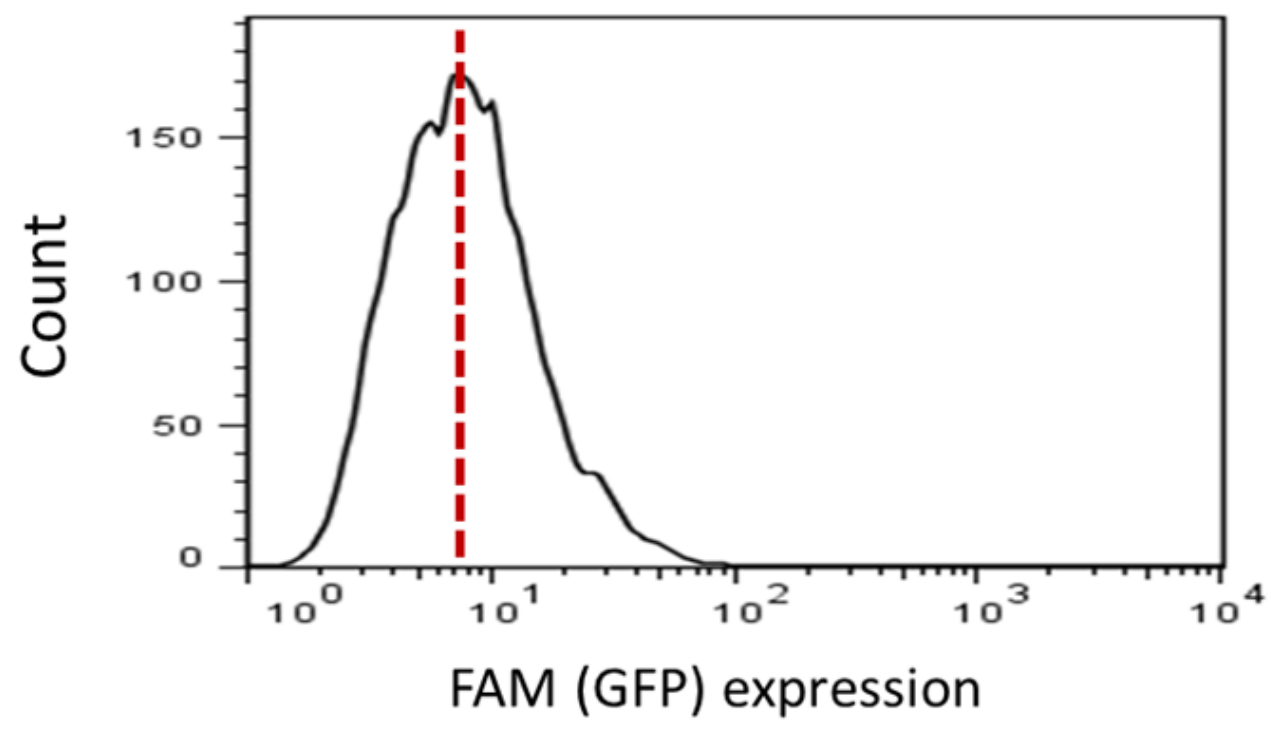


Figure 12. Sorted CNPase-EGFP ${ }^{+}$populations are not contaminated by myeloid lineage cells. Using callosal RNA isolated from mice following 6 weeks on cuprizone, no $\mathrm{CD} 45^{+}$cells were present when sorting with the perCP ${ }^{+}$conjugated antibody (gate R4; A). IgG-perCP isotype control expression was used to set threshold parameters (A). A negligible number of $\mathrm{CD}_{4} 5^{+} / \mathrm{CNPase}^{+}$cells were identified using the CD45-perCP antibody representing $<0.2 \%$ of sorted $\mathrm{CNPase}^{+}$events (gate R4; B). A subset of $\mathrm{CD}^{4} 5^{+} / \mathrm{CNPase}^{-}$cells were also identified suggesting the presence of systemically-derived myeloid lineage cells (B). 

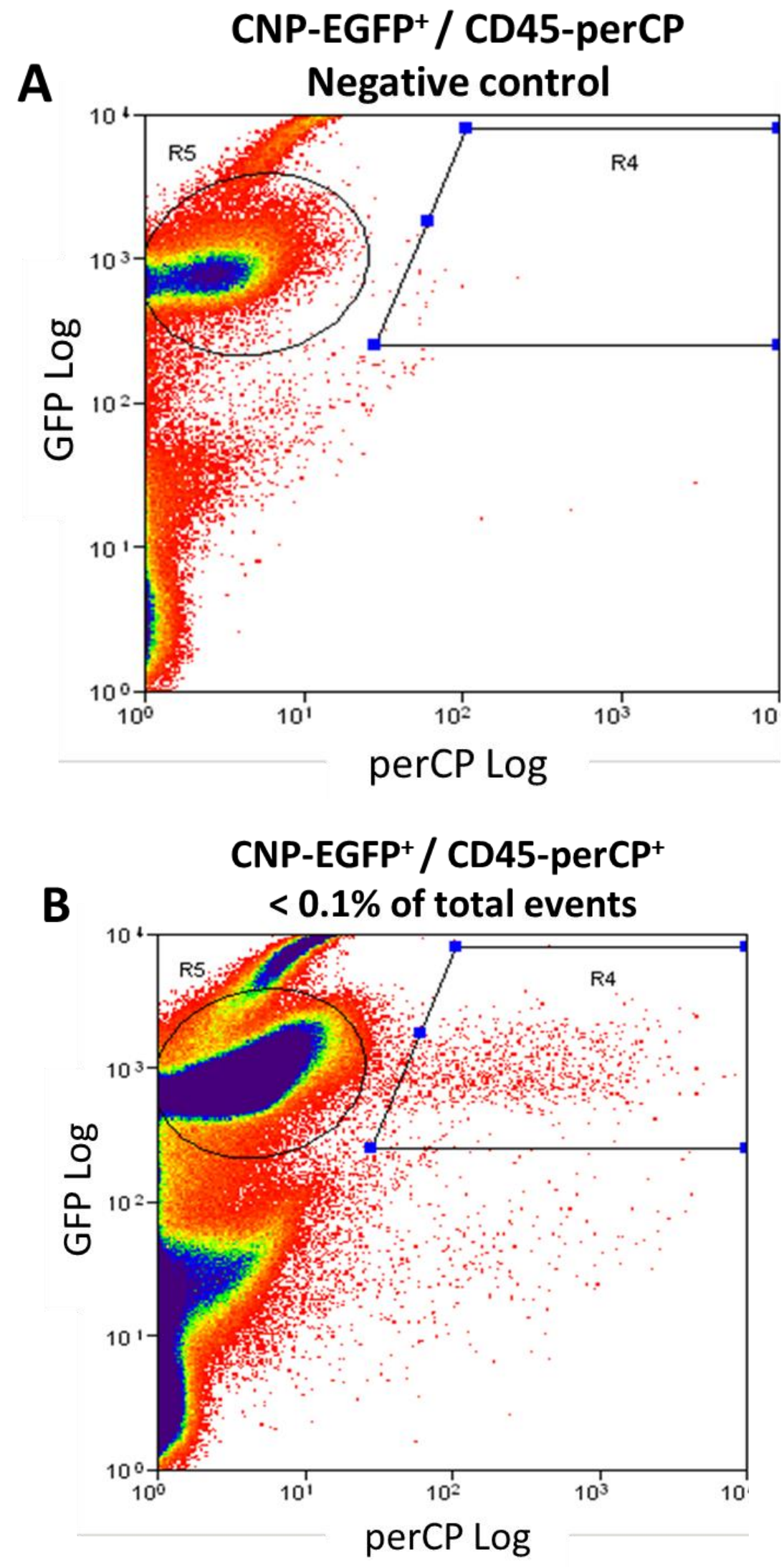
Figure 13. Table of miRNAs identified in the microarray also consistent with oligodendroglial-derived or white matter-derived miRNAs previously identified in the literature. List was comprised a priori and miRNAs only needed to be loosely associated with oligodendroglia. As miRNAs identified in while white with techniques that do not account for cell specificity, this list is representative of miRNAs with the potential to be oligodendroglial rather than oligodendroglialspecific. No other restrictive criteria were used. 
Present in Group ANOVA

\section{miRNA ID}

\begin{tabular}{|c|c|c|}
\hline & microarray & $p<0.05$ \\
\hline miR-7 & $x$ & $x$ \\
\hline $\operatorname{miR}-9$ & $x$ & \\
\hline miR-16 & $x$ & $x$ \\
\hline miR-17 & $x$ & $x$ \\
\hline miR-19b & $x$ & $x$ \\
\hline miR-20b & $x$ & $X$ \\
\hline miR-21 & $x$ & $x$ \\
\hline miR-23a & $x$ & $x$ \\
\hline miR-23b & $\mathrm{X}$ & $\mathrm{X}$ \\
\hline miR-29a & $x$ & \\
\hline miR-34a & $\mathrm{X}$ & \\
\hline miR-103 & $x$ & \\
\hline miR-106a & $x$ & $X$ \\
\hline miR-107 & $x$ & \\
\hline miR-124 & $x$ & $X$ \\
\hline miR-130a & $x$ & $x$ \\
\hline miR-138 & $x$ & $x$ \\
\hline miR-140 & $x$ & \\
\hline miR-145 & $x$ & $X$ \\
\hline miR-155 & $x$ & $x$ \\
\hline \multicolumn{3}{|l|}{ miR-192 } \\
\hline \multicolumn{3}{|l|}{ miR-199a } \\
\hline miR-205 & $\mathrm{X}$ & $\mathrm{X}$ \\
\hline miR-219 & $x$ & $x$ \\
\hline miR-326 & $x$ & $x$ \\
\hline miR-338 & $x$ & $x$ \\
\hline \multicolumn{3}{|l|}{ miR-381 } \\
\hline miR-650 & & \\
\hline
\end{tabular}


Figure 14. Microarray data reveals that Sfmbt2 cluster miRNAs are significantly decreased at 6 weeks on cuprizone compared to no cuprizone miRNA expression levels. A Z-score heat map displaying the number of standard deviations a particular miRNA's expression is from the mean expression of all mIRNAs identified above background in the microarray (A). OP miRNA expression profiles are dynamically regulated during cuprizone-induced demyelination ( 3 and 6 weeks on cuprizone; $A$ ) and remyelination ( 3 and 6 weeks off cuprizone; A). Pooled samples displayed consistent miRNA expression values across samples of a particular cuprizone treatment condition (A). Over half of the top 25 most significant miRNAs were members of the Sfmbt2 miRNA cluster, a recently identified group of miRNAs located within intron 10 or 11 of the Sfmbt2 host gene (A). Fold change relative to the no cuprizone group was assessed for 3 and 6 weeks on and 3 and 6 weeks off cuprizone revealing that nearly all Sfmbt2 cluster miRNAs share a similar expression profile across cuprizone treatment conditions (B). 


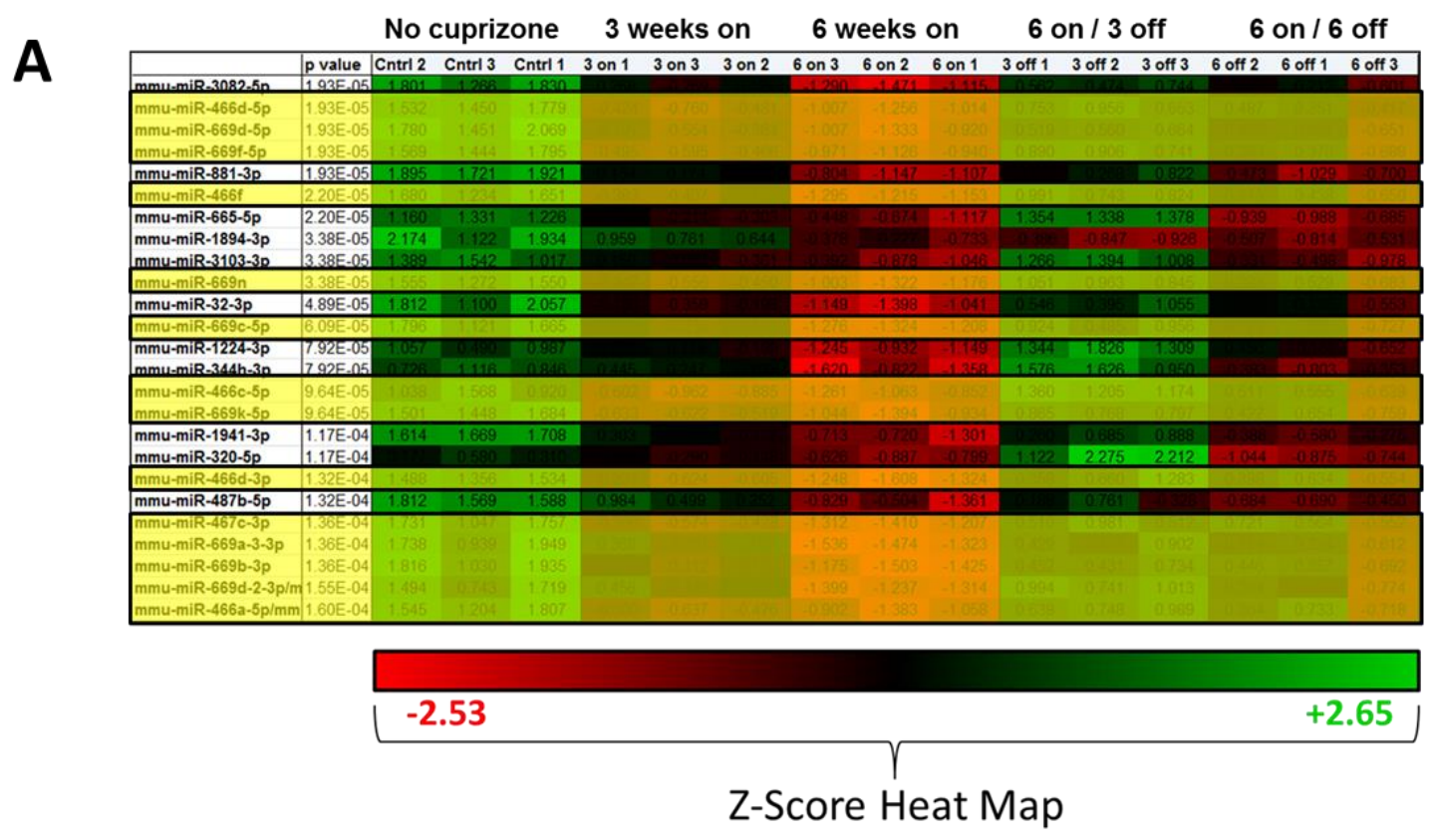

B

3 on/ 3 on/

\section{miRNA ID}

3 on/ 3 on/

3 on 6 on 3 off 6 off

mmu-miR-297a-3p/mmu-miR-297b-3p/

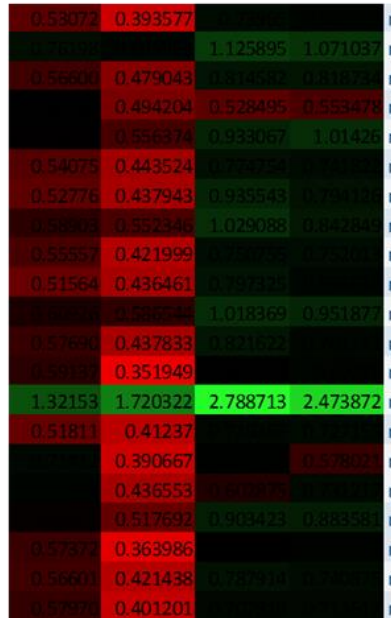

mmu-miR-297c-5p

mmu-miR-466a-3p/mmu-miR-466b-3p/

mmu-miR-466a-3p/mmu-miR-466e-3p

mmu-miR-466a-5p

mmu-miR-466a-5p/mmu-miR-466p-5p

mmu-miR-466b-5p/mmu-miR-466o-5p

mmu-miR-466c-5p

mmu-miR-466d-3p

mmu-miR-466d-5p

mmu-miR-466e-5p

mmu-miR-466f

$m m u-m i R-466 f-3 p$

mmu-miR-466h-5p

mmu-miR-466i-3p

mmu-miR-466i-5p

mmu-miR-466m-3p

mmu-miR-466m-5p/mmu-miR-669m-5

mmu-miR-466q

mmu-miR-467a-3p

mmu-miR-467b-3p

\section{3 on 6 on 3 off 6 off}

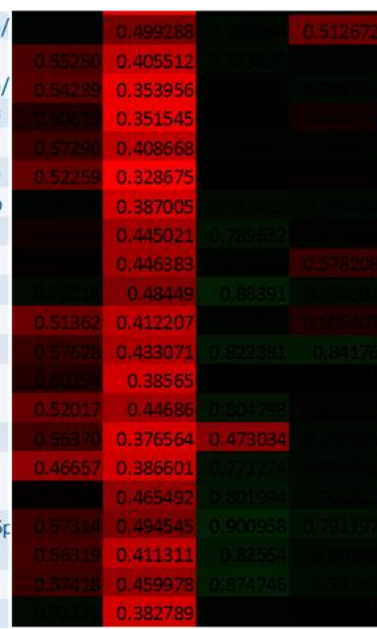

mmu-miR-467e-5p

mmu-miR-467f

mmu-miR-467g

mmu-miR-669a-3-3p

mmu-miR-669a-3p/mmu-miR-669o-3

mmu-miR-669b-3p

mmu-miR-669c-3p

mmu-miR-669c-5p

mmu-miR-669d-2-3p

mmu-miR-669d-2-3p/mmu-miR-669d

mmu-miR-669d-5p

mmu-miR-669e-3p

mmu-miR-669f-3p

mmu-miR-669f-5p

mmu-miR-669i

mmu-miR-669k-5p

mmu-miR-669l-3p

mmu-miR-669l-5p

mmu-miR-669m-3p

mmu-miR-669n

mmu-miR-669p-3p 
Figure 15. Sfmbt2 cluster member miR-297c displays a significant increase concomitant with differentiation in primary OPs. 24 hours post-MACS isolation, $93.94 \% \pm 6.84 \%$ of total Hoechst ${ }^{+}$nuclei were also A2B5+ (C) and $83.24 \% \pm$ $9.35 \%$ were $\mathrm{O}^{+}(\mathrm{A}$ and $\mathrm{C})$. Following 72 hours of differentiation with mitogen removal and T3 addition to the culture medium, process elongation and arborization were accompanied by a reduced percentage of $\mathrm{A}_{2} \mathrm{~B}^{+}$to $14.87 \% \pm$ $7.77 \%(\mathrm{C})$ and $\mathrm{O}^{+}$cells $46.23 \% \pm 7.77 \%(\mathrm{C})$ relative to total $\mathrm{Hoechst}^{+}$nuclei.

$\mathrm{O} 1^{+}$cells were not present 24 hours post-MACS (C) but were identified following 72 hours differentiation constituting $41.88 \% \pm 18.64 \%$ of total Hoechst $^{+}$nuclei (B and C). Antibodies against GFAP, an intermediate astrocyte filament, demonstrated GFAP $^{+}$immunoreactivity 24 hours post-MACS or following 72 hours proliferation to be $1.54 \% \pm 0.05 \%$ of total Hoechst ${ }^{+}$nuclei (A and C). Following 72 hours of differentiation, GFAP expression increased to $27.50 \% \pm$ $8.83 \%$ of total Hoechst ${ }^{+}$nuclei (B and C). As a positive miRNA control to differentiation, miR-338-5' was assessed and demonstrated to be significantly increased in primary mouse OPs following 72 hours of differentiation compared to 72 hours of proliferation (B and D). Of all Sfmbt2 cluster miRNAs assayed, only miR-297c was significant, consistently displaying expression levels of 3 fold or higher in differentiating mouse OPs relative to proliferating mouse OPs (D). Data in $D$ are presented as mean \pm standard deviation; ${ }^{*} p<0.05$. Scale bars in $A$ and $B=100 \mu \mathrm{m}$. 

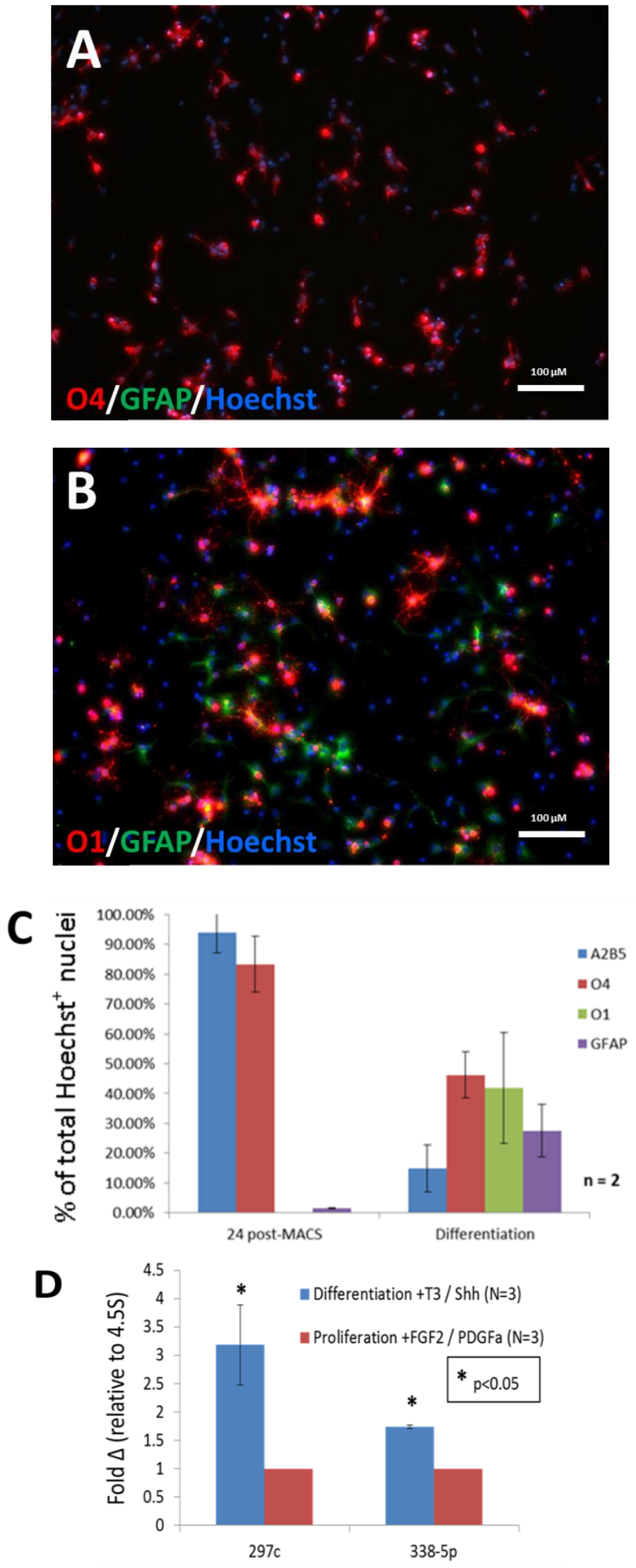
Figure 16. miR-297c transfection regulates cell cycle progression in MEFs. Multiple target prediction algorithms predicted miR-297c to target the cyclin T2, a regulator of $\mathrm{G}_{1}$ to $\mathrm{S}$-phase transition. Provided is a mechanistic schematic of potential regulatory control of cell cycle by miR-297c induction. Normally in mitotic cells, cyclin T and CDK9 form a complex (P-TEFb) which can then interact with BRD4 which promotes P-TEFb recruitment to $G_{1}$ genes thus favoring $G_{1}$ to $S$-phase transition (A). Following induction of miR-297c, cyclin $T$ transcripts are targeted by miR-297c thus repressing cyclin $T$ translation (B). 
A

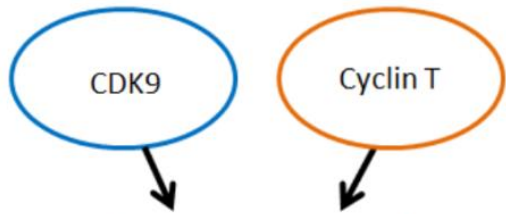

P-TEFb complex formation
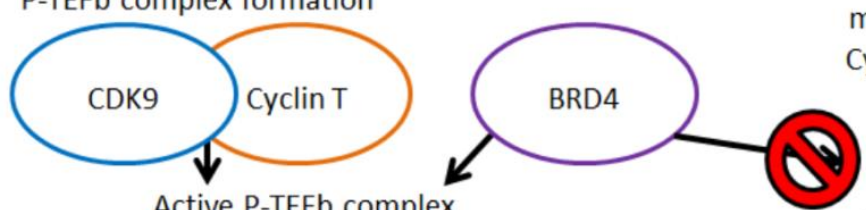

miR-297c prevents

Cyclin T translation

Cyclin T
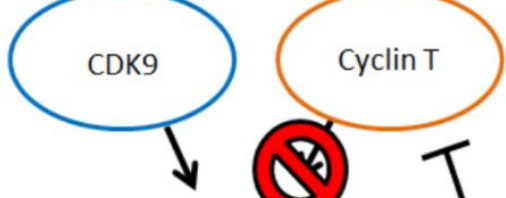

Active P-TEFb complex
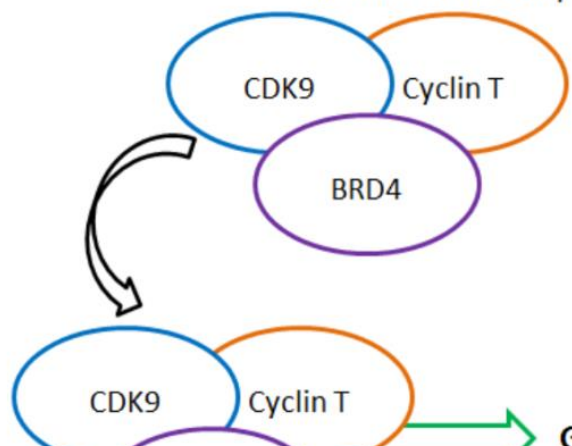

$\mathrm{G}_{1}$ to $\mathrm{S}$-phase transition

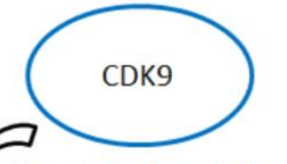

miR-297c

Thus, P-TEFb complex

formation is inhibited

preventing BRD4

mediated activation

Compromised P-TEFb complex

formation results in failed

recruitment to $G_{1}$ genes and

thus $G_{1}$ cell cycle arrest

BRD4

$\mathrm{G}_{1}$ gene DNA

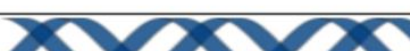


Figure 17. miR-297c regulates S-phase transition in cycling MEFs. Cell cycle analysis revealed that 48 hours following miR-297c or control transfection, MEFs transfected with miR-297c displayed a significant increase in $G_{1} / G_{0}$ frequencies (A) and a significant reduction in S-phase frequencies (B). Data in $A$ and $B$ are presented as mean frequency \pm standard deviation; ${ }^{*} p<0.05$. 


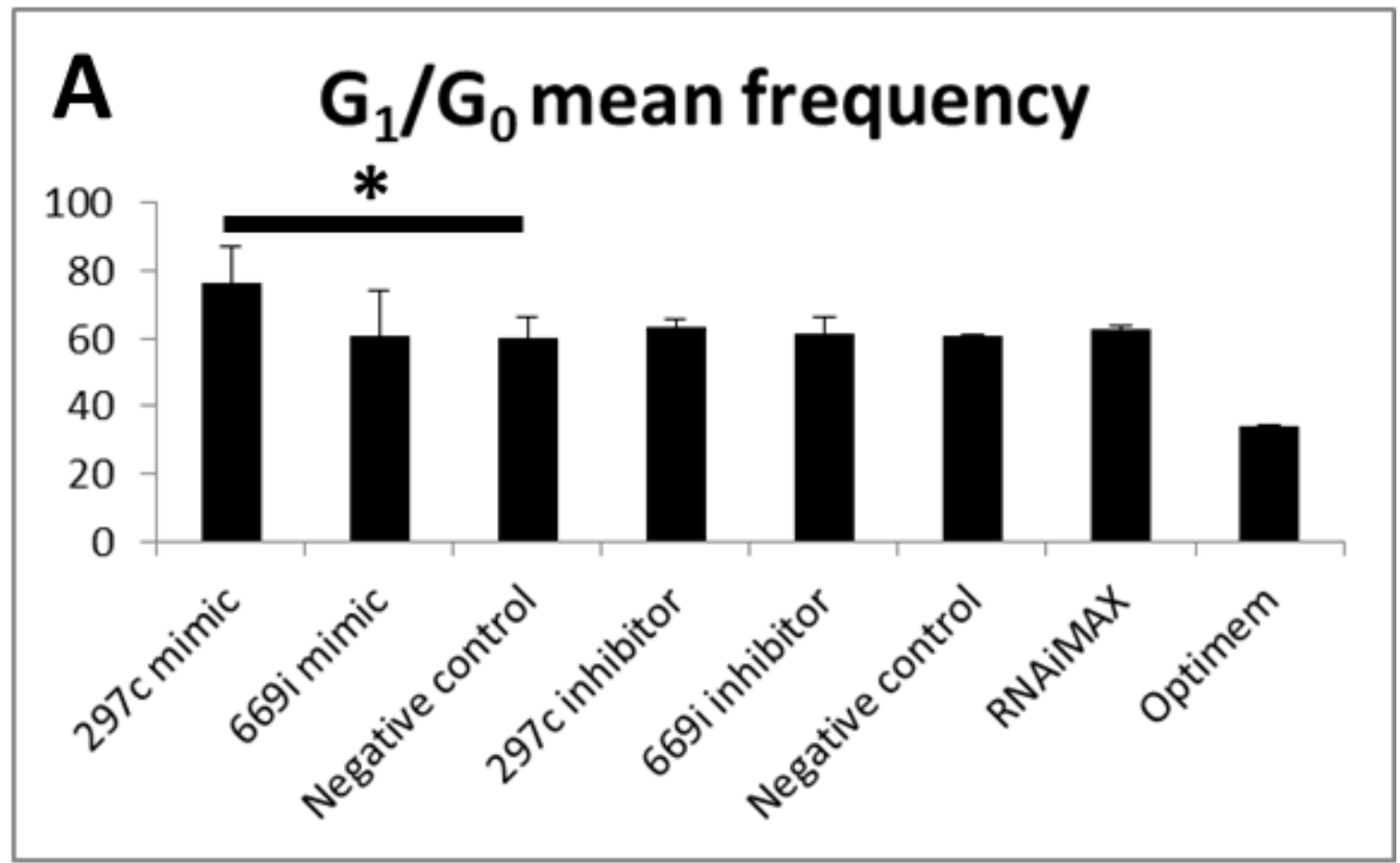

B S-phase mean frequency

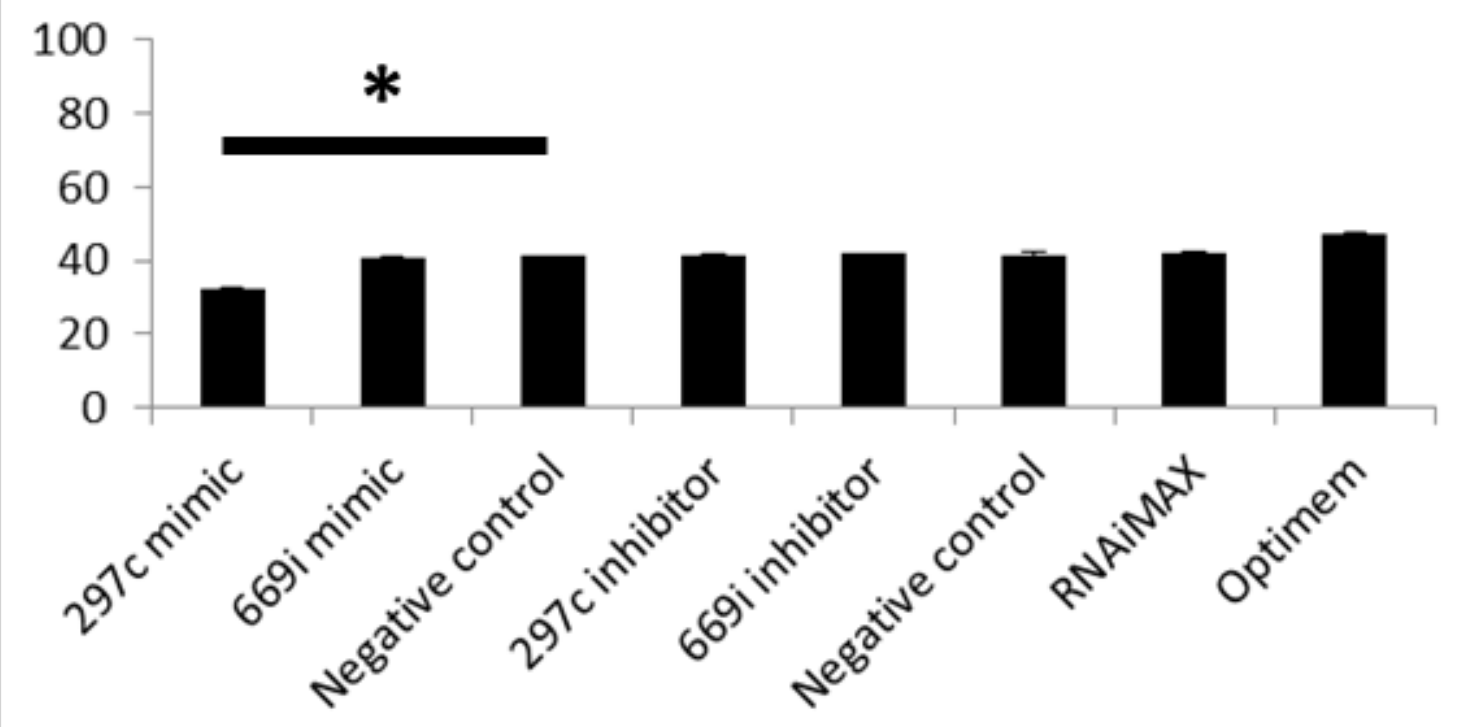




\section{CHAPTER 5}

\section{REGENERATIVE POTENTIAL OF THE CNS AND ITS CLINICAL LIMITATIONS}

\section{CNS Regeneration}

The dogmatic idea that CNS damage results in permanent cell loss and chronic dysfunction or death has existed for thousands of years, even dating back to Egyptian times (Eltorai, 2003). Only in the last several decades have we begun to understand the regenerative potential of the CNS, which is not equal in all cell types and often remains incomplete (Silver, 2009). There are several neurogenic niches in the adult mammalian CNS which harbor mitotically competent cells able to respond to cell loss by replicating themselves, migrating to the site where they are needed, differentiating into one of many CNS cell types and finally integrating back into the CNS (Horner and Gage, 2000). As the focus of this manuscript is oligodendroglial lineage cells, further discussion concerning regeneration and functional recovery will be from the perspectives of remyelination and demyelination, respectively. The regenerative potential of white matter is actually rather incredible. CNS myelinogenesis continues well after birth with ongoing oligodendroglial development demonstrating the highly dynamic and plastic glial environment (Dubois et al., 2013). Additionally, there is simultaneous turnover of 
the already mature OLs which are constantly being replaced by resident OPs. This process proceeds without limitation during normal myelin maintenance (Young et al., 2013). However, following trauma- or disease-induced demyelination, the number of axons which lack myelin is too great and OPs are not available in the quantities required for complete remyelination. As a result, the dysfunction associating with demyelination is never fully restored and persists chronically. This highlights the importance of research attempting to enhance remyelination when the endogenous regenerative response is sub-optimal.

\section{The Modalities of Demyelination}

The data presented in Chapters $2-4$ demonstrate that demyelination is accompanied by complex and widespread changes at both the whole organism level (Chapters 2 and 3) and at the molecular level (Chapter 4). These diseaseoriented changes manifest as multiple modalities. Behavioral dysfunction, tissue loss (axonal and glial), cellular changes and compromised molecular events are all major components of the post-demyelination sequelae but ultimately they cannot be individualized as there is a connectivity present between all of these modalities which ultimately manifests as disease pathology. Although the complexity and interworking of that interconnectivity are not completely understood, it is safe to assume that until all are ameliorated, remyelination will not be complete. Most therapeutic remyelination strategies target only one of these modalities. This is not out of ignorance as experimentally evaluating more 
than one of the above mentioned issues begins to introduce too many variables, making experimental results difficult to interpret thus compromising parsimony. When only a single variable is manipulated, and assuming experimental design is acceptable, parsimony will be maintained. So the question stands, "Is it nonparsimonious to simultaneously approach an experimental design from modalities ranging from behavior for molecular biology?" It may be. However, it must also be considered that the most effective experimental design to wholly address a complex, multi-factorial disease such as demyelination, may not be the most parsimonious.

Studies maintaining maximal parsimony which perform few manipulations and focus on two or less modalities, are crucial to understanding particular fundamental aspects of oligodendroglial biology and the biology of all cell types involved in remyelination for that matter. Even the experiments detailed here in Chapters $2-4$ were completed using limited modality experimental designs. But rather than attempting to translate a single treatment strategy from bench to bedside entirely within one modality, it may be more advantageous to combine multiple treatment strategies from across different modalities (i.e. manipulating the molecular landscape of cells, manipulating the identity of populations of cells, manipulating the host environment prior to and following transplantation to promote survival, transplanting cells to replace lost tissue, managing immune rejection concerns and providing training /rehab and physical therapy to facilitate these newly transplanted cell's integration into the CNS). Therefore, there is a 
need for studies which combine multiple treatment strategies all previously demonstrated to be effective on their own into the same model of demyelination. The hope would be that an additive or synergistic effect for remyelination would be observed thereby maximizing recovery.

\section{Treating Demyelination Clinically}

Treating demyelination so that lost function is completely restored is an arduous task. In fact to date, despite the wealth and breadth of data demonstrating successful remyelination in experimental models, no treatment currently exists where the patient's cognitive ability, motor and sensory function, financial burden and overall quality of life were all restored to pre-injury levels following treatment. This does not take away from the incredible advancements which have been made both experimentally and clinically, but rather points out that despite all of the progress, we are still very far from a complete "cure". Moreover, this phenomenon is not exclusive to the demyelinating disorders as no single "cure" is available clinically for any neurological condition, including but not limited to: SCI and TBI (from non-myelinogenic perspectives), Parkinson's, Alzheimer's, Huntington's, amyl lateral sclerosis (ALS) as well as all of the genetic and acquired demyelinating disorders. Concerning neurological disease in general, progress has not been absolutely absent however as treatment options which slow disease progression and manage symptomology are available for other non-myelinogenic disorders of the CNS. For example, remarkable progress has 
been made in the field of $\mathrm{SCl}$ (a traumatic event involving demyelination) where epidural stimulation of the spinal cord below the level of the lesion resulted in substantial functional recovery in patients with otherwise very poor prognoses (Harkema et al., 2011). However, the nature of this recovery is independent of remyelination as there is no documented evidence suggesting a link between rehabilitation / physical therapy and remyelination. Thus, treatment options for the myelinogenic disorders remain very limited with the development of a true "cure" being even more elusive.

The mere idea of a true "cure" for any neurological disease may be naïve as there may be no single therapeutic strategy which will completely "cure" an otherwise chronic CNS insult. As mentioned above, demyelination is accompanied by complex and widespread changes at both the whole organism and the molecular level. This brings up two important considerations: 1 . such widespread changes may be too dramatic and disruptive for complete remyelination to occur making any attempts at recovery a futile pursuit; 2 . in cases where there is at least some capacity for remyelination, it also needs to be considered that there is a limit to the extent of remyelination possible. The extent of remyelination possible would be determined by the cause of and extent of demyelination. In both cases, unless progress is attempted under the assumption that complete remyelination is achievable, understanding when a pursuit is futile and becoming aware of the existence of and limitations provided by such a ceiling will remain unknown. 
There are multiple treatment options which are hopeful for the treatment of demyelination but they can essentially be arranged into two categories: 1 . using pharmacological-based methods to enhance the endogenous remyelination response; 2. transplanting OPs into the demyelinated white matter in order to augment remyelination. Targeting endogenous OPs pharmacologically in an effort to facilitate remyelination by favoring oligodendroglial versus astroglial differentiation has some promise as a treatment for demyelination as it would accomplish the following: Limit astrogliosis (an inhibitor of remyelination) and provide more myelinating OLs. However, although shifting the astrocyte:oligodendrocyte ratio of transplanted cells to favor oligodendroglial differentiation would most likely provide some benefit to remyelination, the primary issue of too few OPs present still remains.

Targeting endogenous OPs with treatments which would enhance their proliferation and the overall numbers available for remyelination could possibly improve the number of cells available in progenitor pools for remyelination. However, manipulating proliferation may also be accompanied by potentially fatal contraindications. For example, increasing proliferation in an already mitotically competent cell population may favor the in vivo selection of cells with a greater proliferative capacity. This capacity for proliferation may also be biologically synonymous with a capacity for tumorigenicity. When considering that glioblastomas (the most invasive form of brain cancer) are the result of aberrantly 
cycling OPs, it may be unwise to pursue any treatment option clinically where enhanced proliferation is the ultimate goal as it would be unsafe and irresponsible to patients. Another possible side effect would be that the majority of the newly formed OPs would favor an astrocytic fate and ultimately contribute to glial scaring providing a barrier to remyelination. In both cases, pharmacologically targeting OPs to enhance proliferation fails to result in improved remyelination and may actually result in a poorer outcome that no treatment at all.

Of all the options to improve remyelination mentioned above, the most realistic is cell transplantation. As detailed in Chapter 1, there are multiple potential sources for engrafted cells which can be transplanted into demyelinating lesions. The limited availability of both fetal and adult tissue has eliminated the possibility of using these tissues as a reliable source of OPs for clinical purposes. As a result the field has shifted to deriving OPs from human ES (hES) and induced pluripotent stem (iPS) cell sources. Both obviate the need for an OP source that is limitlessly scalable for any experimental and/or clinical purposes. However, iPS-derived OPs in culture exhibit much less teratoma formation than hESderived OPs and can be generated from the graft recipient. Additionally, human iPS cell-derived OPs reach senescence more rapidly that hES cell-derived OPs which reduced the time and resources involved with scalable expansion protocols. These two advantages make iPS cell-derived OPs the superior option for transplantation compared to ES or tissue-derived OPs. Moreover, prior to this 
being adopted in clinics nationwide as a means to treat demyelination, much more stringent differentiation protocols must be established and flawless purification techniques must be attainable for both hES and iPS cell-based approaches. When considering that it may only take a single abhorrently proliferating transplanted cell to result in tumor replication and that the host immune system will already be compromised due to graft-promoting immunosuppression, these technical considerations are of the utmost importance. Until that time, OP transplantation in the clinic may remain absent.

Even if transplantation strategies are used, this does not address all of the different dysfunctional modalities involved in demyelination. Remyelination by way of transplantation may also require a combinatorial approach where cell replacement strategies which replace lost tissue must be combined with at least rehabilitation and physical therapy to promote integration into the CNS. Cell transplantation strategies must maximize the volume of tissue restored while rehabilitation strategies and physical therapy must facilitate integration of the newly transplanted cells into the dysfunctional CNS making it indistinguishable from normal CNS tissue. Therefore, aside from attempting to optimize both transplantation- and rehabilitation-based treatment options, a more concerted effort needs to be made to combine treatments across multiple modalities in a single patient to assess the potential synergistic effects of such approaches. More importantly these strategies may maximize the patient's chance at an improved quality of life. 


\section{miRNAs as Therapeutic Targets: Prospective Treatment Strategies for Demyelination}

The small size of miRNAs allows a single miRNA to target multiple mRNAs (Hu et al., 2012). The implications of this unique biological characteristic are that widespread effects on translation and ultimately cellular function can be achieved by a drug which introduces or inhibits expression of one to several miRNAs. This makes them attractive therapeutic targets as manipulating a single miRNA could regulate translation of multiple signaling molecules in a pathway as well as multiple signaling molecules in multiple pathways. In the case of demyelinating disease, as demonstrated in Chapter 4, oligodendroglial miRNA dysregulation accompanies demyelination. Identifying those miRNAs which are dysregulated may be advantageous to the development of treatments which aim to restore aberrant miRNAs to normal levels thus providing therapeutic benefit.

A variety of in vivo methods have been described for the delivery of miRNAs or molecules to inhibit them (Hu et al., 2012). Intranasal administration of an antimiR-126 molecule conjugated to cholesterol successfully treated respiratory disease in mice by negatively regulating T-helper-2 cytokine production (Mattes et al., 2009). Others have delivered antisense oligonucleotide against miR-122 (anti-miR-122) systemically in mice finding that it reduced miR-122 levels resulting in a therapeutic benefit in obese mice (Esau et al., 2006). Furthermore, 
in vivo administration of lentiviral miR-26a inhibited nasopharyngeal

tumorigenicity in nude mice (Lu et al., 2011). Moreover, systemically delivering miR-16 slowed metastatic prostate tumor growth by down-regulating multiple target mRNAs involved in cell cycle control thus preventing the translation of multiple cell cycle proteins translation in a xenograft model (Takeshita et al., 2010). Although experimental progress has been promising in animal models, clinical trials are necessary to understand what potential there is for miRNA manipulation in human demyelination (Kasinski and Slack, 2011).

Exosomes have also been suggested to be an efficient means of miRNA delivery. Due to their ability to efficiently shuttle small molecules between cells, exosomes are an extremely promising therapeutic tool for numerous diseases. Exosomes have been demonstrated to target specific cell types. Exosomes loaded with synthetic miRNA (siRNA) targeting Gapdh were injected into mice, Gapdh was specifically knocked down in the appropriate cell types, including neurons, microglia and OLs (Alvarez-Erviti et al., 2011). With the technological advances made in the field of exosomal biology, we are currently able to manipulate the contents within the exosome to deliver miRNAs favorable to remyelination. Moreover, it is also possible to manipulate the exosomal membrane proteins thus allowing for the cell-specific targeting or miRNA contents to areas of demyelination. For instance, exosomes engineered so that the PDGFR $\alpha$ binding domain of PDGFa is membrane bound and extracellularly exposed, exosomes with a miRNA payload favorable to remyelination could be 
delivered to PDGFR $\alpha$ expressing OPs intrathecally, within a demyelinating lesion or even systemically. Of all delivery methods available for miRNA manipulations, exosomal-based miRNA delivery methods have the most promise in clinical trials because of the potential for tissue specificity and safety of exosomal use in humans (Hu et al., 2012).

As mentioned in Chapter 1 and here in Chapter 5, when attempting to completely restore lost myelin to pre-demyelination levels, any strategy to enhance remyelination should begin with the transplantation of OPs into the demyelinated lesion. However, data presented in Chapter 4 demonstrates that demyelination is also accompanied by global miRNA dysregulation. Therefore, the manipulation of miRNAs to enhance remyelination should be applied in conjunction with OP transplantation. The most logical platform to achieve this would be an ex vivo approach where miRNAs or miRNA inhibitors are introduced into the OPs prior to engraftment. One group recently demonstrated that following tail veil injection of macrophages that have been manipulated ex vivo to express miR-143, miR-143 was detectable in blood and cerebrospinal fluid (CSF; Akao et al., 2011). These data suggest that ex vivo approaches might carry much promise for the introduction of miRNAs favorable to remyelination in OPs prior to transplantation into a demyelinated lesion.

\section{miRNAs as Biomarkers of Demyelination}


There are currently 178 clinical trials listed involving miRNAs (clinicaltrials.gov). However, none of them are evaluating miRNA manipulation as a therapy. Rather, studies are focused on either gathering tissue to identify intracellular miRNA dysregulation from patient derived sources or attempting to detect miRNA levels in blood, cerebrospinal fluid (CSF) and from tissue biopsies for use as a diagnostic tool (Ciesla et al., 2011). With respect to the diagnostic potential of miRNAs, aberrant miRNAs have been identified in multiple diseases (Taylor and Gercel-Taylor, 2008; Rabinowits et al., 2009). The source of these miRNAs is exosomes released endogenously from diseased cells. Exosomes from diseased cell have miRNA expression profiles which are distinct from normal cell exosomal miRNA expression profiles. Eventually, these exosomes ultimately make their way to blood or CSF and are released allowing for their detection. Although several clinical trials have shown that miRNAs can be diagnostic tools for neurological disease, no diagnostic miRNAs have been identified in patients with a demyelinating disease.

Additionally, rather than look at miRNA manipulation directly as a treatment option, we can use them to develop targets to demyelinating disease. In addition to data presented in Chapter 4, oligodendroglial miRNA dysregulation has been described in the molecular sequelae underlying demyelination in adult-onset autosomal dominant leukodystrophy (ADLD; Lin and Fu, 2009). It is likely that oligodendroglial miRNA dysregulation plays a role in most if not all demyelinating diseases. Therefore, determining differences in miRNA expression in patients 
with a demyelinating disorder compared to the normal population, may reveal dysregulated miRNAs that play a crucial role in demyelination. The mRNA targets of those miRNA and their biological function in disease pathology can be determined experimentally thereafter allowing for the development of treatment strategies which may or may not involve miRNAs.

\section{Summary and Final Thoughts}

Here we present a comprehensive demonstration of the behavioral, systemslevel, cellular and molecular dysfunction associated with demyelination. Although current treatment options do not show substantive efficacy, there is much promise on the horizon for remyelination therapeutics as biological and technological advances have allowed for the development of OP transplantationbased strategies and miRNA-based strategies, respectively. Whichever therapeutic strategy(s) is employed, it is imperative that it is done early. Certainly there is most likely a window that could be considered too early and intervening before this point would be detrimental. However, as described in Chapter 1, OLs have an important role in axonal health. Following demyelination, as an axon remains demyelinated, the lack of oligodendroglial support will continue. It is inevitable that a chronic removal of glial-derived axonal support will ultimately reduce axon viability if not resulting in axon loss. Moreover, axon loss has been documented following chronic demyelination (Lovas et al., 2000). Therefore, the 
earliest intervention that can be responsibly and safely made must be attempted as remyelination is only as possible as the number of axons which remain viable. 


\section{REFERENCES}

Adlard PA, Perreau VM, Cotman CW (2005) The exercise-induced expression of BDNF within the hippocampus varies across life-span. Neurobiology of aging 26:511-520.

Akao Y, lio A, Itoh T, Noguchi S, Itoh Y, Ohtsuki Y, Naoe T (2011) Microvesiclemediated RNA molecule delivery system using monocytes/macrophages.

Molecular therapy : the journal of the American Society of Gene Therapy 19:395399.

Alexander WS (1949) Progressive fibrinoid degeneration of fibrillary astrocytes associated with mental retardation in a hydrocephalic infant. Brain : a journal of neurology 72:373-381, $373 \mathrm{pl}$.

Alluin O, Karimi-Abdolrezaee S, Delivet-Mongrain H, Leblond H, Fehlings MG, Rossignol S (2011) Kinematic study of locomotor recovery after spinal cord clip compression injury in rats. Journal of neurotrauma 28:1963-1981. 
Almeida S, Zhang Z, Coppola G, Mao W, Futai K, Karydas A, Geschwind MD, Tartaglia MC, Gao F, Gianni D, Sena-Esteves M, Geschwind DH, Miller BL, Farese RV, Jr., Gao FB (2012) Induced pluripotent stem cell models of progranulin-deficient frontotemporal dementia uncover specific reversible neuronal defects. Cell reports 2:789-798.

Alvarez-Erviti L, Seow Y, Yin H, Betts C, Lakhal S, Wood MJ (2011) Delivery of siRNA to the mouse brain by systemic injection of targeted exosomes. Nature biotechnology 29:341-345.

Ambros V (2004) The functions of animal microRNAs. Nature 431:350-355. Armstrong R, Friedrich VL, Jr., Holmes KV, Dubois-Dalcq M (1990) In vitro analysis of the oligodendrocyte lineage in mice during demyelination and remyelination. The Journal of cell biology 111:1183-1195.

Assadi M, Janson C, Wang DJ, Goldfarb O, Suri N, Bilaniuk L, Leone P (2010) Lithium citrate reduces excessive intra-cerebral $\mathrm{N}$-acetyl aspartate in Canavan disease. European journal of paediatric neurology : EJPN : official journal of the European Paediatric Neurology Society 14:354-359.

Azim K, Raineteau O, Butt AM (2012) Intraventricular injection of FGF-2 promotes generation of oligodendrocyte-lineage cells in the postnatal and adult forebrain. Glia 60:1977-1990. 
Back SA, Tuohy TM, Chen H, Wallingford N, Craig A, Struve J, Luo NL, Banine F, Liu Y, Chang A, Trapp BD, Bebo BF, Jr., Rao MS, Sherman LS (2005) Hyaluronan accumulates in demyelinated lesions and inhibits oligodendrocyte progenitor maturation. Nature medicine 11:966-972.

Baek D, Villen J, Shin C, Camargo FD, Gygi SP, Bartel DP (2008) The impact of microRNAs on protein output. Nature 455:64-71.

Bakhti M, Snaidero N, Schneider D, Aggarwal S, Mobius W, Janshoff A, Eckhardt M, Nave KA, Simons M (2013) Loss of electrostatic cell-surface repulsion mediates myelin membrane adhesion and compaction in the central nervous system. Proceedings of the National Academy of Sciences of the United States of America 110:3143-3148.

Bambakidis NC, Miller RH (2004) Transplantation of oligodendrocyte precursors and sonic hedgehog results in improved function and white matter sparing in the spinal cords of adult rats after contusion. The spine journal : official journal of the North American Spine Society 4:16-26.

Barca-Mayo O, Lu QR (2012) Fine-Tuning Oligodendrocyte Development by microRNAs. Frontiers in neuroscience 6:13. 
Bartel DP (2009) MicroRNAs: target recognition and regulatory functions. Cell 136:215-233.

Bartel DP, Chen CZ (2004) Micromanagers of gene expression: the potentially widespread influence of metazoan microRNAs. Nature reviews Genetics 5:396400.

Bartzokis G, Lu PH, Tingus K, Mendez MF, Richard A, Peters DG, Oluwadara B, Barrall KA, Finn JP, Villablanca P, Thompson PM, Mintz J (2010) Lifespan trajectory of myelin integrity and maximum motor speed. Neurobiology of aging 31:1554-1562.

Basso DM, Beattie MS, Bresnahan JC (1995) A sensitive and reliable locomotor rating scale for open field testing in rats. Journal of neurotrauma 12:1-21.

Basso DM, Fisher LC, Anderson AJ, Jakeman LB, McTigue DM, Popovich PG (2006) Basso Mouse Scale for locomotion detects differences in recovery after spinal cord injury in five common mouse strains. Journal of neurotrauma 23:635659.

Bauer NG, Richter-Landsberg C, Ffrench-Constant C (2009) Role of the oligodendroglial cytoskeleton in differentiation and myelination. Glia 57:16911705 . 
Baumann N, Pham-Dinh D (2001) Biology of oligodendrocyte and myelin in the mammalian central nervous system. Physiological reviews 81:871-927.

Beare JE, Morehouse JR, DeVries WH, Enzmann GU, Burke DA, Magnuson DS, Whittemore SR (2009) Gait analysis in normal and spinal contused mice using the TreadScan system. Journal of neurotrauma 26:2045-2056.

Ben-Hur T, Goldman SA (2008) Prospects of cell therapy for disorders of myelin. Annals of the New York Academy of Sciences 1142:218-249.

Bester M, Petracca M, Inglese M (2014) Neuroimaging of multiple sclerosis, acute disseminated encephalomyelitis, and other demyelinating diseases. Seminars in roentgenology 49:76-85.

Black JA, Kocsis JD, Waxman SG (1990) lon channel organization of the myelinated fiber. Trends in neurosciences 13:48-54.

Blakemore WF (1974) Pattern of remyelination in the CNS. Nature 249:577-578.

Blakemore WF (1982) Ethidium bromide induced demyelination in the spinal cord of the cat. Neuropathology and applied neurobiology 8:365-375. 
Blakemore WF (1984) The response of oligodendrocytes to chemical injury. Acta neurologica Scandinavica Supplementum 100:33-38.

Blakemore WF (1995) Cross talk between the immune system and the nervous system in response to injury: implications for regeneration. Human \& experimental toxicology 14:615-616.

Blakemore WF (2005) The case for a central nervous system (CNS) origin for the Schwann cells that remyelinate CNS axons following concurrent loss of oligodendrocytes and astrocytes. Neuropathology and applied neurobiology 31:110.

Blakemore WF, Franklin RJ (2008) Remyelination in experimental models of toxin-induced demyelination. Current topics in microbiology and immunology 318:193-212.

Blight AR (1983) Cellular morphology of chronic spinal cord injury in the cat: analysis of myelinated axons by line-sampling. Neuroscience 10:521-543.

Blomqvist M, Gieselmann V, Mansson JE (2011) Accumulation of lysosulfatide in the brain of arylsulfatase A-deficient mice. Lipids in health and disease 10:28. 
Bradl M, Lassmann H (2010) Oligodendrocytes: biology and pathology. Acta neuropathologica 119:37-53.

Bramow S, Frischer JM, Lassmann H, Koch-Henriksen N, Lucchinetti CF, Sorensen PS, Laursen H (2010) Demyelination versus remyelination in progressive multiple sclerosis.

Brain : a journal of neurology 133:2983-2998.

Brustle O, Jones KN, Learish RD, Karram K, Choudhary K, Wiestler OD, Duncan ID, McKay RD (1999) Embryonic stem cell-derived glial precursors: a source of myelinating transplants. Science (New York, NY) 285:754-756.

Bunge MB, Bunge RP, Ris H (1961) Ultrastructural study of remyelination in an experimental lesion in adult cat spinal cord. The Journal of biophysical and biochemical cytology 10:67-94.

Buntinx M, Vanderlocht J, Hellings N, Vandenabeele F, Lambrichts I, Raus J, Ameloot M, Stinissen P, Steels P (2003) Characterization of three human oligodendroglial cell lines as a model to study oligodendrocyte injury: morphology and oligodendrocyte-specific gene expression. Journal of neurocytology 32:2538. 
Buschmann JP, Berger K, Awad H, Clarner T, Beyer C, Kipp M (2012) Inflammatory response and chemokine expression in the white matter corpus callosum and gray matter cortex region during cuprizone-induced demyelination. Journal of molecular neuroscience : MN 48:66-76.

Cao Q, He Q, Wang Y, Cheng X, Howard RM, Zhang Y, DeVries WH, Shields CB, Magnuson DS, Xu XM, Kim DH, Whittemore SR (2010) Transplantation of ciliary neurotrophic factor-expressing adult oligodendrocyte precursor cells promotes remyelination and functional recovery after spinal cord injury. The Journal of neuroscience : the official journal of the Society for Neuroscience 30:2989-3001.

Cao Q, Xu XM, Devries WH, Enzmann GU, Ping P, Tsoulfas P, Wood PM, Bunge MB, Whittemore SR (2005) Functional recovery in traumatic spinal cord injury after transplantation of multineurotrophin-expressing glial-restricted precursor cells. The Journal of neuroscience : the official journal of the Society for Neuroscience 25:6947-6957.

Carthew RW, Sontheimer EJ (2009) Origins and Mechanisms of miRNAs and siRNAs. Cell 136:642-655. 
Casaccia-Bonnefil P, Liu A (2003) Relationship between cell cycle molecules and onset of oligodendrocyte differentiation. Journal of neuroscience research 72:111.

Cenik ES, Zamore PD (2011) Argonaute proteins. Current biology : CB 21:R446449.

Chen F, Hu SJ (2012) Effect of microRNA-34a in cell cycle, differentiation, and apoptosis: a review. Journal of biochemical and molecular toxicology 26:79-86.

Chen K, Rajewsky N (2007) The evolution of gene regulation by transcription factors and microRNAs. Nature reviews Genetics 8:93-103.

Chernoff GF (1981) Shiverer: an autosomal recessive mutant mouse with myelin deficiency. The Journal of heredity 72:128.

Ciesla M, Skrzypek K, Kozakowska M, Loboda A, Jozkowicz A, Dulak J (2011) MicroRNAs as biomarkers of disease onset. Analytical and bioanalytical chemistry 401:2051-2061.

Constantinescu CS, Farooqi N, O'Brien K, Gran B (2011) Experimental autoimmune encephalomyelitis (EAE) as a model for multiple sclerosis (MS). British journal of pharmacology 164:1079-1106. 
Crang AJ, Blakemore WF (1991) Remyelination of demyelinated rat axons by transplanted mouse oligodendrocytes. Glia 4:305-313.

Debs R, Froissart R, Aubourg P, Papeix C, Douillard C, Degos B, Fontaine B, Audoin B, Lacour A, Said G, Vanier MT, Sedel F (2013) Krabbe disease in adults: phenotypic and genotypic update from a series of 11 cases and a review. Journal of inherited metabolic disease 36:859-868.

Desjardins P, Frost E, Morais R (1985) Ethidium bromide-induced loss of mitochondrial DNA from primary chicken embryo fibroblasts. Molecular and cellular biology 5:1163-1169.

Druz A, Betenbaugh M, Shiloach J (2012) Glucose depletion activates mmu-miR466h-5p expression through oxidative stress and inhibition of histone deacetylation. Nucleic acids research 40:7291-7302.

Druz A, Chu C, Majors B, Santuary R, Betenbaugh M, Shiloach J (2011) A novel microRNA mmu-miR-466h affects apoptosis regulation in mammalian cells. Biotechnology and bioengineering 108:1651-1661. 
Dubois J, Dehaene-Lambertz G, Kulikova S, Poupon C, Huppi PS, Hertz-Pannier $L$ (2013) The early development of brain white matter: A review of imaging studies in fetuses, newborns and infants. Neuroscience.

Dugas JC, Cuellar TL, Scholze A, Ason B, Ibrahim A, Emery B, Zamanian JL, Foo LC, McManus MT, Barres BA (2010) Dicer1 and miR-219 Are required for normal oligodendrocyte differentiation and myelination. Neuron 65:597-611.

Eisen MB, Spellman PT, Brown PO, Botstein D (1998) Cluster analysis and display of genome-wide expression patterns. Proceedings of the National Academy of Sciences of the United States of America 95:14863-14868.

Ellison JA, de Vellis J (1994) Platelet-derived growth factor receptor is expressed by cells in the early oligodendrocyte lineage. Journal of neuroscience research 37:116-128.

Eltorai MI (2003) Spinal Cord Medicine: Principles and Practice. Chapter One: The History of Spinal Cord Medicine. New York: Demos Medical Publishing.

Emdad L, D'Souza SL, Kothari HP, Qadeer ZA, Germano IM (2012) Efficient differentiation of human embryonic and induced pluripotent stem cells into functional astrocytes. Stem cells and development 21:404-410. 
Emery B (2010) Transcriptional and post-transcriptional control of CNS myelination. Current opinion in neurobiology 20:601-607.

Engesser-Cesar C, Anderson AJ, Basso DM, Edgerton VR, Cotman CW (2005) Voluntary wheel running improves recovery from a moderate spinal cord injury. Journal of neurotrauma 22:157-171.

Engesser-Cesar C, Ichiyama RM, Nefas AL, Hill MA, Edgerton VR, Cotman CW, Anderson AJ (2007) Wheel running following spinal cord injury improves locomotor recovery and stimulates serotonergic fiber growth. The European journal of neuroscience 25:1931-1939.

England JD, Gamboni F, Levinson SR, Finger TE (1990) Changed distribution of sodium channels along demyelinated axons. Proceedings of the National Academy of Sciences of the United States of America 87:6777-6780.

Enzmann GU, Benton RL, Talbott JF, Cao Q, Whittemore SR (2006) Functional considerations of stem cell transplantation therapy for spinal cord repair. Journal of neurotrauma 23:479-495.

Esau C, Davis S, Murray SF, Yu XX, Pandey SK, Pear M, Watts L, Booten SL, Graham M, McKay R, Subramaniam A, Propp S, Lollo BA, Freier S, Bennett CF, 
Bhanot S, Monia BP (2006) miR-122 regulation of lipid metabolism revealed by in vivo antisense targeting. Cell metabolism 3:87-98.

Evans C, Beland SG, Kulaga S, Wolfson C, Kingwell E, Marriott J, Koch M, Makhani N, Morrow S, Fisk J, Dykeman J, Jette N, Pringsheim T, Marrie RA (2013) Incidence and prevalence of multiple sclerosis in the Americas: a systematic review. Neuroepidemiology 40:195-210.

Fancy SP, Baranzini SE, Zhao C, Yuk DI, Irvine KA, Kaing S, Sanai N, Franklin RJ, Rowitch DH (2009) Dysregulation of the Wnt pathway inhibits timely myelination and remyelination in the mammalian CNS. Genes \& development 23:1571-1585.

Fehlings MG, Vaccaro AR, Boakye M (2013) Essentials of spinal cord injury: Basic research to clinical practice. New York: Thieme.

Fluharty AL (2006) Arylsulfatase A Deficiency. Seattle, WA: GeneReviews ${ }^{\mathrm{TM}}$ [Internet].

Flynt AS, Lai EC (2008) Biological principles of microRNA-mediated regulation: shared themes amid diversity. Nature reviews Genetics 9:831-842. 
Fouad K, Metz GA, Merkler D, Dietz V, Schwab ME (2000) Treadmill training in incomplete spinal cord injured rats. Behavioural brain research 115:107-113.

Franklin RJ, Bayley SA, Blakemore WF (1996) Transplanted CG4 cells (an oligodendrocyte progenitor cell line) survive, migrate, and contribute to repair of areas of demyelination in X-irradiated and damaged spinal cord but not in normal spinal cord. Experimental neurology 137:263-276.

Franklin RJ, Ffrench-Constant C (2008) Remyelination in the CNS: from biology to therapy. Nat Rev Neurosci 9:839-855.

Frederick TJ, Min J, Altieri SC, Mitchell NE, Wood TL (2007) Synergistic induction of cyclin D1 in oligodendrocyte progenitor cells by IGF-I and FGF-2 requires differential stimulation of multiple signaling pathways. Glia 55:10111022.

Frederick TJ, Wood TL (2004) IGF-I and FGF-2 coordinately enhance cyclin D1 and cyclin E-cdk2 association and activity to promote G1 progression in oligodendrocyte progenitor cells. Molecular and cellular neurosciences 25:480492. 
Fulk GD, Behrman A, Schmitz T (2007) Traumatic Spinal Cord Injury In:

O'Sullivan S, Schmitz T, eds. Physical Rehabilitation Assessment and Treatment 5th ed. Philadelphia, PA: F. A. Davis.

Fuller ML, DeChant AK, Rothstein B, Caprariello A, Wang R, Hall AK, Miller RH (2007) Bone morphogenetic proteins promote gliosis in demyelinating spinal cord lesions. Annals of neurology 62:288-300.

Fushimi S, Shirabe T (2002) The reaction of glial progenitor cells in remyelination following ethidium bromide-induced demyelination in the mouse spinal cord. Neuropathology : official journal of the Japanese Society of Neuropathology 22:233-242.

Fushimi S, Shirabe T (2004) Expression of insulin-like growth factors in remyelination following ethidium bromide-induced demyelination in the mouse spinal cord. Neuropathology : official journal of the Japanese Society of Neuropathology 24:208-218.

Gao L, Miller RH (2006) Specification of optic nerve oligodendrocyte precursors by retinal ganglion cell axons. The Journal of neuroscience : the official journal of the Society for Neuroscience 26:7619-7628. 
Ge Y (2006) Multiple sclerosis: the role of MR imaging. AJNR American journal of neuroradiology 27:1165-1176.

Gensert JM, Goldman JE (1997) Endogenous progenitors remyelinate demyelinated axons in the adult CNS. Neuron 19:197-203.

Ghelani HS, Rachchh MA, Gokani RH (2012) MicroRNAs as newer therapeutic targets: A big hope from a tiny player. Journal of pharmacology \& pharmacotherapeutics 3:217-227.

Giger RJ, Venkatesh K, Chivatakarn O, Raiker SJ, Robak L, Hofer T, Lee H, Rader C (2008) Mechanisms of CNS myelin inhibition: evidence for distinct and neuronal cell type specific receptor systems. Restorative neurology and neuroscience 26:97-115.

Gledhill RF, Harrison BM, McDonald WI (1973) Demyelination and remyelination after acute spinal cord compression. Experimental neurology 38:472-487.

Gledhill RF, McDonald WI (1977) Morphological characteristics of central demyelination and remyelination: a single-fiber study. Annals of neurology 1:552560. 
Goldman SA, Windrem MS (2006) Cell replacement therapy in neurological disease. Philosophical transactions of the Royal Society of London Series B, Biological sciences 361:1463-1475.

Gordon N (2001) Canavan disease: a review of recent developments. European journal of paediatric neurology : EJPN : official journal of the European Paediatric Neurology Society 5:65-69.

Graca DL, Blakemore WF (1986) Delayed remyelination in rat spinal cord following ethidium bromide injection. Neuropathology and applied neurobiology 12:593-605.

Gregson NA, Hall SM (1973) A quantitative analysis of the effects of the intraneural injection of lysophosphatidyl choline. Journal of cell science 13:257277.

Guest JD, Hiester ED, Bunge RP (2005) Demyelination and Schwann cell responses adjacent to injury epicenter cavities following chronic human spinal cord injury. Experimental neurology 192:384-393.

Han SS, Liu Y, Tyler-Polsz C, Rao MS, Fischer I (2004) Transplantation of glialrestricted precursor cells into the adult spinal cord: survival, glial-specific differentiation, and preferential migration in white matter. Glia 45:1-16. 
Harkema S, Gerasimenko Y, Hodes J, Burdick J, Angeli C, Chen Y, Ferreira C, Willhite A, Rejc E, Grossman RG, Edgerton VR (2011) Effect of epidural stimulation of the lumbosacral spinal cord on voluntary movement, standing, and assisted stepping after motor complete paraplegia: a case study. Lancet $377: 1938-1947$.

Hayakawa T, Noda M, Yasuda K, Yorifuji H, Taniguchi S, Miwa I, Sakura H, Terauchi Y, Hayashi J, Sharp GW, Kanazawa Y, Akanuma Y, Yazaki Y, Kadowaki T (1998) Ethidium bromide-induced inhibition of mitochondrial gene transcription suppresses glucose-stimulated insulin release in the mouse pancreatic beta-cell line betaHC9. The Journal of biological chemistry 273:20300-20307.

Hayashi J, Tanaka M, Sato W, Ozawa T, Yonekawa H, Kagawa Y, Ohta S (1990) Effects of ethidium bromide treatment of mouse cells on expression and assembly of nuclear-coded subunits of complexes involved in the oxidative phosphorylation. Biochemical and biophysical research communications 167:216-221.

He X, Yu Y, Awatramani R, Lu QR (2012) Unwrapping myelination by microRNAs. The Neuroscientist : a review journal bringing neurobiology, neurology and psychiatry 18:45-55. 
He Y, Casaccia-Bonnefil P (2008) The Yin and Yang of YY1 in the nervous system. Journal of neurochemistry 106:1493-1502.

He Y, Dupree J, Wang J, Sandoval J, Li J, Liu H, Shi Y, Nave KA, CasacciaBonnefil P (2007) The transcription factor Yin Yang 1 is essential for oligodendrocyte progenitor differentiation. Neuron 55:217-230.

He Y, Sandoval J, Casaccia-Bonnefil P (2007) Events at the transition between cell cycle exit and oligodendrocyte progenitor differentiation: the role of HDAC and YY1. Neuron glia biology 3:221-231.

Hibbits N, Yoshino J, Le TQ, Armstrong RC (2012) Astrogliosis during acute and chronic cuprizone demyelination and implications for remyelination. ASN neuro 4:393-408.

Hill CE, Proschel C, Noble M, Mayer-Proschel M, Gensel JC, Beattie MS, Bresnahan JC (2004) Acute transplantation of glial-restricted precursor cells into spinal cord contusion injuries: survival, differentiation, and effects on lesion environment and axonal regeneration. Experimental neurology 190:289-310.

Hill RL, Zhang YP, Burke DA, Devries WH, Zhang Y, Magnuson DS, Whittemore SR, Shields CB (2009) Anatomical and functional outcomes following a precise, 
graded, dorsal laceration spinal cord injury in C57BL/6 mice. Journal of neurotrauma 26:1-15.

Hinks GL, Chari DM, O'Leary MT, Zhao C, Keirstead HS, Blakemore WF, Franklin RJ (2001) Depletion of endogenous oligodendrocyte progenitors rather than increased availability of survival factors is a likely explanation for enhanced survival of transplanted oligodendrocyte progenitors in X-irradiated compared to normal CNS. Neuropathology and applied neurobiology 27:59-67.

Hodes ME, Pratt VM, Dlouhy SR (1993) Genetics of Pelizaeus-Merzbacher disease. Developmental neuroscience 15:383-394.

Hofstetter CP, Holmstrom NA, Lilja JA, Schweinhardt P, Hao J, Spenger C, Wiesenfeld-Hallin Z, Kurpad SN, Frisen J, Olson L (2005) Allodynia limits the usefulness of intraspinal neural stem cell grafts; directed differentiation improves outcome. Nature neuroscience 8:346-353.

Horner PJ, Gage FH (2000) Regenerating the damaged central nervous system. Nature 407:963-970.

Houle JD, Cote MP (2013) Axon regeneration and exercise-dependent plasticity after spinal cord injury. Annals of the New York Academy of Sciences 1279:154163. 
Hu G, Drescher KM, Chen XM (2012) Exosomal miRNAs: Biological Properties and Therapeutic Potential. Frontiers in genetics 3:56.

Hu W, Coller J (2012) What comes first: translational repression or mRNA degradation? The deepening mystery of microRNA function. Cell research 22:1322-1324.

Huang H, Zhao XF, Zheng K, Qiu M (2013) Regulation of the timing of oligodendrocyte differentiation: mechanisms and perspectives. Neuroscience bulletin 29:155-164.

Huang V, Place RF, Portnoy V, Wang J, Qi Z, Jia Z, Yu A, Shuman M, Yu J, Li LC (2012) Upregulation of Cyclin B1 by miRNA and its implications in cancer. Nucleic acids research 40:1695-1707.

lester A, Vignola S, Callegarini L, Gimelli G, Alpigiani MG (1996) [18q syndrome with deficiency of myelin basic protein (MBP)]. La Pediatria medica e chirurgica : Medical and surgical pediatrics 18:201-205.

Jeffery ND, Blakemore WF (1995) Remyelination of mouse spinal cord axons demyelinated by local injection of lysolecithin. Journal of neurocytology 24:775781. 
John B, Enright AJ, Aravin A, Tuschl T, Sander C, Marks DS (2004) Human MicroRNA targets. PLoS biology 2:e363.

Jones RE, Chou Y, Young A, Mass M, Vandenbark A, Offner H, Bourdette D (1995) T cells with encephalitogenic potential from multiple sclerosis patients and Lewis rats fail to induce disease in SCID mice following intracisternal injection. Journal of neuroimmunology 56:119-126.

Jordan L (1986) Initiation of Locomotion from the Mammalian Brainstem. In: Grillner, S., Stein, P. S. G., Stuart, D. G., Forssberg, H., and Herman, R. M., (Eds.), Neurobiology of Vertebrate Locomotion. . London: Macmillan Press.

Jordan LM (1991) Brainstem and Spinal Cord Mechanisms for the Initiation of Locomotion. In: Shimamura, M., Grillner, S., and Edgerton, V. R., (Eds.), Neurobiological Basis of Human Locomotion. . Tokyo, Japan.

Joubert L, Foucault I, Sagot Y, Bernasconi L, Duval F, Alliod C, Frossard MJ, Pescini Gobert R, Curchod ML, Salvat C, Nichols A, Pouly S, Rommel C, Roach A, Hooft van Huijsduijnen R (2010) Chemical inducers and transcriptional markers of oligodendrocyte differentiation. Journal of neuroscience research 88:2546-2557. 
Jung M, Kramer E, Grzenkowski M, Tang K, Blakemore W, Aguzzi A, Khazaie K, Chlichlia K, von Blankenfeld G, Kettenmann H, et al. (1995) Lines of murine oligodendroglial precursor cells immortalized by an activated neu tyrosine kinase show distinct degrees of interaction with axons in vitro and in vivo. The European journal of neuroscience 7:1245-1265.

Junker A, Krumbholz M, Eisele S, Mohan H, Augstein F, Bittner R, Lassmann H, Wekerle H, Hohlfeld R, Meinl E (2009) MicroRNA profiling of multiple sclerosis lesions identifies modulators of the regulatory protein CD47. Brain : a journal of neurology 132:3342-3352.

Kakulas BA (1999) A review of the neuropathology of human spinal cord injury with emphasis on special features. The journal of spinal cord medicine 22:119124.

Kang SM, Cho MS, Seo H, Yoon CJ, Oh SK, Choi YM, Kim DW (2007) Efficient induction of oligodendrocytes from human embryonic stem cells. Stem cells (Dayton, Ohio) 25:419-424.

Karimi-Abdolrezaee S, Eftekharpour E, Wang J, Morshead CM, Fehlings MG (2006) Delayed transplantation of adult neural precursor cells promotes remyelination and functional neurological recovery after spinal cord injury. The 
Journal of neuroscience : the official journal of the Society for Neuroscience 26:3377-3389.

Kasinski AL, Slack FJ (2011) Epigenetics and genetics. MicroRNAs en route to the clinic: progress in validating and targeting microRNAs for cancer therapy. Nature reviews Cancer 11:849-864.

Kefas B, Floyd DH, Comeau L, Frisbee A, Dominguez C, Dipierro CG, Guessous F, Abounader R, Purow B (2013) A miR-297/hypoxia/DGK-alpha axis regulating glioblastoma survival. Neuro-oncology 15:1652-1663.

Kelley RE (2006) Ischemic demyelination. Neurological research 28:334-340.

Kipp M, Victor M, Martino G, Franklin RJ (2012) Endogeneous remyelination: findings in human studies. CNS \& neurological disorders drug targets 11:598609.

Kirshblum SC, O'Connor KC (1998) Predicting neurologic recovery in traumatic cervical spinal cord injury. Archives of physical medicine and rehabilitation 79:1456-1466.

Klein C, Kramer EM, Cardine AM, Schraven B, Brandt R, Trotter J (2002) Process outgrowth of oligodendrocytes is promoted by interaction of fyn kinase 
with the cytoskeletal protein tau. The Journal of neuroscience : the official journal of the Society for Neuroscience 22:698-707.

Kohlschutter A, Eichler F (2011) Childhood leukodystrophies: a clinical perspective. Expert review of neurotherapeutics 11:1485-1496.

Kohoutek J (2009) P-TEFb- the final frontier. Cell division 4:19.

Kotter MR, Li WW, Zhao C, Franklin RJ (2006) Myelin impairs CNS remyelination by inhibiting oligodendrocyte precursor cell differentiation. The Journal of neuroscience : the official journal of the Society for Neuroscience 26:328-332.

Krageloh-Mann I, Groeschel S, Kehrer C, Opherk K, Nagele T, Handgretinger R, Muller I (2013) Juvenile metachromatic leukodystrophy 10 years post transplant compared with a non-transplanted cohort. Bone marrow transplantation 48:369375.

Kuerzi J, Brown EH, Shum-Siu A, Siu A, Burke D, Morehouse J, Smith RR, Magnuson DS (2010) Task-specificity vs. ceiling effect: step-training in shallow water after spinal cord injury. Experimental neurology 224:178-187. 
Kuypers NJ, James KT, Enzmann GU, Magnuson DS, Whittemore SR (2013) Functional consequences of ethidium bromide demyelination of the mouse ventral spinal cord. Experimental neurology 247:615-622.

Kuzmin A, Han Z, Golding MC, Mann MR, Latham KE, Varmuza S (2008) The PcG gene Sfmbt2 is paternally expressed in extraembryonic tissues. Gene expression patterns : GEP 8:107-116.

La Piana R, Vanderver A, van der Knaap M, Roux L, Tampieri D, Brais B, Bernard G (2012) Adult-onset vanishing white matter disease due to a novel EIF2B3 mutation. Archives of neurology 69:765-768.

Lasiene J, Shupe L, Perlmutter S, Horner P (2008) No evidence for chronic demyelination in spared axons after spinal cord injury in a mouse. The Journal of neuroscience : the official journal of the Society for Neuroscience 28:3887-3896.

Lau P, Verrier JD, Nielsen JA, Johnson KR, Notterpek L, Hudson LD (2008) Identification of dynamically regulated microRNA and mRNA networks in developing oligodendrocytes. The Journal of neuroscience : the official journal of the Society for Neuroscience 28:11720-11730. 
Laursen LS, Chan CW, Ffrench-Constant C (2011) Translation of myelin basic protein mRNA in oligodendrocytes is regulated by integrin activation and hnRNPK. The Journal of cell biology 192:797-811.

Lee Y, Morrison BM, Li Y, Lengacher S, Farah MH, Hoffman PN, Liu Y, Tsingalia A, Jin L, Zhang PW, Pellerin L, Magistretti PJ, Rothstein JD (2012) Oligodendroglia metabolically support axons and contribute to neurodegeneration. Nature 487:443-448.

Leone V, D'Angelo D, Ferraro A, Pallante P, Rubio I, Santoro M, Croce CM, Fusco A (2011) A TSH-CREB1-microRNA loop is required for thyroid cell growth. Molecular endocrinology (Baltimore, Md) 25:1819-1830.

Levine JM, Reynolds R, Fawcett JW (2001) The oligodendrocyte precursor cell in health and disease. Trends in neurosciences 24:39-47.

Li C, Xiao L, Liu X, Yang W, Shen W, Hu C, Yang G, He C (2013) A functional role of NMDA receptor in regulating the differentiation of oligodendrocyte precursor cells and remyelination. Glia 61:732-749.

Li R, Messing A, Goldman JE, Brenner M (2002) GFAP mutations in Alexander disease. International journal of developmental neuroscience : the official journal of the International Society for Developmental Neuroscience 20:259-268. 
Lin ST, Fu YH (2009) miR-23 regulation of lamin B1 is crucial for oligodendrocyte development and myelination. Disease models \& mechanisms 2:178-188.

Linden RD, Zhang YP, Burke DA, Hunt MA, Harpring JE, Shields CB (1999) Magnetic motor evoked potential monitoring in the rat. Journal of neurosurgery 91:205-210.

Liu NK, Wang XF, Lu QB, Xu XM (2009) Altered microRNA expression following traumatic spinal cord injury. Experimental neurology 219:424-429.

Louis JC, Magal E, Muir D, Manthorpe M, Varon S (1992) CG-4, a new bipotential glial cell line from rat brain, is capable of differentiating in vitro into either mature oligodendrocytes or type-2 astrocytes. Journal of neuroscience research 31:193-204.

Lovas G, Szilagyi N, Majtenyi K, Palkovits M, Komoly S (2000) Axonal changes in chronic demyelinated cervical spinal cord plaques. Brain : a journal of neurology 123 ( Pt 2):308-317.

Loy DN, Magnuson DS, Zhang YP, Onifer SM, Mills MD, Cao QL, Darnall JB, Fajardo LC, Burke DA, Whittemore SR (2002) Functional redundancy of ventral spinal locomotor pathways. The Journal of neuroscience : the official journal of the Society for Neuroscience 22:315-323. 
Loy DN, Talbott JF, Onifer SM, Mills MD, Burke DA, Dennison JB, Fajardo LC, Magnuson DS, Whittemore SR (2002) Both dorsal and ventral spinal cord pathways contribute to overground locomotion in the adult rat. Experimental neurology 177:575-580.

Lu J, He ML, Wang L, Chen Y, Liu X, Dong Q, Chen YC, Peng Y, Yao KT, Kung HF, Li XP (2011) MiR-26a inhibits cell growth and tumorigenesis of nasopharyngeal carcinoma through repression of EZH2. Cancer research 71:225-233.

Magnuson DS, Lovett R, Coffee C, Gray R, Han Y, Zhang YP, Burke DA (2005) Functional consequences of lumbar spinal cord contusion injuries in the adult rat. Journal of neurotrauma 22:529-543.

Magnuson DS, Trinder TC, Zhang YP, Burke D, Morassutti DJ, Shields CB (1999) Comparing deficits following excitotoxic and contusion injuries in the thoracic and lumbar spinal cord of the adult rat. Experimental neurology 156:191204.

Malhas A, Saunders NJ, Vaux DJ (2010) The nuclear envelope can control gene expression and cell cycle progression via miRNA regulation. Cell cycle (Georgetown, Tex) 9:531-539. 
Mason JL, Langaman C, Morell P, Suzuki K, Matsushima GK (2001) Episodic demyelination and subsequent remyelination within the murine central nervous system: changes in axonal calibre. Neuropathology and applied neurobiology 27:50-58.

Matalon RM-M, K. (1999) Canavan Disease. Seattle, WA: GeneReviews ${ }^{\mathrm{TM}}$ [Internet].

Matsukawa T, Wang X, Liu R, Wortham NC, Onuki Y, Kubota A, Hida A, Kowa H, Fukuda Y, Ishiura H, Mitsui J, Takahashi Y, Aoki S, Takizawa S, Shimizu J, Goto J, Proud CG, Tsuji S (2011) Adult-onset leukoencephalopathies with vanishing white matter with novel missense mutations in EIF2B2, EIF2B3, and EIF2B5. Neurogenetics 12:259-261.

Matsushima GK, Morell P (2001) The neurotoxicant, cuprizone, as a model to study demyelination and remyelination in the central nervous system. Brain pathology (Zurich, Switzerland) 11:107-116.

Mattes J, Collison A, Plank M, Phipps S, Foster PS (2009) Antagonism of microRNA-126 suppresses the effector function of $\mathrm{TH} 2$ cells and the development of allergic airways disease. Proceedings of the National Academy of Sciences of the United States of America 106:18704-18709. 
Matute C (2011) Glutamate and ATP signalling in white matter pathology. Journal of anatomy 219:53-64.

McKinley W, Santos K, Meade M, Brooke K (2007) Incidence and outcomes of spinal cord injury clinical syndromes. The journal of spinal cord medicine 30:215224.

Messing A, Brenner M, Feany MB, Nedergaard M, Goldman JE (2012) Alexander disease. The Journal of neuroscience : the official journal of the Society for Neuroscience 32:5017-5023.

Miller DM, Rudick RA, Cutter G, Baier M, Fischer JS (2000) Clinical significance of the multiple sclerosis functional composite: relationship to patient-reported quality of life. Archives of neurology 57:1319-1324.

Morell PQ, R. H. (1999) Characteristic Composition of Myelin. In: Basic Neurochemistry: Molecular, Cellular and Medical Aspects. 6th edition. . Philadelphia: Lippincott-Raven.

Nave K-A (2010) Myelination and the trophic support of long axons. Nat Rev Neurosci 11:275-283. 
Nichols NL, Punzo AM, Duncan ID, Mitchell GS, Johnson RA (2013) Cervical spinal demyelination with ethidium bromide impairs respiratory (phrenic) activity and forelimb motor behavior in rats. Neuroscience 229:77-87.

Nistor GI, Totoiu MO, Haque N, Carpenter MK, Keirstead HS (2005) Human embryonic stem cells differentiate into oligodendrocytes in high purity and myelinate after spinal cord transplantation. Glia 49:385-396.

Noga BR, Kriellaars DJ, Brownstone RM, Jordan LM (2003) Mechanism for activation of locomotor centers in the spinal cord by stimulation of the mesencephalic locomotor region. Journal of neurophysiology 90:1464-1478.

Noga BR, Kriellaars DJ, Jordan LM (1991) The effect of selective brainstem or spinal cord lesions on treadmill locomotion evoked by stimulation of the mesencephalic or pontomedullary locomotor regions. The Journal of neuroscience : the official journal of the Society for Neuroscience 11:1691-1700.

Norenberg MD, Smith J, Marcillo A (2004) The pathology of human spinal cord injury: defining the problems. Journal of neurotrauma 21:429-440.

Novak N, Bar V, Sabanay H, Frechter S, Jaegle M, Snapper SB, Meijer D, Peles E (2011) N-WASP is required for membrane wrapping and myelination by Schwann cells. The Journal of cell biology 192:243-250. 
Ohri SS, Maddie MA, Zhao Y, Qiu MS, Hetman M, Whittemore SR (2011) Attenuating the endoplasmic reticulum stress response improves functional recovery after spinal cord injury. Glia 59:1489-1502.

Onifer SM, Rabchevsky AG, Scheff SW (2007) Rat models of traumatic spinal cord injury to assess motor recovery. ILAR journal / National Research Council, Institute of Laboratory Animal Resources 48:385-395.

Ono K, Yasui Y, Rutishauser U, Miller RH (1997) Focal ventricular origin and migration of oligodendrocyte precursors into the chick optic nerve. Neuron 19:283-292.

Ortega JA, Radonjic NV, Zecevic N (2013) Sonic hedgehog promotes generation and maintenance of human forebrain Olig2 progenitors. Frontiers in cellular neuroscience 7:254.

Pareyson D, Fancellu R, Mariotti C, Romano S, Salmaggi A, Carella F, Girotti F, Gattellaro G, Carriero MR, Farina L, Ceccherini I, Savoiardo M (2008) Adultonset Alexander disease: a series of eleven unrelated cases with review of the literature. Brain : a journal of neurology 131:2321-2331. 
Patrikios P, Stadelmann C, Kutzelnigg A, Rauschka H, Schmidbauer M, Laursen H, Sorensen PS, Bruck W, Lucchinetti C, Lassmann H (2006) Remyelination is extensive in a subset of multiple sclerosis patients. Brain : a journal of neurology 129:3165-3172.

Pawitan Y, Michiels S, Koscielny S, Gusnanto A, Ploner A (2005) False discovery rate, sensitivity and sample size for microarray studies. Bioinformatics (Oxford, England) 21:3017-3024.

Penderis J, Shields SA, Franklin RJ (2003) Impaired remyelination and depletion of oligodendrocyte progenitors does not occur following repeated episodes of focal demyelination in the rat central nervous system. Brain : a journal of neurology 126:1382-1391.

Peng J, Zhu Y, Milton JT, Price DH (1998) Identification of multiple cyclin subunits of human P-TEFb. Genes \& development 12:755-762.

Perlman SJ, Mar S (2012) Leukodystrophies. Advances in experimental medicine and biology 724:154-171.

Poduslo SE, Jang Y (1984) Myelin development in infant brain. Neurochemical research 9:1615-1626.

Poeppel P, Habetha M, Marcao A, Bussow H, Berna L, Gieselmann V (2005) Missense mutations as a cause of metachromatic leukodystrophy. Degradation 
of arylsulfatase A in the endoplasmic reticulum. The FEBS journal 272:11791188.

Price DH (2000) P-TEFb, a cyclin-dependent kinase controlling elongation by RNA polymerase II. Molecular and cellular biology 20:2629-2634.

Pringle NP, Mudhar HS, Collarini EJ, Richardson WD (1992) PDGF receptors in the rat CNS: during late neurogenesis, PDGF alpha-receptor expression appears to be restricted to glial cells of the oligodendrocyte lineage. Development (Cambridge, England) 115:535-551.

Pronk JC, van Kollenburg B, Scheper GC, van der Knaap MS (2006) Vanishing white matter disease: a review with focus on its genetics. Mental retardation and developmental disabilities research reviews 12:123-128.

Quilliam TA (1958) Growth changes in sensory nerve fibre aggregates undergoing remyelination. Journal of anatomy 92:383-398.

Rabinowits G, Gercel-Taylor C, Day JM, Taylor DD, Kloecker GH (2009) Exosomal microRNA: a diagnostic marker for lung cancer. Clinical lung cancer $10: 42-46$. 
Radtke C, Akiyama Y, Brokaw J, Lankford KL, Wewetzer K, Fodor WL, Kocsis JD (2004) Remyelination of the nonhuman primate spinal cord by transplantation of $\mathrm{H}$-transferase transgenic adult pig olfactory ensheathing cells. FASEB journal : official publication of the Federation of American Societies for Experimental Biology 18:335-337.

Readhead C, Hood L (1990) The dysmyelinating mouse mutations shiverer (shi) and myelin deficient (shimld). Behavior genetics 20:213-234.

Roy NS, Wang S, Harrison-Restelli C, Benraiss A, Fraser RA, Gravel M, Braun PE, Goldman SA (1999) Identification, isolation, and promoter-defined separation of mitotic oligodendrocyte progenitor cells from the adult human subcortical white matter. The Journal of neuroscience : the official journal of the Society for Neuroscience 19:9986-9995.

Rusk N (2008) When microRNAs activate translation. Nat Meth 5:122-123.

Sadovnick AD, Tuokko H, Applegarth DA, Toone JR, Hadjistavropoulos T, Beattie BL (1993) The differential diagnosis of adult onset metachromatic leukodystrophy and early onset familial Alzheimer disease in an Alzheimer clinic population. The Canadian journal of neurological sciences Le journal canadien des sciences neurologiques 20:312-318. 
Sakai N (2009) Pathogenesis of leukodystrophy for Krabbe disease: molecular mechanism and clinical treatment. Brain \& development 31:485-487.

Sampaio-Baptista C, Khrapitchev AA, Foxley S, Schlagheck T, Scholz J, Jbabdi S, Deluca GC, Miller KL, Taylor A, Thomas N, Kleim J, Sibson NR, Bannerman D, Johansen-Berg H (2013) Motor skill learning induces changes in white matter microstructure and myelination. The Journal of neuroscience : the official journal of the Society for Neuroscience 33:19499-19503.

Sato D, Callegaro D, Lana-Peixoto MA, Fujihara K (2012) Treatment of neuromyelitis optica: an evidence based review. Arquivos de neuro-psiquiatria 70:59-66.

Schneider GS (2010) Anterior spinal cord syndrome after initiation of treatment with atenolol. The Journal of emergency medicine 38:e49-52.

Schucht P, Raineteau O, Schwab ME, Fouad K (2002) Anatomical correlates of locomotor recovery following dorsal and ventral lesions of the rat spinal cord. Experimental neurology 176:143-153.

Scolding NJ, Rayner PJ, Sussman J, Shaw C, Compston DA (1995) A proliferative adult human oligodendrocyte progenitor. Neuroreport 6:441-445. 
Selbach M, Schwanhausser B, Thierfelder N, Fang Z, Khanin R, Rajewsky N (2008) Widespread changes in protein synthesis induced by microRNAs. Nature 455:58-63.

Semple BD, Blomgren K, Gimlin K, Ferriero DM, Noble-Haeusslein LJ (2013) Brain development in rodents and humans: Identifying benchmarks of maturation and vulnerability to injury across species. Progress in neurobiology 106-107:116.

Shin S, Sun Y, Liu Y, Khaner H, Svant S, Cai J, Xu QX, Davidson BP, Stice SL, Smith AK, Goldman SA, Reubinoff BE, Zhan M, Rao MS, Chesnut JD (2007) Whole genome analysis of human neural stem cells derived from embryonic stem cells and stem and progenitor cells isolated from fetal tissue. Stem cells (Dayton, Ohio) 25:1298-1306.

Sholomenko GN, Steeves JD (1987) Effects of selective spinal cord lesions on hind limb locomotion in birds. Experimental neurology 95:403-418.

Silver J (2009) CNS Regeneration: Only on One Condition. Current biology : CB 19:R444-R446. 
Sim FJ, Lang JK, Waldau B, Roy NS, Schwartz TE, Pilcher WH, Chandross KJ, Natesan S, Merrill JE, Goldman SA (2006) Complementary patterns of gene expression by human oligodendrocyte progenitors and their environment predict determinants of progenitor maintenance and differentiation. Annals of neurology 59:763-779.

Sim FJ, McClain CR, Schanz SJ, Protack TL, Windrem MS, Goldman SA (2011) CD140a identifies a population of highly myelinogenic, migration-competent and efficiently engrafting human oligodendrocyte progenitor cells. Nature biotechnology 29:934-941.

Sotnikov OS (2003) [Unmyelinated nerve fibers]. Morfologiia (Saint Petersburg, Russia) 123:88-96.

Sroga JM, Jones TB, Kigerl KA, McGaughy VM, Popovich PG (2003) Rats and mice exhibit distinct inflammatory reactions after spinal cord injury. The Journal of comparative neurology 462:223-240.

Steelman AJ, Thompson JP, Li J (2012) Demyelination and remyelination in anatomically distinct regions of the corpus callosum following cuprizone intoxication. Neuroscience research 72:32-42. 
Steeves JD, Jordan LM (1980) Localization of a descending pathway in the spinal cord which is necessary for controlled treadmill locomotion. Neuroscience letters 20:283-288.

Takeshita F, Patrawala L, Osaki M, Takahashi RU, Yamamoto Y, Kosaka N, Kawamata M, Kelnar K, Bader AG, Brown D, Ochiya T (2010) Systemic delivery of synthetic microRNA-16 inhibits the growth of metastatic prostate tumors via downregulation of multiple cell-cycle genes. Molecular therapy : the journal of the American Society of Gene Therapy 18:181-187.

Talbott JF, Cao Q, Bertram J, Nkansah M, Benton RL, Lavik E, Whittemore SR (2007) CNTF promotes the survival and differentiation of adult spinal cordderived oligodendrocyte precursor cells in vitro but fails to promote remyelination in vivo. Experimental neurology 204:485-489.

Talbott JF, Loy DN, Liu Y, Qiu MS, Bunge MB, Rao MS, Whittemore SR (2005) Endogenous Nkx2.2+/Olig2+ oligodendrocyte precursor cells fail to remyelinate the demyelinated adult rat spinal cord in the absence of astrocytes. Experimental neurology 192:11-24.

Tasaki I (1939) The electro-saltatory transmission of the nerve impulse and the effect of narcosis upon the nerve fiber Am J Physiol 127:211-227. 
Taylor DD, Gercel-Taylor C (2008) MicroRNA signatures of tumor-derived exosomes as diagnostic biomarkers of ovarian cancer. Gynecologic oncology 110:13-21.

Temple S, Raff MC (1985) Differentiation of a bipotential glial progenitor cell in a single cell microculture. Nature 313:223-225.

Tetzlaff W, Okon EB, Karimi-Abdolrezaee S, Hill CE, Sparling JS, Plemel JR, Plunet WT, Tsai EC, Baptiste D, Smithson LJ, Kawaja MD, Fehlings MG, Kwon BK (2011) A systematic review of cellular transplantation therapies for spinal cord injury. Journal of neurotrauma 28:1611-1682.

Timsit S, Martinez S, Allinquant B, Peyron F, Puelles L, Zalc B (1995) Oligodendrocytes originate in a restricted zone of the embryonic ventral neural tube defined by DM-20 mRNA expression. The Journal of neuroscience : the official journal of the Society for Neuroscience 15:1012-1024.

Tomasch J (1961) Dietary factors and nerve fibre myelination. Journal of anatomy 95:180-190.

Torkildsen O, Brunborg LA, Myhr KM, Bo L (2008) The cuprizone model for demyelination. Acta neurologica Scandinavica Supplementum 188:72-76. 
Totoiu MO, Keirstead HS (2005) Spinal cord injury is accompanied by chronic progressive demyelination. The Journal of comparative neurology 486:373-383. van der Knaap MS, Pronk JC, Scheper GC (2006) Vanishing white matter disease. The Lancet Neurology 5:413-423.

Vasudevan S, Tong Y, Steitz JA (2007) Switching from repression to activation: microRNAs can up-regulate translation. Science (New York, NY) 318:1931-1934.

Vilensky JA, Moore AM, Eidelberg E, Walden JG (1992) Recovery of Locomotion in Monkeys With Spinal Cord Lesions. Journal of motor behavior 24:288-296.

Virchow R (1854) Uber das ausgebreitete Vorkommen einer dem Nervenmark analogen Substanz in den tierischen Geweben. 6:562-572.

Vogel US, Reynolds R, Thompson RJ, Wilkin GP (1988) Expression of the 2',3'cyclic nucleotide 3'-phosphohydrolase gene and immunoreactive protein in oligodendrocytes as revealed by in situ hybridization and immunofluorescence. Glia 1:184-190.

Wake H, Lee PR, Fields RD (2011) Control of local protein synthesis and initial events in myelination by action potentials. Science (New York, NY) 333:16471651. 
Wang Q, Chow J, Hong J, Smith AF, Moreno C, Seaby P, Vrana P, Miri K, Tak J, Chung ED, Mastromonaco G, Caniggia I, Varmuza S (2011) Recent acquisition of imprinting at the rodent Sfmbt2 locus correlates with insertion of a large block of miRNAs. BMC genomics 12:204.

Wang S, Bates J, Li X, Schanz S, Chandler-Militello D, Levine C, Maherali N, Studer L, Hochedlinger K, Windrem M, Goldman SA (2013) Human iPSC-derived oligodendrocyte progenitor cells can myelinate and rescue a mouse model of congenital hypomyelination. Cell stem cell 12:252-264.

Wang Y, Blelloch R (2009) Cell cycle regulation by MicroRNAs in embryonic stem cells. Cancer research 69:4093-4096.

Watson JV, Chambers SH, Smith PJ (1987) A pragmatic approach to the analysis of DNA histograms with a definable G1 peak. Cytometry 8:1-8.

Waxman SG (1989) Demyelination in spinal cord injury. Journal of the neurological sciences 91:1-14.

Wegner M (2008) A matter of identity: transcriptional control in oligodendrocytes. Journal of molecular neuroscience : MN 35:3-12. 
Wilkins A, Chandran S, Compston A (2001) A role for oligodendrocyte-derived IGF-1 in trophic support of cortical neurons. Glia 36:48-57.

Wilkins A, Majed H, Layfield R, Compston A, Chandran S (2003)

Oligodendrocytes promote neuronal survival and axonal length by distinct intracellular mechanisms: a novel role for oligodendrocyte-derived glial cell linederived neurotrophic factor. The Journal of neuroscience : the official journal of the Society for Neuroscience 23:4967-4974.

Wilson HC, Scolding NJ, Raine CS (2006) Co-expression of PDGF alpha receptor and NG2 by oligodendrocyte precursors in human CNS and multiple sclerosis lesions. Journal of neuroimmunology 176:162-173.

Windrem MS, Nunes MC, Rashbaum WK, Schwartz TH, Goodman RA, McKhann G, 2nd, Roy NS, Goldman SA (2004) Fetal and adult human oligodendrocyte progenitor cell isolates myelinate the congenitally dysmyelinated brain. Nature medicine 10:93-97.

Windrem MS, Schanz SJ, Guo M, Tian GF, Washco V, Stanwood N, Rasband M, Roy NS, Nedergaard M, Havton LA, Wang S, Goldman SA (2008) Neonatal chimerization with human glial progenitor cells can both remyelinate and rescue the otherwise lethally hypomyelinated shiverer mouse. Cell stem cell 2:553-565. 
Wu CY, Lu J, Cao Q, Guo CH, Gao Q, Ling EA (2006) Expression of 2',3'-cyclic nucleotide 3'-phosphodiesterase in the amoeboid microglial cells in the developing rat brain. Neuroscience 142:333-341.

Xiao L, Hu C, Yang W, Guo D, Li C, Shen W, Liu X, Aijun H, Dan W, He C (2013) NMDA receptor couples Rac1-GEF Tiam1 to direct oligodendrocyte precursor cell migration. Glia 61:2078-2099.

Yajima K, Suzuki K (1979) Demyelination and remyelination in the rat central nervous system following ethidium bromide injection. Laboratory investigation; a journal of technical methods and pathology 41:385-392.

Yamanaka S, Takahashi K (2006) [Induction of pluripotent stem cells from mouse fibroblast cultures]. Tanpakushitsu kakusan koso Protein, nucleic acid, enzyme $51: 2346-2351$.

Ye P, Bagnell R, D'Ercole AJ (2003) Mouse NG2+ oligodendrocyte precursors express mRNA for proteolipid protein but not its DM-20 variant: a study of laser microdissection-captured NG2+ cells. The Journal of neuroscience : the official journal of the Society for Neuroscience 23:4401-4405. 
Young KM, Psachoulia K, Tripathi RB, Dunn SJ, Cossell L, Attwell D, Tohyama K, Richardson WD (2013) Oligodendrocyte dynamics in the healthy adult CNS: evidence for myelin remodeling. Neuron 77:873-885.

Yuan X, Chittajallu R, Belachew S, Anderson S, McBain CJ, Gallo V (2002) Expression of the green fluorescent protein in the oligodendrocyte lineage: a transgenic mouse for developmental and physiological studies. Journal of neuroscience research 70:529-545.

Zhang YP, Burke DA, Shields LB, Chekmenev SY, Dincman T, Zhang Y, Zheng Y, Smith RR, Benton RL, DeVries WH, Hu X, Magnuson DS, Whittemore SR, Shields CB (2008) Spinal cord contusion based on precise vertebral stabilization and tissue displacement measured by combined assessment to discriminate small functional differences. Journal of neurotrauma 25:1227-1240.

Zhao X, He X, Han X, Yu Y, Ye F, Chen Y, Hoang T, Xu X, Mi QS, Xin M, Wang F, Appel B, Lu QR (2010) MicroRNA-mediated control of oligodendrocyte differentiation. Neuron 65:612-626. 


\section{CURRICULUM VITAE}

NAME: Nicholas John Kuypers

ADDRESS: $\quad 511$ South Floyd

Medical Dental Research Building, room 622

University of Louisville School of Medicine

Louisville, Kentucky 40292

DOB: $\quad$ Chicago, lllinois - April $24^{\text {th }}, 1980$

EDUCATION

\& TRAINING: $\quad$ B.A., Jazz Studies

University of New Orleans

1998-2001

B.A., Psychology (major)

B.S., Physiology (minor)

Southern Illinois University at Carbondale

2005-2008

M.S., Anatomical Sciences and Neurobiology

PhD., Anatomical Sciences and Neurobiology

University of Louisville School of Medicine

2008-2014

AWARDS: $\quad$ Student Spotlight, University of Louisville School of

Interdisciplinary and Graduate Studies (2012)

Michael Tanner award for excellence in graduate research, Research!Louisville (2012)

Graduate poster competition (2nd place), Neuroscience Day, University of Louisville (2012)

Poster competition (1st place), Undergraduate Research

Forum, Southern Illinois University (2008) 
Gordon F. Pitz award (outstanding honors thesis in

psychology), Southern Illinois University (2008)

B.A. in psychology awarded with honors (2008)

Research Enriched Academic Challenge (REACH) award, Southern Illinois University (2007)

Louis Armstrong Scholarship (4-year jazz performance scholarship), University of New Orleans (1998)

Outstanding Jazz Performance Award, Prairie State College PROFESSIONAL Jazz Festival (1997)

SOCIETIES: National Neurotrauma Society (2006-present)

Society for Neuroscience (2010-present)

PUBLICATIONS: Kuypers NJ, James KT, Enzmann GU, Magnuson DS, Whittemore SR (2013). Functional consequences of ethidium bromide demyelination of the mouse ventral spinal cord. Exp Neurol. 247: 615-622

Zhu Q, Whittemore SR, Devries WH, Zhao X, Kuypers NJ, Qiu M (2011). Dorsally-derived oligodendrocytes in the spinal cord contribute to axonal myelination during development and remyelination following focal demyelination. Glia. 59:1612-21

Kuypers NJ, Hoane MR (2010). Pyridoxine administration improves behavioral and anatomical outcome after unilateral contusion injury in the rat. J Neurotrauma. 27:1275-82

Mishra PK, Kuypers NJ, Singh SR, Diaz N, Chavali V, Tyagi SC (2013) Cardiac stem cell niche, MMP9, and culture and differentiation of embryonic stem cells. In Kursad Turksen (Ed): Stem Cells and Niche, Methods and Protocols, Springer Publication, In Press.

NATIONAL MEETING

PRESENTATIONS: Society for Neuroscience Nanosymposium (San Diego, CA), "Dynamically regulated oligodendrocyte precursor cell (OPC) microRNAs (miRNAs) during cuprizone induced demyelination and Remyelination." (2013) 
REFEREED

JOURNALS: $\quad$ Experimental Neurology

Neuroscience Letters

INVITED

PRESENTATIONS: Institute for Cellular Therapeutics, University of Louisville School of Medicine, "Role of miRNAs in oligodendrocyte remyelination." (2012) 